FOOD SECURITY IN PRACTICE

\title{
Measuring Food Security Using Household Expenditure Surveys
}

Lisa C. Smith and Ali Subandoro 
Copyright ( 2007 International Food Policy Research Institute. All rights reserved. Sections of this material may be reproduced for personal and not-for-profit use without the express written permission of, but with acknowledgment

to, IFPRI. To reproduce the material contained herein for profit or commercial use requires express written permission.

To obtain permission, contact the Communications Division <ifpri-copyright@cgiarorg>.

International Food Policy Research Institute

2033 K Street, N.W.

Washington, D.C. 20006-1002

U.S.A.

Telephone +1-202-862-5600

www.ifpri.org

How to cite this book: Smith, Lisa C., and Ali Subandoro. 2007. Measuring Food Security Using

Household Expenditure Surveys. Food Security in Practice technical guide series. Washington, D.C.:

International Food Policy Research Institute.

DOI: $10.2499 / 0896297675$

\section{Library of Congress Cataloging-in-Publication Data}

Smith, Lisa C.

Measuring food security using household expenditure surveys /

by Lisa C. Smith, Ali Subandoro.

p. $\quad \mathrm{cm}$.

Includes bibliographical references.

ISBN-13: 978-0-89629-767-8 (pbk. : alk. paper)

ISBN-10: 0-89629-767-5 (pbk.: alk. paper)

1. Food supply. 2. Food consumption. 3. Diet. 4. Household

surveys. I. Subandoro, Ali. II. Title.

HD9000.5.S62 2007

$338.1^{\prime} 9-\mathrm{dc} 22$ 


\section{Contents}

List of Tables, Figures, and Boxes

Foreword

Acknowledgments

1. Introduction

2. HES Indicators of Food Security

Measuring Indicators of Diet Quantity, Diet Quality,

and Economic Vulnerability . . . . . . . . . . . . . . . . . . . .5

Measurement Issues to Keep in Mind . . . . . . . . . . . .8

3. Collecting the Food Data from Households

.13

Strategies for Collecting Data on Quantities

of Foods Acquired ......................... 13

Other Choices to Be Made in Questionnaire Design . . . . . . 23

Drafting the Questionnaire . . . . . . . . . . . . . . . . 29

Conducting the Interviews: Some Practical Points. ...... 33

Model Household Questionnaire ...

4. Gathering Data for Calculating Metric Weights of Foods and Their Energy Content ..............44

Metric Weights of Foods Measured in Nonstandard Units . . 47

Metric Weights of Foods Measured in Volumetric Units,

in Linear Dimensions, and Using Food Models . . . . . . . . .49

Proportionate Weights of Ingredients in Prepared Dishes Consumed Outside the Home. . .

Metric Food Prices ....................... 50

Primary Equivalents of Processed Foods. .............54

Energy Content of Foods and Edible Portions............ 54

5. Processing and Cleaning the Data $\ldots \ldots \ldots \ldots \ldots 7$

Assembling the Raw Data Collected from Households and Initial Data Cleaning ..................... 57

Processing and Cleaning Metric Conversion Factors,

Metric Prices, and Proportionate Weights of Ingredients

in Prepared Dishes. . . . . . . . . . . . . . . . . . . . . . 59

Calculating the Number of Household Members, Adult Equivalents, and Energy Requirements .............64

Calculating and Cleaning Daily Household Metric

Food Quantities ........................66

Calculating Daily Household Total Food Expenditures . . . 69

Calculating and Cleaning Daily Household

Energy Acquisition. ....................... 70

6. Calculating the Indicators $\ldots \ldots \ldots \ldots \ldots \ldots \ldots 75$

Daily Food Energy Consumption per Capita . . . . . . . . . . . 75

Percentage of Households or People That Are Food Energy Deficient. . . . . . . . . . . . . . . . . . . . . . . 75

Diet Diversity . . . . . . . . . . . . . . . . . . 78

Percentage of Food Energy from Staples. . . . . . . . . . . . . . 78

Daily Quantities of Specific Foods Consumed per Capita . . 79

Percentage of Expenditures on Food. ................ . 79 
7. Using the Indicators for Food Security Analysis.....81

Where Are the Food-Insecure? An Example from Senegal . . 81

What Is the Nature of the Food Insecurity Problem?

An Example from Lao PDR .......................87

How Does Food Insecurity Change over Time?

An Example from Bangladesh.

What Are the Causes of Food Insecurity? An Example from Uganda ........................ 91

Which Are the Most Important Foods in the Diets

of Different Sociodemographic Groups? An Example

from Tanzania............................94

Appendix 1: Food Groups with a Listing of the Most

Common Foods ..........................97

Appendix 2: Local Units of Measure Used in 20 National

Household Expenditure Surveys .................999

Appendix 3: Cup Weights of Commonly Consumed

Foods

Appendix 4: Specific Gravities of Commonly Consumed

Beverages and Other Liquid Foods

Appendix 5: Divergence of Market Prices from Unit Values for 12 Foods, Papua

New Guinea Household Survey 1995-96:

Comparison of Gibson and Rozelle

and AFINS Project Results
Appendix 6: Edible Percentages of Commonly

Consumed Foods.

Appendix 7: STATA 8.0 Program for Calculating the Number of Household Adult Equivalents and Household Energy Requirements

Appendix 8: Recommended Daily Caloric Intakes from the 2001 Expert Consultation, by Age, Sex, and Activity Level.

Appendix 9: STATA 8.0 Program for Estimating Metric Food Quantities to Replace Missing Values. . 138

Appendix 10: STATA 8.0 Program for Calculating and Cleaning Household Daily Energy Acquisition . . 140

Appendix 11: The Price-per-Calorie Method of Estimating the Energy Content of Foods Consumed Outside the Home

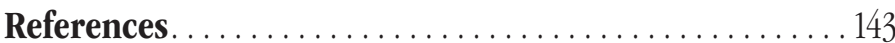

About the Authors 


\section{Tables, Figures, and Boxes}

\section{TABLES}

1. National household expenditure surveys analyzed as part of the AFINS project. . ...................

2. Indicators of food security and their household-level measures .............................6

3. Choosing a method for collecting data on foods acquired for in-home consumption: Cost considerations . . . . . . . . 15

4. Choosing a method for collecting data on foods acquired for in-home consumption: Accuracy considerations. . . . . . . . 16

5. Summary of methods of data collection suitable to different types of foods. ..................... 17

6. Recall and reference periods used for food data collection in AFINS project surveys using the interview approach . . . . . .28

7. Recommended daily caloric intake, by age group ........65

8. Food security indicators calculated from household expenditure survey data collected in 20 developing countries . . 76

9. Guidelines for interpreting indicator levels . . . . . . . . . . 82

10. Food security indicators for administrative regions and rural versus urban areas of Senegal, $2001 \ldots \ldots \ldots . .84$

11. Diet quantity and quality food security indicators for

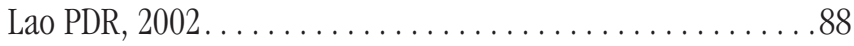

12. Food security indicators for Uganda, by total expenditure quintile and education and gender of household head, 1999 . .92

13. Consumption of food staples in Tanzania (grams per capita per day)

\section{FIGURES}

1. Distributions of household calorie acquisition per capita for eight AFINS project countries. . . . . . . . . . . . . . . 72

2. Prevalences of food energy deficiency for the administrative regions of Senegal, 2001

3. Diet quantity indicators for Bangladesh, by month, January-December 2000 . . . . . . . . . . . . . . . . . . 990

4. Diet quality indicators for Bangladesh, by month, January-December $2000 \ldots \ldots \ldots \ldots \ldots \ldots \ldots \ldots \ldots \ldots$

5. Daily food energy consumption and diet diversity in Uganda, by total expenditure quintile and adult education, 1999 .

6. Consumption of maize, rice, sorghum, and cassava in Tanzania, by income quintile, $2000 \ldots \ldots \ldots \ldots . \ldots 96$

\section{BOXES}

1. Methods of Collecting Data on Food Quantities ............. 14

2. Recommended Method for Collecting Data to Calculate Quantities of Foods in Prepared Dishes ..........22

3. Basic Principles for Constructing a Food List . . . . . . . . . . 25

4. Types of Foods Containing Inedible Parts. . . . . . . . . . . 55

5. Data Processing and Cleaning Steps . ............... 57

6. Sample Layout of Data File with the Raw Data Collected

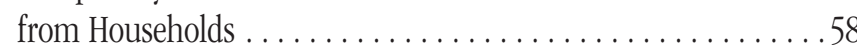

7. Sample Layout of Data File with Metric Conversion Factors Collected in Markets .........................60 60 
8. Sample Layout of Data File with Price Data Collected in Markets

9. Sample Layout of Data File with Metric Prices of Prepared Dishes and Proportionate Weights of Their Ingredients . . . . . 63

10. Where Are the Food-Insecure? Deciding on the Level of Disaggregation............................ 83

11. Statistical Significance and Practical Importance ......... 86

vi Measuring Food Security Using Household Expenditure Surveys 


\section{Foreword}

F

ood is one of the most basic needs for human survival.

Access to it is a basic human right. Moreover, the pursuit of the Millennium Development Goal to cut hunger requires a sound understanding of the related food security issues. For these reasons, accurate measurement of the food security status of populations — or their ability to gain access to sufficient high-quality food to enable them to live an active, healthy life-is imperative to all international development efforts. It is necessary for effectively targeting food-insecure populations, researching and planning appropriate interventions, and monitoring progress.

As past efforts have shown, accurately estimating the amount of food people eat is costly in terms of time and money, and such measurements have thus been carried out mostly in small populations. Where measurement has been extended to large populations, such as entire countries, it has been necessary to rely on less accurate, indirect techniques based on the availability of food at the national level. This technical guide presents a new avenue for measuring food security, for both small and large populations, based on the data collected as part of household expenditure surveys on the quantities of food acquired by households. It shows how these data can be used to measure a variety of food security indicators, including the prevalence of food energy deficiency and indicators of dietary quality and economic vulnerability to food insecurity.

In keeping with the approach of IFPRI's Food Security in Practice series for practitioners, the manual guides readers step by step through the process of assessing the food security status of a population. It begins by offering guidance on choosing an appropriate strategy for calculating quantities of foods acquired by households, given time constraints, financial constraints, and the nature of the population's diet. The guide then leads the practitioner through the steps of collecting the data, processing and cleaning the data, and calculating the indicators. It concludes by illustrating how to conduct some basic food security analyses.

I hope that this guide will assist practitioners in increasing the accuracy of the measurement of food insecurity for a greater number of populations, including those at the country level. Greater accuracy at the country level will provide the necessary foundations for overcoming food insecurity globally.

Joachim von Braun

Director General, IFPRI 


\section{Acknowledgments}

unding for this manual has been generously provided by

the Department for International Development of the United Kingdom (DfID). Research for the manual was undertaken under the auspices of the project Improving the Empirical Basis for Assessing Food Insecurity in Developing Countries (the AFINS project), which is funded by a consortium of donors, including the Australian Agency for International Development, the Canadian International Development Agency, DfID, the United States Agency for International Development's Presidential Initiative to End Hunger in Africa, the U.S. Department of Agriculture, and the World Bank, as well as the group of donors that provide unrestricted funding to IFPRI.

The following government statistical services administered the AFINS project surveys and compiled the datasets used for the research:

- Institut de Statistiques et d'Études Économiques du Burundi,

- Central Statistical Authority of Ethiopia,

- Ghana Statistical Service,

- Direction Nationale de la Statistique, République de Guinée,

- Central Bureau of Statistics of Kenya,

- National Statistical Office of Malawi,

- Instituto Nacional de Estatistíca de Mozambique,

- Direction de la Statistique de Rwanda,

- Direction de la Prévision et de la Statistique de Senegal,
- National Bureau of Statistics of Tanzania

- Uganda Bureau of Statistics,

- Central Statistical Office of Zambia,

- Bangladesh Bureau of Statistics,

- National Sample Survey Organization of India,

- Central Bureau of Statistics of Indonesia,

- National Statistical Center of Lao, People's Democratic Republic,

- Department of Statistics of Malaysia,

- Pakistan Federal Bureau of Statistics, and

- Department of Census and Statistics of Sri Lanka.

The World Bank administered the survey from Papua New Guinea. We thank the staff members of these organizations who helped to answer our questions and find the additional data we needed to undertake the analysis. We also thank the World Bank for facilitating access to some of the datasets. Dede Aduayom, Joshua Ariga, Elizabeth Byron, Smita Ghosh, Aida Ndaiye, Ellen Payongayong, Arif Rashid, Ibrahima Wane, and Doris Wiesmann assisted in assembling and analyzing the datasets.

We thank Lawrence Haddad for his role in instigating the AFINS project research program and Marie Ruel and Joachim von Braun for their continued support. We also gratefully acknowledge the comments of three anonymous reviewers and of Tom Spangler of TANGO International. 


\section{Measuring Food Security \\ Using Household Expenditure Surveys}




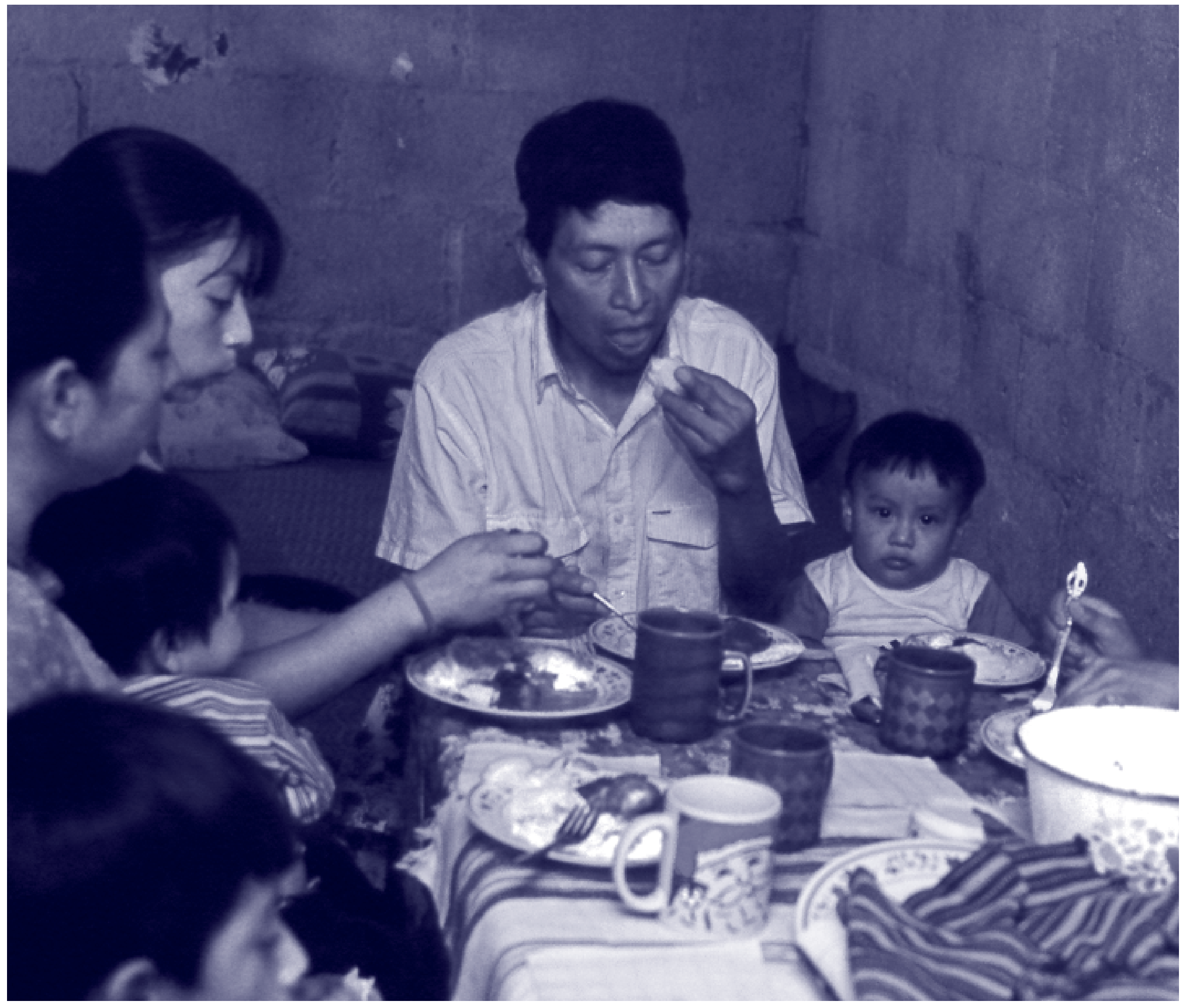




\section{Introduction}

$\mathbf{F}$ ood insecurity continues to be a major development problem across the globe, undermining people's health, productivity, and often their very survival. Efforts to overcome the development challenges posed by food insecurity necessarily begin with accurate measurement of key indicators at the household level. This is due to the fact that identification of household behaviors relating to food access serves as a critical building block for the development of policies and programs for helping vulnerable populations, the effective targeting of assistance, and the evaluation of impact.

This manual is a guide for measuring food security using the food data collected as part of household expenditure surveys (HESs). While the main focus of these surveys has been on measuring households' total monetary expenditures as a proxy for income, the surveys typically collect data on a variety of other aspects of people's well-being as well, such as education, housing, and health. With respect specifically to food, data are collected on all foods acquired by households, including their food purchases, foods consumed from their own farms or gardens, and foods received in kind. Traditionally, data collection has been limited to the monetary value of these foods. However, as this manual demonstrates, accurate measurement of food security requires that data also be collected that allow estimation of the quantities of foods acquired by households. Estimated quantities, expressed in metric units, then serve as the basis for calculating a number of indicators of food security, including diet quantity, diet quality, and economic vulnerability to food insecurity. These indicators include
- dietary energy availability per capita,

- percentage of people who are food energy deficient,

- diet diversity,

- percentage of dietary energy derived from staples,

- quantities of individual foods consumed per capita, and

- percentage of household expenditures devoted to food.

The primary objective of the manual is to inform HES data collectors and users how to collect the food data and use it to measure these indicators at the least possible additional cost.

The manual has been written as a tool for a variety of development practitioners and researchers, including (1) technicians in governments and international development agencies comparing food security within and across countries so that assistance can be targeted most effectively and changes in food insecurity over time can be monitored, (2) project managers planning and implementing Monitoring and Evaluation systems, and (3) researchers investigating the causes of food insecurity and the impact of specific policies and programs.

With respect specifically to national estimates of food security, the percentage of countries' populations that are food energy deficient is the indicator that is currently being used to monitor countries progress in meeting international goals such as the Millennium Development Goal of halving the proportion of people who suffer from hunger by 2015. Low-cost, yet imprecise, food balance sheet-based 
estimates of the indicator published by the United Nations Food and Agriculture Organization (FAO) are the conventional basis for international comparisons. These estimates are based on the amounts of foods available at a national level in countries rather than on data collected directly from households. On the other hand food consumption data, while collected directly from households using very precise measurement techniques, are too costly to collect on a national basis for most countries. HES-based estimates are a less costly third option. This is because data collection focuses on foods acquired rather than actual prepared dishes consumed, which makes it far less complicated to measure food quantities. While measurement is less precise than that of food consumption surveys, it is reasonably accurate, yielding roughly the same estimates of food energy deficiency for population groups. As more and more countries begin to collect the appropriate data in their national HESs, such surveys are becoming a viable option for monitoring national and global food insecurity (Smith, Alderman, and Aduayom 2006).

The manual draws on the data processing and analysis experience of HESs conducted in 12 countries of Sub-Saharan Africa and 8 of Asia since the early 1990s as part of the project called Improving the Empirical Basis for Assessing Food Insecurity in Developing Countries (the AFINS project), a joint undertaking of the International Food Policy Research Institute and the World Bank. Information on these surveys is given in Table 1. More details can be found in Smith and Subandoro (2005) and Smith, Alderman, and
Aduayom (2006). The manual also draws on the long experience of food consumption surveys to expand the set of options available for measuring metric food quantities by introducing methods with greater precision than those traditionally used in HESs. It helps users choose the most accurate measurement technique for a specific population group that fits within time and monetary constraints.

Note that the manual does not address the important issues of sampling design, integrating survey modules into a complete questionnaire, pretesting questionnaires, and interviewer training. Detailed discussions of these and other general issues concerning household surveys can be found in Designing Household Survey Questionnaires for Developing Countries: Lessons from 15 Years of the Living Standards Measurement Study (Grosh and Glewwe 2000). It also does not give an in-depth treatment of indicator choice.

The next chapter introduces the indicators of food security and insecurity that can be measured using HESs. It lays out a number of measurement issues to keep in mind. Chapter 3 goes on to discuss the process of collecting the food data from households, including questionnaire design and drafting. Chapter 4 focuses on gathering the data needed to translate the food data collected from households into metric quantities and their energy content. Chapter 5 takes the reader through the stages of data processing, including data cleaning. Chapter 6 provides instruction on how to calculate indicators of food security. Finally, Chapter 7 shows how to conduct basic food security analysis using the calculated indicators. 
Table 1. National household expenditure surveys analyzed as part of the AFINS project

\begin{tabular}{|c|c|c|c|}
\hline Country & $\begin{array}{l}\text { Year of data } \\
\text { collection }\end{array}$ & Name of survey & Data collection agency \\
\hline \multicolumn{4}{|l|}{ Sub-Saharan Africa } \\
\hline Burundi & 1998 & $\begin{array}{l}\text { Enquête Prioritaire 1998-Etude nationale sur les conditions de vie } \\
\text { des populations }\end{array}$ & $\begin{array}{l}\text { Institut de Statistiques et d'Etudes } \\
\text { Economiques du Burundi }\end{array}$ \\
\hline Ethiopia & 1999 & Household Income, Consumption and Expenditure Survey 1999/2000 & Central Statistical Authority of Ethiopia \\
\hline Ghana & 1998 & Ghana Living Standards Survey 4 & Ghana Statistical Service \\
\hline Guinea & 1994 & $\begin{array}{l}\text { Enquête intégrale sur les conditions de vie des ménages guinéens } \\
\text { avec module budget et consommation }\end{array}$ & Direction Nationale de la Statistique \\
\hline Kenya & 1997 & Welfare Monitoring Survey III & Central Bureau of Statistics \\
\hline Malawi & 1997 & Integrated Household Survey 1997/98 & National Statistical Office \\
\hline Mozambique & 1996 & $\begin{array}{l}\text { Mozambique inqérito nacional aos agregados familiares sobre as } \\
\text { condições de vida }\end{array}$ & Instituto Nacional de Estatística \\
\hline Rwanda & 2000 & $\begin{array}{l}\text { Enquête intégrale sur les conditions de vie des ménages au } \\
\text { Rwanda }\end{array}$ & $\begin{array}{l}\text { Direction de la Statistique du Ministère des } \\
\text { Finances et de la Planification Economique }\end{array}$ \\
\hline Senegal & 2001 & Enquête Sénégalaise aupres des ménages II & Direction de la Prévision et de la Statistique \\
\hline Tanzania & 2000 & Tanzanian Household Budget Survey & National Bureau of Statistics of Tanzania \\
\hline Uganda & 1999 & Uganda National Household Survey 1999/2000 & Uganda Bureau of Statistics \\
\hline Zambia & 1996 & Zambia Living Conditions Monitoring Survey I (1996) & Central Statistical Office \\
\hline \multicolumn{4}{|l|}{ Asia } \\
\hline Bangladesh & 2000 & Household Income and Expenditure Survey 2000 & Bangladesh Bureau of Statistics \\
\hline India & 1999 & National Sample Survey 55th Round Socio-Economic Survey & National Sample Survey Organization \\
\hline Indonesia & 1999 & National Socio-Economic Survey 1999 & Central Bureau of Statistics \\
\hline Lao PDR & 2002 & Lao PDR Expenditure and Consumption Survey III 2002/3 & National Statistical Center \\
\hline Malaysia & 1998 & Malaysia Household Expenditure Survey 1998/99 & Department of Statistics \\
\hline Pakistan & 1998 & Pakistan Integrated Household Survey 1998/99 & Pakistan Federal Bureau of Statistics \\
\hline Papua New Guinea & 1996 & Papua New Guinea Household Survey 1996 & World Bank \\
\hline Sri Lanka & 1999 & Sri Lanka Integrated Survey 1999/2000 & Department of Census and Statistics \\
\hline
\end{tabular}

Source: Smith and Subandoro (2005) and Smith, Alderman, and Aduayom (2006). 


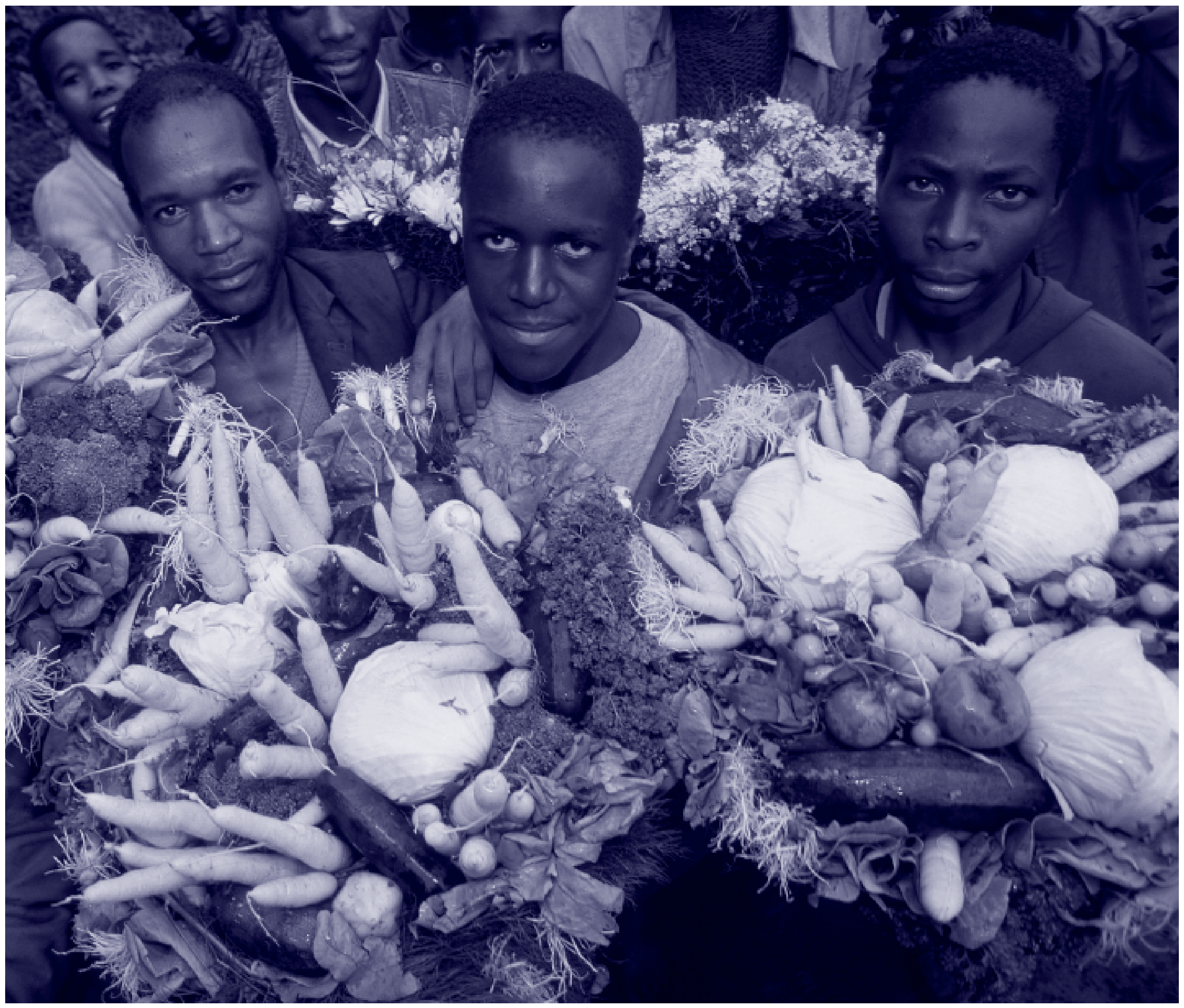




\section{HES Indicators of Food Security}

$\mathrm{T}$

his chapter begins by defining and discussing the meanings of some key indicators of food security that can be measured using HES data. It then lays out some measurement issues it is important to be aware of when using the data for food security analysis.

\section{MEASURING INDICATORS OF DIET QUANTITY, DIET QUALITY, AND ECONOMIC VULNERABILITY}

The indicators of food security focused on in this guide and that can be measured using HES data are listed in Table 2. Each addresses some aspect of the following definition of food security, adopted at the 1996 World Food Summit: "Food security ... [is achieved] when all people, at all times, have physical and economic access to sufficient, safe and nutritious food to meet their dietary needs and food preferences for an active and healthy life" (FAO 1996b). The left-hand column of the table gives the indicator for a population group. The righthand column gives the associated measure at the household level.

The first two indicators are of diet quantity, the amount of food eaten by people. Food energy consumption is measured at the household level as the total amount of energy in the food acquired by the household over the survey reference period for food data collection (the total time for which data are recorded). Calculation of this measure starts with the food data collected from households, either (1) expenditures on each food or (2) quantities acquired of the foods, which are often reported in nonstandard or "local" units of measure, such as bunches or cans. The data are then converted to metric quantities (for example, kilograms) by dividing reported expenditures by metric prices or multiplying reported quantities in local units of measure by a metric weight. The energy contents of the edible portion of all foods acquired are then summed and divided by the number of days in the reference period and the number of household members to arrive at the total energy acquired per household member. Note that although the data collected from households represent foods acquired, the mean of this measure across a randomly selected sample of households is felt to be a reasonably good estimate of mean food energy consumed by a population (see next section).

The second diet quantity indicator is the percentage of households in a population group who do not consume sufficient dietary energy. It is measured by determining whether a household acquires sufficient food over the reference period to meet the dietary energy requirements of all of its members. If the estimated total energy in the food that the household acquires daily is lower than the sum of its members' daily requirements, the household is classified as food energy deficient. The requirements employed are those for basal metabolic function (a state of complete rest) and light activity, such as sitting and standing. When the percentage of people, as opposed to households, is measured, each person is assigned the energy deficiency status of her or his household.

The diet quantity indicators are closely related to the notion of access to food by people, a fundamental component of the definition 


\section{Table 2. Indicators of food security and their household-level measures}

\begin{tabular}{l|l} 
Population-level indicator & Household-level measure \\
Diet quantity & $\begin{array}{l}\text { Household daily food energy availability per capita. The energy in the food acquired by a household over } \\
\text { the survey reference period divided by the number of household members and the number of days in the } \\
\text { period. }\end{array}$ \\
\hline Daily food energy consumption per capita & $\begin{array}{l}\text { Whether a household is food energy-deficient. Whether a household acquires insufficient food over the } \\
\text { reference period to meet the energy requirements of all of its members for basal metabolic function and } \\
\text { light activity. (An individual's energy-deficiency situation is defined as that of her or his household.) }\end{array}$ \\
\hline $\begin{array}{l}\text { Percentage of households or people that } \\
\text { are food energy-deficient }\end{array}$ & $\begin{array}{l}\text { Household diet diversity. The number of foods or nutritionally significant food groups acquired by a } \\
\text { household over the reference period. }\end{array}$ \\
\hline Diet quality & $\begin{array}{l}\text { Percentage of food energy available from staples. The percentage of the energy acquired by a household } \\
\text { over the reference period that is derived from staple foods (cereals, roots, and tubers). }\end{array}$ \\
\hline Diet diversity & $\begin{array}{l}\text { Quantities of foods acquired daily per capita. Ouantity of specific foods acquired over the reference period } \\
\text { divided by the number of household members and the number of days in the period. }\end{array}$ \\
\hline Percentage of food energy from staples & \\
\hline $\begin{array}{l}\text { Ouantities of foods consumed daily } \\
\text { per capita }\end{array}$ & $\begin{array}{l}\text { Percentage of expenditures on food. The percentage of total household expenditures devoted to food over } \\
\text { the reference period. }\end{array}$ \\
\hline Current economic vulnerability &
\end{tabular}

of food security. Energy from food is arguably the most important nutrient for survival, physical activity, and health, and households are the units through which people generally access food. The indicators pertain to the amount and sufficiency of energy in the food that is immediately available to households for consumption, which is a clear indication of their ability to access sufficient food.

The next three indicators measure diet quality, which is as important for achieving food security as diet quantity. It is quite possible for a person to meet her or his energy requirement but to be prevented from leading an active, healthy life due to deficiencies of other nutrients, specifically protein and micronutrients such as iron, vitamin A, and iodine (Welch 2004). A number of studies have documented that improved diet quality is associated with improved birth weight and child nutritional status and with reduced mortality (Ruel 2002, 2003). Further, it is increasingly recognized that inadequate diet quality rather than insufficient energy consumption is becoming the main dietary constraint facing poor populations across the globe (Ruel et al. 2003; Graham, Welch, and Bouis 2004). For these reasons, it is critically important that indicators of the nutritional quality of the food people eat be included in any analysis of food security. 
The first diet quality indicator, diet diversity, reflects how varied the foods typically consumed by a household are. Previous research from both developed and developing countries has consistently shown that diet diversity is a good indicator of nutrient adequacy, that is, of a diet that meets requirements for protein, energy, and all essential nutrients (Ruel 2002). Based on the quantity or expenditure data collected from households, diet diversity is measured simply as the number of foods or nutritionally significant food groups from which food is acquired over the survey reference period. Diet diversity indicators based on food groups predict nutrient adequacy better than those based on individual foods (Ruel 2002). Examples of nutritionally significant food groups are cereals; roots, tubers, and plantains; pulses, legumes, nuts, and seeds; vegetables and fruits; meats, fish, and seafood; milk and dairy products; eggs; oils and fats; beverages; and miscellaneous foods. See Appendix 1 for a list of these food groups, along with the most common foods in each group and the forms in which they are available.

While it is possible to compute measures of low diet diversity (or low diet quality) using HES data, there are currently no international recommendations for optimal food or food-group diversity and thus for determining whether a household or individual has a low-quality diet based only on knowledge of which foods are eaten. Ruel (2002), Arimond and Ruel (2004), and Swindale and Ohri-Vachaspati (2004) discuss ways of determining meaningful benchmarks that can be used to compare the adequacy of diet quality of subgroups within a population.

The second diet quality indicator is the percentage of food energy acquired from staples. At the household level it is measured as the percentage of dietary energy available from food staples (for example, rice, maize, and cassava) in the total dietary energy available. A higher value indicates lower diet quality, because energy-dense starchy staples have small amounts of bioavailable protein and micronutrients, leaving those consuming large amounts of them compared to other foods vulnerable to protein and micronutrient deficiencies

A third diet quality indicator is the quantity of individual foods consumed. Information on specific foods is often of interest to policymakers aiming to improve food security in a particular population or region. For instance, knowledge of the amounts consumed of individual foods rich in particular nutrients may serve as the basis for policies aimed at reducing nutrient deficiencies. Similarly, in countries where many households are not consuming sufficient dietary energy, policymakers may be interested in the consumption of energy-dense staple foods. Alternatively, policymakers hoping to stem chronic disease may be interested in the consumption of foods containing particular types of fats.

It is possible to estimate availabilities of protein and micronutrients using a method similar to that used to estimate energy consumption. In the case of micronutrients it is important to be sure to specify that what is being estimated is the availability of the nutrient in the food acquired by a household rather than nutrient intake. This is because the nutrient content of many foods changes depending on the form in which it is consumed. For example, it may be different when a food is in its raw form than when it is in its cooked form. Alternatively, special adjustment factors that take into account changes in nutrient content due to processing and cooking can be used to estimate intakes of a nutrient (Gibson and Ferguson 1999).

The final food security indicator is the percentage of total household expenditures on food, an indicator of current economic 
vulnerability. Households that spend a large proportion of their income on food (greater than 75 percent, say) are vulnerable to food deprivation because, regardless of their current food consumption status, if they were to experience a reduction in income it would likely be accompanied by a reduction in food consumption or the quality of food eaten.

As a group, the indicators featured in this manual cover important aspects of the definition of food security given earlier: access, sufficiency, and quality. It is important to keep in mind, however, that certain aspects of food security cannot be measured using the data collected in HESs as they are currently designed. A complete picture of the state of food security should address temporal shifts in food access — whether households have access to food at all times. Yet the indicators do not address vulnerability to food deprivation in the future. Further, the definition of food security stresses that all people have access to food. Yet the food data collected in HESs indicate the access to food of household units, not individuals within them. It is now well known that intrahousehold food distribution is not always such that all household members receive the food that they need, even if sufficient food is available at the household level (Haddad and Kanbur 1990). Finally, the indicator set does not address issues of food safety and food preferences.

\section{MEASUREMENT ISSUES TO KEEP IN MIND}

As mentioned in the introduction, while HES data can be used to measure the same indicators as food consumption surveys at far less cost, they are believed to give less accurate estimates. The data collected in food consumption surveys measure the amounts of foods actually eaten by households, often broken down by individuals within households, as recalled over the previous 24-hour period. By contrast, data collected in HESs measure the amounts of foods acquired by households, usually over the previous one to two weeks, which invites increased recall error. Yet available studies comparing key indicators of food security calculated at the population group level (daily energy consumption per capita and the percentage of people who are food energy deficient) using food consumption and food acquisition data collected from the same households find little difference between estimates from the two types of data (Smith, Alderman, and Aduayom 2006). Although a definitive determination awaits future research on a variety of populations, this evidence suggests that measures of food security estimated using HESs are reasonably accurate.

Nevertheless, users of HES data should be aware of some key measurement issues that will aid them in understanding the appropriate uses of data for food security analysis and their limitations (see Smith, Alderman, and Aduayom 2006 for more detail).

\section{Food Wasted and Given to Pets and Guests Is Not Accounted For}

The first issue is that, in contrast to food consumption surveys, HESs do not collect data on food wasted and food given to pets and guests. ${ }^{1}$

${ }^{1}$ A rare exception is a survey undertaken in urban Papua New Guinea in 1985-87, in which a roster of people present at each meal was kept (see Gibson and Rozelle 2002), making it possible to adjust estimates of dietary energy consumption for transfers of food between households. 
Thus, for a population group in which these elements of food use are high, estimates of food and dietary energy consumption will be biased upward and vice versa. A study of small population groups in Kenya, the Philippines, and Bangladesh found that for the populations as a whole, estimates of household food energy consumption and household food energy acquisition were roughly equal. However, for the lowestincome groups, energy acquisition was systematically lower than energy consumption, while for the highest-income groups the opposite was true. This means that estimates of the relationship between income and energy consumption using HES data can be biased upward (Bouis and Haddad 1992; Bouis, Haddad, and Kennedy 1992).

\section{The Measurement Techniques in HESs Are Not as Precise as Those Used in Food Consumption Surveys}

The expenditure or quantity data collected from households as part of HESs are translated into metric units using metric prices or metric weights of nonstandard units of measure collected at the level of the community or an even higher geographic area. In food consumption surveys all data used are collected at the household level using more direct techniques, such as actual weighing of foods using scales, use of two- or three-dimensional models of various sizes and shapes for each food, or estimations using volumetric containers. Further, due to the complexities of measuring food consumed outside the home, past surveys have collected only data on the total expenditures of a household on these foods, with no identification of the foods actually eaten or the amounts. This technique leads to various errors in the estimation of total food quantities acquired, household energy availability, and diet diversity, especially for populations for which food eaten out is an important part of the diet. The following chapter presents some measurement options that improve on the techniques typically used in HESs by borrowing those used in food consumption surveys.

\section{Care Needs to Be Taken in Interpreting Household-Level Estimates of Food Energy Availability}

It is important to keep in mind, especially for data processing and cleaning, the fact that the data collected in HESs are on foods acquired rather than consumed and to understand the implications of this difference. Because most foods are perishable and consumed with high frequency and people try to smooth their consumption of food over time, we would expect their acquisitions to match fairly well with consumption, even over a short time period. However, certain foods, such as some grains, are not perishable and can be stored. Thus, over any given time period there will be households that are drawing down stocks acquired before the period in order to meet current consumption needs, while others will be accumulating stocks for consumption after the period. Therefore, the amounts of food acquired and consumed over the same time period will not always be equal. In fact, in the typical HES there will be households with calorie acquisition estimates far below what is needed for human survival. There will also be estimates that are far above what a person could possibly eat in one day. Randomly selected households in a population group are equally likely to be drawing down on food stocks as they are to be accumulating them. Thus, as previous studies have confirmed, the difference at the household level represents "random error," and mean household calorie consumption should theoretically be the same as household calorie availability. 


\section{Population-Group Estimates of Food Energy Deficiency Are Likely to Be Reasonably Accurate, but Further Research Is Needed}

When it comes to estimates of food energy deficiency, this lack of bias does not necessarily apply. The variability in food acquisition data is far higher than that in food consumption data. Estimates of per capita energy availability from food acquisition data can legitimately range from 0 to 12,000 kilocalories per capita or more, far outside the range of appropriate energy consumption for human beings. For this reason, estimates of food energy deficiency can theoretically be different depending on which data source is used. In particular, if the population energy requirement is below the mode of the energy availability distribution, estimates of the prevalence of food energy deficiency are biased upward and vice versa. As mentioned earlier, current evidence suggests that this is not a major issue in most cases. The increased variability of HES food acquisition data would have to be quite large to lead to a meaningful difference in estimates of food energy deficiency. Nevertheless, the existence and magnitude of this bias need to be investigated further.

\section{A Normative Rather than Actual Energy Requirement Is Used in Estimating Food Energy Deficiency}

Actual energy requirements of individuals depend on their age, sex, body size, activity level, and individual physiology, for example, metabolism. When determining the energy needs of a group of individuals, given unknown actual requirements (because of individual variation), the Expert Consultation on Energy and Protein
Requirements (FAO, WHO, and UNU 1985; see also UNU, WHO, and FA0 2004) recommends the use of average energy requirements for people of different sex and age groups, levels of activity, and, for adults, body size, which apply to all individuals globally. In HESs, data are collected on age and sex but none of the other characteristics. Use of the "light" activity level is recommended here as a normative standard applicable to all populations. A person who does not consume enough food to meet the energy requirement for basal metabolic function and light activity of the average-weight person in his or her age and sex group is considered food energy deficient. However, because we do not know each person's actual requirement (for basal metabolic function and light activity), and because in each age and sex group there is actually a range of requirements that may apply to individuals, there will be some classification error. Some people whose actual requirement is below the average might have an energy consumption level below the average requirement but still be meeting their own individual requirement. Similarly, some people whose actual requirement is above the average might have an energy consumption level above the requirement but below their own individual requirement. For estimating population prevalences, if these two groups are roughly the same size, the errors cancel each other out. Whether they are the same size is also a subject for future research.

\section{Analysis Is at the Household Level, with Intra- household Distribution Not Accounted For}

A final issue pertains to the measurement of the percentage of people, as opposed to households, who are food energy deficient. What is actually being measured is whether the energy availability 
of a household falls below its energy requirement, not whether that available to each member falls below her or his own individual requirement. If food is not distributed according to need within households, some people may be living in households classified as having a food energy surplus who are, in fact, not meeting their requirements. Similarly, some people may be living in households classified as food energy deficient who are nevertheless meeting their requirements. If these two "error" groups are not of the same size, there will be inaccuracies in the estimation of the percentage of people who are food energy deficient in a population group. 


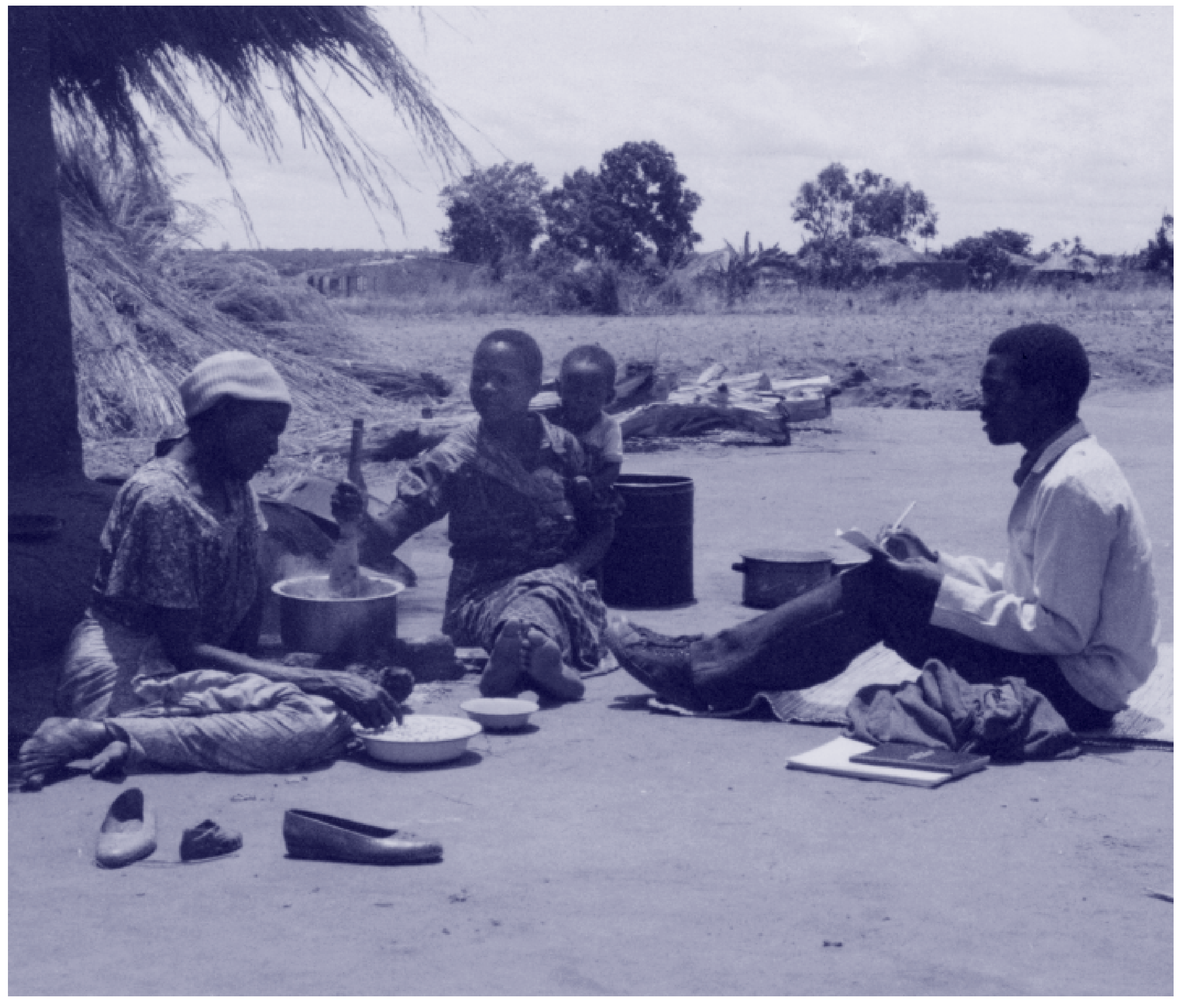




\section{Collecting the Food Data from Households}

$\mathrm{T}$ he primary objective in designing the food module of a HES questionnaire is to collect data needed to compute the metric quantity and monetary value of all foods acquired by households over a specific time period. This chapter discusses the various issues in questionnaire design, including the cost and accuracy trade-offs of various alternatives. It then reviews some of the more practical aspects of questionnaire design and survey administration. Note that two important questionnaire design choices that need to be made are (1) whether the interview or diary approach will be used and (2) whether to collect data on foods acquired or foods consumed. In the sections leading up to discussions of these choices, we assume that the interview technique is being employed to gather data on foods acquired.

\section{STRATEGIES FOR COLLECTING DATA ON QUANTITIES OF FOODS ACQUIRED}

\section{Foods Acquired for In-Home Consumption}

For foods destined for consumption, and thus preparation, inside the home, the goal is to measure the quantities of the foods in the form in which they were acquired before any additional preparation or processing (for example, soaking or cooking) is done. ${ }^{2}$ In many cases these are raw foods, but some foods may be processed as acquired, for example, flours and margarine.

A primary consideration in designing the module for collecting the data is how quantities of food in standard units of weight (usually kilograms or grams) will eventually be obtained. It is important to carefully think through in advance the best way to get an accurate estimate of quantities acquired from survey respondents in any given location. Usually the food acquired has already been partially or wholly consumed at the time of an interview, so it is not possible to actually view and measure it directly by weighing it with a scale. Respondents must estimate the amount acquired, and to do so should be familiar with and comfortable with the units of measure in which they report.

In this section we discuss seven methods of measuring food quantities that will eventually be converted to metric units. They are listed in Box 1.

In general, previous HESs have applied a common approach to all foods for which data were collected. They have mainly relied on the first four methods, using either Method 1 or 2 or some combination of Methods 3 and 4 . We recommend instead that a food-specific approach, informed by a strategy for converting the quantity of each individual food to metric units, be adopted. This is because different methods are best suited to different foods, as discussed in this section.

2 This section draws on Gibson and Ferguson (1999), Swindale and Ohri-Vachaspati (2004), and Smith, Alderman, and Aduayom (2006). 


\section{Box 1. Methods of Collecting Data on Food Quantities}

1. Metric units. Respondents report directly in metric units.

2. Monetary value. Respondents report monetary values of foods (expenditures), which are later divided by a metric price.

3. Local units of measure. Respondents report quantities in nonmetric, or "local," units of measure (for example, cans, heaps), which are later multiplied by a metric weight conversion factor.

4. Counts. Respondents report quantities in terms of numbers of individual food items, which are later multiplied by a metric weight conversion factor.

5. Volumetric equivalents. Respondents demonstrate how much space the quantity of food takes up, which is later multiplied by a metric weight conversion factor.

6. Linear dimensions. Respondents demonstrate the length, width, or circumference of the quantity of food, which is later multiplied by a metric weight conversion factor.

7. Food models. Respondents point to a model of the quantity of the food, which is later multiplied by a metric weight conversion factor.

The primary considerations that should be taken into account when choosing a method of collecting data on the quantities of a specific food are

1. the importance of the food in the population's diet;

2. the accuracy of measurement of quantities of the food using the method;
3. whether respondents are comfortable reporting in the unit of measure on which the method relies;

4. the time required by survey respondents, interviewers, and data processors; and

5. whether equipment beyond the survey questionnaire and a recording instrument is needed.

Accurate measurement is especially important for those foods deemed most important in the diet of the surveyed population. However, greater accuracy usually comes at a cost in terms of greater time required to conduct household interviews, collect additional data from other sources, and process the data. The degree to which respondents feel comfortable reporting in a given unit of measure can be determined during pretesting of the questionnaire. It is best to allow them to choose the measure in which they will report for each food. Any additional equipment needed to conduct the interview (beyond the questionnaire and a pen or pencil) must be provided for each interviewer and transported from household to household, increasing both the cost of the survey and the burden on interviewers.

Tables 3 and 4 outline the cost and accuracy considerations to take into account for each of the seven methods of collecting data on food quantities listed in Box 1. Table 5 lists some commonly consumed foods and the methods that can be used to measure quantities of them acquired.

1. Metric units. Respondents are asked to report quantities directly in metric units of measure (for example, kilograms or liters) along with expenditures for each food. ${ }^{3}$ This is one of the lowest-cost methods of collecting the food data.

3 This method applies to all standard units of measure, even those that are nonmetric (for example, pounds). 
Table 3. Choosing a method for collecting data on foods acquired for in-home consumption: Cost considerations

\begin{tabular}{|c|c|c|c|c|}
\hline Method & $\begin{array}{l}\text { Data to report and record during } \\
\text { household interview }\end{array}$ & $\begin{array}{l}\text { Equipment needed for } \\
\text { household interview }\end{array}$ & $\begin{array}{l}\text { Additional data needed to convert } \\
\text { to metric weights }\end{array}$ & $\begin{array}{l}\text { Data cleaning and } \\
\text { processing steps }\end{array}$ \\
\hline 1. Metric units & $\begin{array}{l}\text { Vocal reporting of quantity and } \\
\text { expenditure }\end{array}$ & None & $\begin{array}{l}\text { Volume-weight conversion } \\
\text { factors (only for foods reported in } \\
\text { volumetric units), one for each food }\end{array}$ & Clean unit values (UVs). \\
\hline $\begin{array}{l}\text { 2. Monetary } \\
\text { value }\end{array}$ & Vocal reporting of expenditure & None & $\begin{array}{l}\text { Metric food prices (collected at } \\
\text { community level or higher) }\end{array}$ & $\begin{array}{l}\text { Clean price data. } \\
\text { Calculate metric quantities. }\end{array}$ \\
\hline \multicolumn{5}{|l|}{$\begin{array}{l}\text { 3. Local units } \\
\text { of measure }\end{array}$} \\
\hline Variable size & $\begin{array}{l}\text { Vocal reporting of quantity, unit of } \\
\text { measure, and expenditure }\end{array}$ & None & $\begin{array}{l}\text { Metric weights of foods in local } \\
\text { units (community level or higher) }\end{array}$ & $\begin{array}{l}\text { Clean metric weights. } \\
\text { Calculate metric quantities. } \\
\text { Clean UVs. }\end{array}$ \\
\hline Fixed size & $\begin{array}{l}\text { Vocal reporting of quantity, unit of } \\
\text { measure, and expenditure }\end{array}$ & None & $\begin{array}{l}\text { Metric weights of foods, one for } \\
\text { each food }\end{array}$ & $\begin{array}{l}\text { Calculate metric quantities. } \\
\text { Clean UVs. }\end{array}$ \\
\hline 4. Count & $\begin{array}{l}\text { Vocal reporting of quantity, unit of } \\
\text { measure, and expenditure }\end{array}$ & None & $\begin{array}{l}\text { Metric weights of foods (community } \\
\text { level or higher) }\end{array}$ & $\begin{array}{l}\text { Clean metric weights. } \\
\text { Calculate metric quantities. } \\
\text { Clean UVs. }\end{array}$ \\
\hline $\begin{array}{l}\text { 5. Volumetric } \\
\text { equivalents }\end{array}$ & $\begin{array}{l}\text { Demonstration reporting of quantity; } \\
\text { vocal reporting of expenditure }\end{array}$ & $\begin{array}{l}\text { Calibrated container(s), } \\
\text { rice, water }\end{array}$ & $\begin{array}{l}\text { Volume-weight conversion factors, } \\
\text { one for each food }\end{array}$ & $\begin{array}{l}\text { Calculate metric quantities. } \\
\text { Clean UVs. }\end{array}$ \\
\hline $\begin{array}{l}\text { 6. Linear } \\
\text { dimensions }\end{array}$ & $\begin{array}{l}\text { Demonstration reporting of quantity; } \\
\text { vocal reporting of expenditure }\end{array}$ & Ruler & $\begin{array}{l}\text { Size-weight conversion factors, one } \\
\text { for each food }\end{array}$ & $\begin{array}{l}\text { Calculate metric quantities. } \\
\text { Clean UVs. }\end{array}$ \\
\hline 7. Food models & $\begin{array}{l}\text { Demonstration reporting of quantity; } \\
\text { vocal reporting of expenditure }\end{array}$ & $\begin{array}{l}\text { Two- or three- } \\
\text { dimensional food models }\end{array}$ & $\begin{array}{l}\text { Size-weight conversion factors, one } \\
\text { for each food }\end{array}$ & $\begin{array}{l}\text { Calculate metric quantities. } \\
\text { Clean UVs. }\end{array}$ \\
\hline
\end{tabular}

Note: Clean unit values (UVs) are calculated as expenditure divided by quantity. Further detail on UV cleaning is found later in this guide. 
Table 4. Choosing a method for collecting data on foods acquired for in-home consumption: Accuracy considerations

\begin{tabular}{|c|c|c|c|}
\hline Method & Pros & Cons & When method can be used \\
\hline 1. Metric units & $\begin{array}{l}\text { Can use unit value (UV) } \\
\text { cleaning to check for major } \\
\text { errors }\end{array}$ & $\begin{array}{l}\text { Respondents may not be able to report } \\
\text { in metric units for most foods }\end{array}$ & $\begin{array}{l}\text { When respondents obtain a food in metric units or are } \\
\text { very familiar with its metric weight }\end{array}$ \\
\hline 2. Monetary value & $\begin{array}{l}\text { May yield reasonably } \\
\text { reliable population group } \\
\text { estimates (at low cost) }\end{array}$ & $\begin{array}{l}\text { Major errors at the household level } \\
\text { possible: prices not matched with } \\
\text { households; no UV cleaning }\end{array}$ & $\begin{array}{l}\text { When the food has a fixed or very stable price across } \\
\text { households }\end{array}$ \\
\hline \multicolumn{4}{|l|}{$\begin{array}{l}\text { 3. Local units of } \\
\text { measure }\end{array}$} \\
\hline Variable-size & $\begin{array}{l}\text { Can use UV cleaning to } \\
\text { check for major errors }\end{array}$ & $\begin{array}{l}\text { Errors at household level possible: } \\
\text { weights not matched with households, } \\
\text { but UV cleaning helps detect }\end{array}$ & \\
\hline Fixed-size & $\begin{array}{l}\text { Can use UV cleaning to } \\
\text { check for major errors }\end{array}$ & $\begin{array}{l}\text { Greater precision gives greater } \\
\text { accuracy than for variable-size units of } \\
\text { measure }\end{array}$ & \\
\hline 4. Count & $\begin{array}{l}\text { Can use UV cleaning to } \\
\text { check for major errors }\end{array}$ & $\begin{array}{l}\text { As for Method 3, variable-size units of } \\
\text { measure }\end{array}$ & $\begin{array}{l}\text { When the food can be counted (individual units are not } \\
\text { too small) and varies little in size }\end{array}$ \\
\hline $\begin{array}{l}\text { 5. Volumetric } \\
\text { equivalents }\end{array}$ & $\begin{array}{l}\text { Can use UV cleaning to } \\
\text { check for major errors }\end{array}$ & $\begin{array}{l}\text { Greater precision gives greater } \\
\text { accuracy than Methods } 3 \text { and } 4\end{array}$ & $\begin{array}{l}\text { When the food can be poured into a container that is not } \\
\text { too large (for example, }<1 \text { liter) (liquids or solids with } \\
\text { small pieces) }\end{array}$ \\
\hline $\begin{array}{l}\text { 6. Linear } \\
\text { dimensions }\end{array}$ & $\begin{array}{l}\text { Can use UV cleaning to } \\
\text { check for major errors }\end{array}$ & As for Method 5 & When the food has a rectangular or circular shape \\
\hline 7. Food models & $\begin{array}{l}\text { Can use UV cleaning to } \\
\text { check for major errors }\end{array}$ & As for Method 5 & $\begin{array}{l}\text { For solid foods that vary greatly in size and are difficult to } \\
\text { measure with any precision using the other methods (for } \\
\text { example, fish, roots, and tubers) }\end{array}$ \\
\hline
\end{tabular}

Note: Clean unit values (UVS) are calculated as expenditure divided by quantity. Further detail on UV cleaning is found later in this guide. 
Table 5. Summary of methods of data collection suitable to different types of foods

\begin{tabular}{|c|c|c|c|c|c|}
\hline Type of food & $\begin{array}{l}\text { Metric units, monetary value, } \\
\text { and local units of measure }\end{array}$ & Count & $\begin{array}{l}\text { Volumetric } \\
\text { equivalents }\end{array}$ & Linear dimensions & Food models \\
\hline $\begin{array}{l}\text { Cereal grains (threshed), } \\
\text { flours, meals, sugar, salt, } \\
\text { powdered milk }\end{array}$ & $x$ & & $x$ & & \\
\hline Roots and tubers & $\mathrm{X}$ & $\begin{array}{c}\mathrm{X} \\
\text { (not recommended) }\end{array}$ & & $x$ & $x$ \\
\hline $\begin{array}{l}\text { Commercial baked goods (for } \\
\text { example, bread), candy, gum }\end{array}$ & & $x$ & & $x$ & $x$ \\
\hline Pastas & $x$ & & $x$ & & $\mathrm{X}$ \\
\hline Pulses, most nuts, seeds & $x$ & & $x$ & & \\
\hline Peanut butter, tomato paste & $x$ & & $\mathrm{X}$ & & $\mathrm{X}$ \\
\hline Cheese & $x$ & & & $x$ & $x$ \\
\hline Yogurt & $x$ & & $x$ & & \\
\hline Eggs & $x$ & $x$ & & & $x$ \\
\hline Meat and meat products & $x$ & $\begin{array}{c}\mathrm{X} \\
\text { (not recommended) }\end{array}$ & & $x$ & $\mathrm{x}$ \\
\hline Fish & $x$ & $\begin{array}{c}\mathrm{X} \\
\text { (not recommended) }\end{array}$ & & $x$ & $x$ \\
\hline Shellfish & $x$ & $x$ & & $x$ & $x$ \\
\hline Fruits, vegetables, coconuts & $x$ & $x$ & & $x$ & $x$ \\
\hline $\begin{array}{l}\text { Liquids, oils, fats, honey, } \\
\text { syrups }\end{array}$ & $x$ & & $x$ & & \\
\hline
\end{tabular}

Note: $X$ indicates that the method can be used to measure quantities of the food 
No additional data are needed beyond those collected from households except for volume-to-weight conversion factors for those few foods that would be reported in volumetric units (for example, beverages in liters). No equipment is needed. Data processing involves cleaning of household-level unit values (expenditures divided by quantity; see the fourth section of Chapter 5) to detect errors in the quantity and expenditures data. However, this is a viable method only for foods that are commonly purchased or otherwise obtained in a metric weight (for example, weighed on a scale at the time of purchase) or volume that is well known to respondents or can be directly recorded from the container in which the food was obtained. For most populations this is not the case, and thus this method is simply infeasible from an accuracy standpoint.

It is important to keep in mind that in some countries respondents may imply that a product has been weighed and report a standard unit weight when, in fact, the product was not weighed. For example, in the Dominican Republic vendors measure beans in a specific-sized can, and a full can is called "one pound." However, the beans in the can actually weigh about three-quarters of a pound (Swindale and OhriVachaspati 2004). In many countries people purchase grains in sacks that originally contained a different product yet report the weight written on the sack. For example, sorghum may be purchased in a 25-kilogram rice bag, which can lead to erroneous reporting of 25 kilograms of sorghum.
2. Monetary value. Respondents are asked to report only their expenditures on each food. This is also one of the lowest-cost methods. During the household interview itself, the burden on respondents and interviewers is the slightest of all the methods because only one piece of information is being recorded for each food. Unlike in Method 1, additional data collection at the community or higher (district, region) levels is required beyond the household interview, that is, data on metric prices used for converting expenditures to metric quantities. Although the price data must be cleaned and the conversion must take place at the data processing level, no time-consuming cleaning of household-level unit values is undertaken. Note that in some cases metric prices for districts or regions within a country are available from other surveys, such as consumer price index surveys, which would lower costs. The prices must be for the same time period and the same foods as the HES, which is often not the case.

There has been no research to date comparing estimates of food security indicators based on this method with those based on methods in which quantity data are collected directly. Household-level estimates of food expenditures can suffer from considerable inaccuracy for a number of reasons. In rural areas some foods may not be bought or sold on markets, so respondents may find it difficult to place a monetary value on the amount of the food acquired. ${ }^{4}$ Also, food prices can vary greatly across households due to differences in quality, the amount of the food purchased at a given time (that is,

${ }^{4}$ In these cases it may be possible to estimate a "barter equivalent value" by asking respondents to report for how much of an alternate food that can be easily valued they would trade the amount of the food in question. 
whether it is a bulk purchase), or the purchaser's negotiating skills and personal relationships with vendors. Furthermore, because there are no unit values to calculate and clean, errors are often undetectable.

If cost is a major concern, this method may give reasonably reliable estimates of food security indicators at lower cost than most other methods. However, we recommend that it be used only for foods with fixed prices or whose prices do not vary much across households. This is sometimes true for commercially packaged foods (for example, sugar, milk, soft drinks, foods in cans). In these cases, estimates of householdlevel food quantities are reasonably accurate, and collection of expenditure data may be the best method to use for practical reasons.

3. Local units of measure. Respondents report food quantities in terms of the number of units of measure in which foods are commonly acquired or used (most often nonmetric units), along with expenditures on each food. The respondent indicates either how full the unit was at the time of acquisition or, if full, the number of units. This method is more costly than the first two. In addition to three pieces of information on each food collected from households — quantity, unit of measure (unless prespecified), and expenditure—data must be collected at the community or higher level on the metric weight of each food in each unit of measure in which it is reported. Although no equipment is necessary for the interview, more time is required for data cleaning and processing. The metric weight data must first be cleaned. Next, where a unit of measure is not prespecified, the recorded units of measure must be checked for miscoding. The metric quantities must then be calculated and cleaned by examining household-level unit values for outliers.

The accuracy achieved with this method depends on how much the size of a unit of measure varies across households. The greatest degree of accuracy is achieved when the unit of measure has a fixed size. Examples are sacks and cans that originally contained a specific quantity of a specific food. For example, in the Papua New Guinea survey analyzed as part of the AFINS project, a 25-kilogram rice bag was used as a unit of measure for reporting on various other food items, including bananas, peanuts, sweet potatoes, and yams. The Uganda survey used margarine tins of prespecified sizes $(1 / 2,1$, and 2 kilograms). Beverage quantities can be reported in terms of soda or beer bottles of commonly recognized sizes. The metric weights of various foods in these units of measure can be accurately estimated. Although it is not necessary, it is best if the respondent can see the actual unit in mind to reduce the chance of confusion (in which case equipment is required for this method). For example, sacks of the two most commonly used sizes can be carried by enumerators.

Some commonly used units of measure can vary in size across households but are the only units in which respondents can report. Examples are bundles, heaps, bunches, glasses, cups, bowls, and plates. Because of size variations by household, only rough estimates of the metric weights of food items in these units of measure can be obtained. Some units of measure that are very large and have widely varying sizes should not be used at all unless a commonly understood specific size can be attached to them (for example, sacks, baskets, and calabashes). 
Appendix 2 lists the local units of measure used for measuring quantities of different foods in the AFINS project surveys.

4. Count. Respondents report the number of individual pieces of foods acquired. The units of measure are referred to as "unities" (they can also be called "pieces" or "singles"). This method generally has the same cost and accuracy trade-offs as Method 3. The sole advantage of this method is that it may be easier for respondents to think about counting a tangible food (for example, a mango) that has recently been acquired than reporting in terms of the more abstract units of a container holding a given food item. It can be used only for specific kinds of foods, that is, those that can be acquired in their entirety and are big enough in one contiguous piece to be counted. It is best used for items for which there is little variation in size (and thus weight). In some cases greater accuracy can be achieved if the respondent specifies whether the item is "small," "medium," or "large," even though the definitions of these qualifiers may be somewhat ambiguous.

The foods that can be reasonably accurately measured in counts are eggs, shellfish, fruits, vegetables, and some commercial baked goods such as bread or slices of bread. Foods such as roots, tubers, meat, meat products, and fish can be measured in counts, although it is not recommended due to wide variations in terms of size and thus weight.

5. Volumetric equivalent units. Respondents demonstrate how much space the food takes up in a calibrated volumetric container, either by pointing to the level to which the container would be filled if the original food were placed in it or by actually filling it up with rice or water to that level. The interviewer then reads off the amount from the container. If more than one container was acquired, the respondent can tell the interviewer how many were acquired.

Interview time is increased with this method, because respondents must physically demonstrate the amount of space the acquired food takes up, which requires more time than vocal reporting. However, only one volume-to-weight conversion factor is needed for each food. The conversion factor for each food item can then be used for multiple communities or geographical areas. On the other hand, the method requires that equipment be brought to interviews. Specifically, calibrated measuring containers, such as cups, spoons, and see-through measuring cylinders or beakers of various sizes are needed, depending on which foods are designated to be measured using this method. The data cleaning and processing burden is less than for Methods 3 and 4, because the conversion factors do not need to be cleaned.

This method is somewhat more accurate than Method 3, even when the latter involves units of measure of a fixed size, because respondents can report with greater precision. This is especially so when the amount required is less than one unit of measure, in which case the respondent needs to estimate a fraction. The method is likely to be far more accurate when the units of measure are of a variable size, for example, heaps and bowls.

This method is useful for foods that can easily fill up all of the space in a container. Examples are liquids, grains (for example, rice, maize, millet, and sorghum), flours, beans, seeds, sugar, and powdered milk. 
6. Linear dimensions. Respondents demonstrate the length, width, or circumference of the food acquired by identifying a point along on a calibrated measuring stick (a ruler). Because it is a demonstration rather than a vocal method, the interview time is likely to be longer than for the first four methods. As in the volumetric equivalent method, only one piece of additional data must be collected for each food to convert the data collected from households to a metric weight equivalent, and conversion factor data cleaning is not necessary.

This method can be used when an item has a square or rectangular shape or varies mostly in only one dimension. It is suitable for measuring the size of some roots and tubers, commercially baked goods (for example, bread and tortillas), cheese, some meats, fish and shellfish, fruits and vegetables (for example, carrots), and sugarcane. Because recording errors can be a problem with this method, good enumerator training is very important.

7. Food models. Respondents point to the two- or threedimensional model of a food that best corresponds to the amount of the food that was actually acquired or, if only one model size is available, report on the size acquired in relation to the model size and the number acquired. ${ }^{5}$ Due to the costs of assembling metric weights and preparing models of foods, this method is usually too costly to implement in an HES. However, if a food is a very important part of the diet and the quantities acquired cannot be measured accurately using the other methods, it may be worth the investment. For the greatest precision, it is best to have a range of common sizes and shapes for respondents to choose from. However, if it is possible to have only one, respondents will need to estimate the size in relation to the model (bigger, smaller, half the size, twice the size, and so forth). Where thickness is a factor (for example, with tortillas), cardboard models for thickness can be used.

In some cases plasticized color pictures (either photographs or drawings) may be best, because these can easily be carried by enumerators in the field.

Although this method can be used for a variety of foods (see Table 5), in the interest of cost savings it is recommended only for foods that vary greatly in size, and thus for which it may give the most accurate quantity estimates, such as fish and roots and tubers. These items are notoriously difficult to accurately measure using other methods.

\section{Foods Consumed Outside the Home}

Foods consumed outside the home are often prepared dishes containing multiple foods. Assessing their energy content requires estimating the quantities of each raw ingredient, which is a complex process. For this reason, most HES surveys have not included collection of data on the quantity or even the identity of foods and drinks prepared and consumed outside the home, for example, restaurant meals.

The common practice is to ask respondents to estimate only the total amount of money spent on food consumed outside the home. To

5 The use of clay models is a more advanced food model technique. Respondents shape clay into the size of the food acquired, and then metric conversion factors are estimated using the method of water displacement. See Swindale and Ohri-Vachaspati (2004). 
estimate the energy content of the food, the expenditure is divided by the price per calorie of food acquired for consumption in the home. ${ }^{6}$ Estimates of diet diversity are based solely on the foods acquired for in-home consumption.

Although this method is a very low-cost option, estimates of food security indicators based on it can be highly inaccurate, especially for populations for which food consumed outside the home is an important part of the diet. First, people may eat different kinds of food, with different energy values, in the meals they consume outside their homes compared to inside. Second, expenditures for the same quantities of foods consumed outside of the home are likely to be higher than for those consumed inside because of the added charges for preparation labor. Third, meals eaten outside the home may contain food from a wider variety of food groups than those eaten inside, in which case diet diversity measures are biased downward.

Food prepared outside of the home is an important part of the diet in many countries and will become increasingly so in others over the next few decades, especially in urban areas. It is thus important to collect additional data that will allow more precise estimates of the quantities of foods acquired this way, especially for populations for which they are already an important part of the diet. In the case of raw foods or single-ingredient processed foods (such as bread, tortillas, or pasta), the same techniques as those described earlier for foods acquired for in-home consumption can be used.

In the case of a prepared dish containing multiple ingredients, a technique with reasonable accuracy that poses little burden on households is recommended (see Box 2). Only two pieces of
Box 2. Recommended Method for Collecting Data to Calculate Quantities of Foods in Prepared Dishes

1. Ask respondents which dishes were consumed and their monetary value.

2. Collect information from vendors on the amounts of ingredients used to prepare each dish and the metric price of each dish.

3. Calculate the proportionate weight of the ingredients in each dish.

4. Use the price per gram of the dishes to estimate the weight of ingredients consumed based on the monetary values reported by the households.

information are needed from households: the identity of the dishes consumed and their expenditure on the dish. The added burden of the method lies in the fact that estimation of metric quantities of ingredients requires gathering additional information from vendors on the ingredients of dishes and their prices (outlined in the third section of Chapter 4).

To reduce costs (but, as usual, with a reduction of accuracy as well), the technique can be applied to a random subsample of households. The data can then be used to compute factors for adjusting price-per-calorie estimates computed from food acquired for in-home use to estimate the energy content of foods acquired for outside consumption. It is important to compute separate factors for urban and rural households and income groups, for which price-percalorie estimates are likely to vary substantially.

${ }^{6}$ In some cases the price-per-calorie of food consumed at home is multiplied by a mark-up to account for the higher value due to processing and convenience. For example, Subramanian and Deaton (1996) using data from rural India apply a 50 percent mark-up to account for processing. 
At a minimum, respondents should be asked about the identity of the dishes consumed in addition to their total expenditures on all foods consumed outside the home. Collection of data from vendors on the ingredients of these dishes, if they are not already available from secondary sources, would allow foods consumed away from home to be accounted for in diet diversity indexes.

\section{OTHER CHOICES TO BE MADE IN QUESTIONNAIRE DESIGN}

\section{Whether to Survey Foods Acquired or Foods Consumed}

Some previous surveys have attempted to collect data on foods consumed by households rather than acquired. In the case of foods consumed from households' own production, the amounts of foods acquired and consumed will be the same, but for foods purchased and received in-kind, they can differ. ${ }^{7}$ This is because, as noted in Chapter 2, households do not always eat everything they acquire over a specific time period and do not always acquire the food eaten over the period during the period itself (it is often acquired earlier and stored). Estimates of food security indicators are, in theory, roughly the same for population groups, whether data on food consumption or food acquisition are collected. However, when data are collected on food consumption in the context of HESs, the reporting error is likely to be greater than for food acquisitions for a number of reasons.
First, when reporting quantities of foods consumed, respondents must undertake a number of complex calculations and report estimates rather than actual amounts associated with single events. This is because the recall period is usually at least seven days and the focus in HESs is on raw foods as acquired rather than on prepared dishes. In answer to the question "How much did you consume of $\mathrm{X}$ over the last week?" respondents must think of all the events of eating the food (which is often included in a prepared, multipleingredient dish) involving the food over seven days. When the quantity consumed is less than the quantity acquired, the respondent must think back to the ingredients used in dishes that may have been prepared and consumed multiple times. Note that household food consumption surveys, which have a recall period of one day, are specifically designed for collecting these kinds of data using more in-depth interviewing techniques and more precise measurement techniques (Swindale and Ohri-Vachaspati 2004).

Second, while the event of consuming a food may have taken place during the recall period, the event of acquiring it may have taken place much earlier, which complicates reporting of expenditures on food purchases. Respondents may have good recall of the recent event of consuming the food, yet to properly report expenditures on food purchases they must rely on their memory of the original purchase event. Thus, the recall period can effectively be extended, inviting increased recall bias. Expenditures may not be properly matched with quantities, leading to difficulties in later analysis, such as estimating prices based on unit values and estimating the relationship between total expenditures and the

7 It is important to note that in this manual we define the food acquired from one's own production as only the food that is actually consumed by household members and not any that is harvested but not consumed. 
consumption of various foods. This is especially so when the quantity consumed is less than the quantity acquired, which requires that respondents calculate a fraction in their heads.

When data are collected on food acquisitions, in contrast, respondents are asked to report on the quantity obtained over the recall period. The number of events in which a food is obtained is likely to be smaller than the number of events in which it is consumed. This is because a household may acquire a food and then use it over multiple events, but it usually will not acquire the same food several times and only then eat it. Thus, there are likely to be fewer calculations in which the respondent must add up amounts over multiple events. In the case of expenditures, a purchase event will have occurred during the recall period and the respondent will more easily remember the total amount spent, giving an exact value from memory rather than undertaking estimations over multiple uses of the food.

Note that many HES questionnaires include a "filter" question asking respondents whether individual foods have been consumed over the recall period and then return to ask them about the quantities purchased and so forth over the same period; that is, they are in effect subsequently asked about food acquisitions. This leads to errors in the reporting of both food acquisitions and consumption. Foods that were acquired during the recall period but not consumed are not accounted for at all. For foods that were consumed but not acquired, households can report zero quantities and expenditures or try to come up with estimates of the portion of the food acquired that was actually consumed, which, as noted earlier, invites further error and a mismatch between quantities and expenditures.

\section{Which Foods to Include}

HES interview questionnaires include a preselected list of food items for which data are collected. ${ }^{8}$ Interviewers go through the list of foods one by one to collect data, which can be very time-consuming. In the interest of accuracy, one seemingly obvious goal in constructing this list would be to include all individual food items that could possibly be acquired by households, that is, to make an exhaustive list of foods with the greatest detail possible. However, the longer the list, the more time it takes to administer the survey, the more likely respondents and interviewers are to become fatigued and report or record incorrectly, and the longer it takes to process the data after they are collected. Thus, this approach, when taken to an extreme, may not only be very costly but also compromise accuracy (Deaton and Grosh 2000). On the other hand, as experiments conducted in El Salvador, Jamaica, Ecuador, and Indonesia show, although shorter questionnaires reduce survey costs, they likely reduce the amount of food expenditures reported and thus also compromise accuracy (Deaton and Grosh 2000, Pradhan 2001). The smallest number of food items included in the AFINS project surveys is 33 (for Burundi), and the highest is 346 (for Malaysia). A suggested minimum number of food items for obtaining accurate food security estimates is 40 .

One technique that is used in almost all HESs to reduce the size of the food list is to list only the most commonly acquired foods in broader food categories and then at the bottom of the list include an "other" category. For example, the most common fruits are listed followed by a category headed "Other fruits." Depending on the cost constraints and degree of accuracy desired, interviewers can be

8 Allowing households to list foods on their own is not recommended, because they are likely to leave out many food items acquired. 


\section{Box 3. Basic Principles for Constructing a Food List}

1. List similar foods with differing caloric contents or edible portions as distinct items so that proper conversion to energy content can take place. Some examples are

\section{cereals in their grain and flour forms:}

paddy and husked rice;

plantains and sweet bananas;

maize cobs and maize grain;

fresh and dry beans:

shelled and in-shell groundnuts and seafoods;

fresh, dried, and smoked fish;

unboned and deboned meats, ${ }^{\text {a }}$

fresh and dry peppers;

sweetened and unsweetened condensed milk;

different kinds of cheese;

beer, wine, and distilled beverages in the category "alcohol";

and

baby foods and formula.

2. List different versions of the same food with greatly differing prices separately so that proper conversion to metric quantities (if the "monetary value" method is used), unit value cleaning, and analysis of price effects can be undertaken. Examples are

foods that are both produced locally and imported, such as rice, maize, sugar, salt, and alcoholic beverages;

foods purchased at both subsidized and unsubsidized prices; foods that come in different qualities (for example, maize meal in Zambia):

foods sold both canned and uncanned; and

foods in their raw and processed forms.

3. List food and nonfood items separately. In particular:

Do not list alcohol and tobacco together in one category.

Do not list expenditures in "restaurants and hotels" in one category (the latter includes lodging).

4. Do not include catch-all residual categories that do not allow food identification, for example, "Miscellaneous food items,"

"All other foods," or "Other canned foods."

a If it is known in advance that households obtain meat in one state or the other, it is not necessary to make this distinction in the questionnaire.

instructed to ask respondents to specify the identity of these other food items and collect the same data as for the prelisted foods.

Another way to save time is for interviewers to first ask whether any foods in a broader category (for example, fruits) have been acquired over the reference period before asking about the individual food items within each category. However, this may reduce accuracy, because households may be more likely to remember a food item acquired if it is specifically asked about. Whether this is feasible would need to be determined for each individual population during questionnaire pretesting.
Keeping all this in mind, there are four basic principles to follow when constructing a food list; they are listed in Box 3. The more important a food is in the diet, the more important it is to adhere to the first two principles. If a group of similar foods are not important in the diet and have similar energy contents and prices (for example, leafy greens in some areas), it may make sense to list them as one food item, with little reduction in accuracy of food security indicators.

The food list should be constructed based on the food consumption patterns of the population. It possible, food lists from recent surveys, including food consumption surveys, should be the 
starting point. If none is available, it may be necessary to conduct a survey of foods consumed (for example, in the previous day) for households or focus groups spread throughout the survey area to find out which foods are commonly consumed. It is important to include gathered wild fruits, vegetables and roots, wild animals hunted or fished, and the blood of animals in the food list if they are important in the diet. Keep in mind that food items consumed in rural and urban areas may greatly differ.

The convention is to list botanically similar food items together, listing first within each group the items that are more commonly consumed. This probably helps respondents to better remember what they have acquired (Deaton and Grosh 2000). There are a number of databases of food classifications and descriptions (see Ireland and Moller 2000). FA0 (2006a) (available online) gives a useful food list and classification of foods with definitions of each food.

\section{Whether to Use a Diary or an Interview}

An accurately kept diary, where acquisitions of food are recorded by respondents on a daily basis, will reduce errors in reporting food quantities and expenditures, especially recall errors. It is also more likely to capture all of the foods that a household acquires than is an interview based on a questionnaire with preselected food items. This is evident from the large numbers of different kinds of foods recorded in diaries compared to reports from interviews.

However, in practice, a diary cannot be kept unless there is a literate person in the household. If not, which is often the case in developing countries, interviewers must visit households frequently to fill in the diary. In such cases, the interview rather than the diary method is being used, which may lead to respondent and interviewer fatigue due to multiple time-consuming visits. Additionally, given that the information needed is somewhat complex, even literate people may have problems filling out a diary form without supervision.

This is evident from the problems trained interviewers often have in correctly recording information on food quantities, units of measure, and values of foods acquired. Because literate respondents may not fill out the diary on a daily basis, and it may not be feasible for an interviewer to visit illiterate households daily, the recall period may actually be greater than one day. Further, some households may not keep the diary for the entire reference period desired. Given these factors, the costs of collecting data using the diary method may outweigh the benefits.

\section{What Recall and Reference Periods to Use}

The recall period for food data collection is the amount of time for which respondents are asked to remember their food acquisitions. The reference period is the total amount of time for which they are asked to report their food acquisitions. The only circumstance under which the recall and reference periods differ is when households are interviewed more than one time. For example, households may be visited four times to ask about their food acquisitions in the last 7 days. In this case, the recall period is 7 days and the reference period is 28 days.

The longer the recall period, the more difficult it will be for respondents to remember the foods their household acquired over the period. Estimates of the quantities of foods acquired, energy availability, and diet diversity may be downward biased if the 
recall period is too long. 9 If the objective is only to obtain accurate population group estimates of mean household food or energy acquisition over a long period, say a year, the recall period should be as short as possible, one day. Only one visit will be necessary, and the reference period will thus be one day as well. Interviews can be conducted randomly across households throughout the year so that seasonal variations can be captured for the population as a whole.

Accurate estimates of usual food and energy acquisition specific to each household are needed for any analysis relating food security indicators to other variables (for example, education or income). If obtaining estimates of usual food and energy acquisition is the objective, longer reference periods should be used (optimally one year). This is because food acquisition can fluctuate greatly from day to day, and thus only one day would not adequately represent usual acquisition. To keep the recall period reasonable, this will require multiple visits to every household throughout the year, which of course makes a survey much more costly. However, the additional cost of multiple visits may not be necessary to achieve the accuracy desired. Because households tend to try to maintain constant consumption of food throughout the year, measuring consumption over a shorter period of time may yield a sufficiently accurate picture of usual consumption for the purposes of most surveys (Deaton and
Grosh 2000). Further, a survey with multiple visits spread out over a long period of time may experience a large dropout rate..$^{10}$

Most HESs have adopted a recall period of two weeks or less. Any longer period would probably lead to unacceptable reporting error. A one-week period may have an advantage over two weeks in that it is easier for households to remember what has happened since the same day last week (for example, Thursday). The day of the week can help set up a specific "memory post" of the beginning of the recall period in respondents' minds, bounding the period. ${ }^{11} \mathrm{~A}$ recall period of two weeks is likely to be more fuzzy, unless a previous visit took place two weeks before the interview, which can help bound the recall period in respondents' minds.

A recent analysis of data from India's national survey shows that it is especially important to use shorter recall periods, such as one week, for foods whose acquisition tends to fluctuate greatly from day to day, for example (in India), vegetables, fruits, spices, and beverages. In this case respondents are likely to try to add up how much was acquired over the eating events or shopping trips that took place over the recall period (employing "episodic enumeration"), which is harder to do for a longer recall period. In the case of foods for which the quantities consumed are more regular and fixed over time, such as staple food cereals, this is not as important, because respondents

9 Another source of error is "telescoping," where a respondent includes events that occurred before the recall period, thus inflating estimates of household food acquisition. The shorter the recall period, the greater the likelihood of telescoping. In the specific case of food acquisitions, which are of high frequency and small size compared to nonfood acquisitions, recall bias is thought to be more of an issue than telescoping (Deaton and Grosh 2000).

${ }^{10}$ Using household expenditure survey data from China, Gibson, Huang, and Rozelle (2003) find that, at least in the case of measures of poverty and inequality, it may be possible to circumvent the loss in accuracy caused by shorter-than-annual reference periods by making use of the correlation between each households' expenditures from at least two separate visits in extrapolating to annual estimates.

${ }^{11}$ A visit a week before the interview (as has been implemented in many Living Standards Measurement Surveys), in which preliminary data are collected, helps to set this memory post. 
Table 6. Recall and reference periods used for food data collection in AFINS project surveys using the interview approach

\begin{tabular}{|c|c|c|c|}
\hline Country & Recall period (days) & Number of visits & Reference period (days) \\
\hline \multicolumn{4}{|c|}{ Sub-Saharan Africa } \\
\hline Burundi & 15 & 1 & 15 \\
\hline Guinea & $\begin{array}{l}\text { Urban: } 3 \\
\text { Rural: } 2\end{array}$ & $\begin{array}{c}\text { Urban: } 10 \\
\text { Rural: } 7\end{array}$ & $\begin{array}{l}\text { Urban: } 30 \\
\text { Rural: } 14\end{array}$ \\
\hline Kenya & 7 & 1 & 7 \\
\hline Mozambique & $\begin{array}{l}\text { First visit: } 1 \\
\text { Other visits: } 3\end{array}$ & 3 & 7 \\
\hline Rwanda & $\begin{array}{l}\text { Urban: } 3 \\
\text { Rural: } 2\end{array}$ & $\begin{array}{l}\text { Urban: } 10 \\
\text { Rural: } 7\end{array}$ & $\begin{array}{l}\text { Urban: } 30 \\
\text { Rural: } 14\end{array}$ \\
\hline Uganda & 7 & 1 & 7 \\
\hline Zambia & $\begin{array}{c}14 \\
\text { (for purchases of maize: } 30 \text { ) }\end{array}$ & 1 & $\begin{array}{c}14 \\
\text { (for purchases of maize: } 30 \text { ) }\end{array}$ \\
\hline
\end{tabular}

Asia

\begin{tabular}{lc|c|c} 
Bangladesh & $\begin{array}{c}2 \\
\text { (for spices: 7) }\end{array}$ & $\begin{array}{c}7 \\
\text { (for spices: 2) }\end{array}$ & 7 \\
\hline India & $7^{\text {a }}$ & 1 & 7 \\
\hline Indonesia & 7 & 1 & 14 (for 60 food items) and 30 (rest) \\
\hline Pakistan & 14 (for 60 food items) and 30 (rest) & 1 & 14 \\
\hline Papua New Guinea & 14 & 1 & 7
\end{tabular}

Note: The surveys were conducted throughout a full year except in Burundi (six months), Kenya (three months), Zambia (three months), and Indonesia (two months).

a Households were asked to report for both a 7-day and a 30-day recall period. The data from the 7-day recall period were used for the AFINS project analysis. 
estimate the quantities by first estimating for the typical day and then multiplying by the number of days in the recall period (Blair and Burton 1987, NSSO Expert Group 2003)

Table 6 gives the recall and reference periods used for the 13 AFINS project surveys for which the interview, as opposed to the diary, approach was used. Most of the surveys were based on a single visit, so the recall and reference periods are equal. The reference periods are thus mainly limited to one or two weeks. This is the case for the first nine countries. The rest of the surveys had multiple visits, from 3 to 10 , allowing shorter recall periods in most cases (from one to three days) and reference periods ranging from one week to one month. Note that in some surveys different recall or reference periods were used for urban and rural areas, different food sources (purchases versus homeproduced or received in kind), and specific subsets of foods.

\section{Methods to Use in Collecting Data on Food Expenditures}

There are three methods for collecting the data needed to calculate expenditures on each food acquired by households. The first is to directly ask respondents how much was spent on each food item acquired or, in the case of home-produced foods and foods received in kind (the "nonmonetary" sources), they are asked to estimate their total value. In the latter case, some questionnaires use the wording "How much would you have to spend to purchase that amount?"

The second method is to collect data on food quantities and then multiply them by metric prices collected as part of market price surveys or price opinion surveys (see the fourth section of Chapter 4). Although this method saves time, it gives less accurate expenditure estimates, because prices may vary across households. In recent surveys conducted in Malawi and Guatemala, respondents were asked to report their expenditures in addition to quantities when food was purchased, but only for quantities in the case of home-produced and in-kind foods. Presumably the unit values derived from the purchases data would then be used to calculate prices, which could then be used to compute expenditures for the nonmonetary food sources. If the surveyors rely on household-level unit values, it is important that the nonmonetary source foods also be purchased by some households in the same geographic area, which may not be the case for some foods, such as grains that are only home-produced (see the fifth section of Chapter 5 for more discussion of the use of unit values as estimates of prices).

The third method is to collect data from respondents on both the quantity and the price paid per local unit of measure for a food acquired and then to multiply the two. In past surveys using this method, there was a lot of confusion among interviewers and respondents alike as to what information was requested. Very often the recorded value was the total value of the acquisition rather than the value per unit. Thus, this method is not recommended unless it is determined during pretesting that the desired data are being collected. Note that the techniques used for price opinion surveys could be applied here (see the fourth section of Chapter 4).

\section{DRAFTING THE QUESTIONNAIRE}

There are a number of choices that need to be made in actually drawing up the food module of a questionnaire. ${ }^{12}$ Several drafts

${ }^{12}$ Much of the information in this section was drawn from Ferguson and Gibson (1999), Deaton and Grosh (2000), and Grosh, Glewwe, and Munoz (2000), in which more detailed discussions may be found 
may need to be drawn up, field tested, and reviewed by key users before finalization in order to ensure that the module is effective in collecting the necessary data. It may also need to be refined after interviewer training.

\section{Organization and Wording of the Questionnaire}

The first choice that must be made is how to organize the questionnaire. The primary objective in doing so is to collect all of the data needed (and only the data needed) in a way that elicits the most accurate responses and allows for recording of information in as little time as possible.

Data must be collected on

food purchases,

foods consumed from one's own production or gathered,

foods received in kind, and

foods consumed outside the home.

With respect to foods acquired for in-home consumption, the most time-saving way to organize the questionnaire is to include all three categories adjacent to one another on the same page, as in the model questionnaire found at the end of this chapter. This way, interviewers can ask about all three sources for each food item and will not need to go through the food list multiple times. However, some surveys have a separate submodule for home-produced foods, sometimes included in the "Agriculture" section of a survey. Only field testing can determine whether respondents find it easier to think about all of the sources of a single food together or in terms of the source. This likely depends on how important home-produced foods are in the population's diet.

Another issue to consider is whether to list the quantity or the expenditure question first. Most surveys place the quantity question first, followed by a column for recording the unit of measure and then the expenditure. This makes sense because a quantity is something that has physical dimensions and people may be able to more easily remember it because they can visualize it. This would then help them to remember the associated expenditure. However, for some populations this may make the respondent more likely to give the interviewer an answer about the household's expenditure per unit of quantity (because quantity has been asked for first) rather than for the total purchase. Field testing can determine which is best.

Information on foods acquired for out-of-home consumption cannot be collected alongside information on foods acquired from the other three sources, food by food. This is because most of these foods are in the form of prepared meals that will not correspond directly to the food listing, which contains mostly raw ingredients. It would seem logical to list the most common dishes eaten out of the home at the end of the food list, leaving space for additional dishes or individual foods not listed to be written in.

With respect to the wording of questions, rather than identifying only titles of what is to be entered onto the questionnaire (for example, "quantity" or "expenditure"), it is recommended that the actual questions that will be asked of respondents be printed out on the questionnaire and read out verbatim to respondents by the interviewer. Because different wordings may elicit different responses, this will ensure that questions are asked in a uniform way each time by all interviewers. The model questionnaire gives an example of this style. 
When wording questions, it is important to use terms that reflect the language of respondents as it is commonly spoken, not using a style that is too formal or academic. It is particularly important to make sure that the question about expenditures on food items is worded in such a way that respondents are not confused about whether to report a total value or a value per unit of purchase. It is also important that the question about the quantity consumed from one's own production be worded in such a way that respondents do not report their total stocks of a food or the total amount harvested over the recall period.

Instructions to interviewers written on the questionnaire should be short, concise reminders to jog enumerators' memories of what they learned in their training and what is in the interviewer manual. Such instructions should be clearly demarcated from text that they should read aloud to respondents. In the Living Standards Measurement Surveys (Grosh and Glewwe 2000), interviewer instructions are written in uppercase letters, while text to be read to respondents is written in lowercase letters. This is the style used in the model questionnaire.

\section{The Leading Statement}

Interviewers need to give some initial explanation to respondents as to what kind of data will be collected. Often this is laid out in the interviewer manual and perhaps practiced during training. However, it may be better to write it out at the top of the module to make sure that the information being given to respondents is uniform and correct. This statement should be short, to the point, and in language that is easily understood by respondents.
Respondents can usefully be informed about several things before beginning the data collection:

- They should be told that they will be asked about all of the foods "obtained" or "brought into the home" for consumption by household members over the recall period. This is by way of defining for them what is going to be asked. The questionnaire drafters should do their best to choose a word that is well understood by respondents. To avoid the reporting of foods entering the home for resale or trade, it is important to emphasize that the information being requested is about food for consumption by household members.

- They should also be told that questions will first be asked about the foods acquired for in-home consumption and the three sources of foods: purchase, home production or gathering, and receipt in kind. This is so that respondents will have it in mind that they will be asked to categorize, even for the first few foods.

- They should receive further explanation of the category "home produced," with instructions to include foods gathered, hunted, fished, or obtained from a household market enterprise (for example, a household-run shop).

- They should also receive further explanation of the category "in kind," with instruction to include gifts, foods received from employers, and donations of food.

- They should be informed that questions will then be asked about foods consumed outside the home.

- They should also be informed that data will be collected on foods that will be consumed by household members, not 
those that will be resold or used as an input into household enterprises (for example, as cattle feed).

The extent to which this information is needed depends on local conditions and can be determined during field testing of the questionnaire. Further explanation may need to be included in a leading statement if during pretesting respondents ask procedural questions of the interviewer or if the interviewer must correct the respondents.

\section{Filters, Skip Codes, and Block Outs}

"Filters," "skip codes," and "block outs" help save time by ensuring that respondents are not asked questions that are not applicable to them. This helps reduce respondent and interviewer fatigue, thereby improving accuracy in addition to saving time.

Filters are questions asked about an aggregate category to find out whether it is necessary to go into more detail. If not, a "skip code" can be added instructing enumerators as to which question to go to after the current question. Skip codes are helpful to interviewers because they keep them from having to make decisions themselves or to remember sometimes complicated rules printed in the interviewer manual. They also help to ensure that instructions will be followed uniformly by all interviewers.

Some possible filters for the food module are the following:

- Has any household member consumed food prepared outside the home over the recall period? If not, the subsection on food consumed outside the home can be skipped.

- Did the household acquire any food from its own production or gather it over the recall period? (This question might be used for survey populations or population groups where food consumed from their own production is not an important part of the diet.)

- Did the household acquire any "X," where $\mathrm{X}$ is a food category such as "fruits," over the reference period? If not, the interviewer may skip to the next food category.

- Did the household acquire any "X," where $X$ is a food such as "papayas," over the reference period? If not, the interviewer may skip to the next food.

The last two questions can be used for a complete enumeration of items consumed before asking the follow-up questions on quantities and/or expenditures so that respondents will not be tempted to say that they have not acquired something in order to shorten the interview by avoiding the follow-up question. This temptation is avoided because the enumeration is done before the respondent finds out that there will be follow-up questions on each item enumerated. Use of a food category skip may compromise accuracy due to the fact that respondents may not remember a specific food acquired within a category without a prompt from a detailed listing.

A block out saves time by blocking out in advance all recording areas on the questionnaire that do not apply to all households. In this case the interviewer knows not to ask the question. This may be done under the following circumstances:

- When a listed food item cannot be home-produced, in which case the home-produced blocks for quantities and units of measure and/or expenditure questions can be blocked out (for example, imported rice and bread in the model questionnaire).

- When the "monetary value" method for obtaining metric quantities is being used for a particular set or subset of foods, in 
which case the quantity and unit of measure questions can be blocked out.

- When expenditures are being estimated using reported quantities and prices collected in another part of the questionnaire or in a community price survey, in which case the expenditure question can be blocked out.

\section{Response Codes}

Response codes should be in local terminology and easily understandable by respondents. The potential questions in the food module with codable responses are those asking for unit codes and foods sources (for example, detailed in-kind sources) and those with various yes/no leading questions. As far as possible, all potential responses to each question with a codable response should be given a numeric code so that the interviewer can record only numbers, as opposed to words or phrases, on the questionnaire. Precoding allows the data to be entered into the computer straight from the completed questionnaire, thus eliminating the extra time needed and the errors that might accumulate from transcribing codes onto data entry sheets. The codes should be printed as close to the question as possible. In the case of unit codes, the list may be long. It may be possible to fit them onto the side (as in the model questionnaire) or the bottom border of each page of the module. But it may be necessary to place them on a separate page or on a note card to be carried by the interviewer and held alongside the questionnaire during an interview.

It is important that interviewers be aware of the appropriate uses of the unit of measure called "unities," which is used for the count method, and especially that they be able to distinguish it from the other units of measure. A mistake commonly made by interviewers is to code units of measure for foods that can be counted as being reported in unities when they are actually reported in other units of measure. For example, a household may report five heaps of potatoes, but the enumerator may code in five unities.

It is also important to always use the same food item name and code when referring to the same food item for different parts of the data collection process. This includes collection of data on food purchases and foods consumed from one's own production (in some surveys these are in separate modules), for which reported quantities and expenditures must eventually be summed. It also includes the collection of data on food prices and metric weights, which must eventually be matched with the data collected from households for conversion to metric quantities.

\section{CONDUCTING THE INTERVIEWS: SOME PRACTICAL POINTS}

A few practical points about conducting the interviews are as follows:

- The preferred respondent for the interviews should be the household member responsible for most food acquisition. This is often the person responsible for food preparation.

- It may be useful for interviewers to bring a card with them illustrating the food items in each category as a prompt. The illustrations can be either drawings or photos.

- Interviewers can be asked to encourage respondents to report in units of measure that are best for obtaining metric quantities. In a situation where a respondent reports in a unit of measure with a vague size, the interviewer can suggest an alternative 
with a standard size. For example, how many soda bottles of milk? How many margarine tins of flour?

- Interviewers can bring some essential props with them to the interview that will help respondents visualize quantities in a specific unit of measure with a standard size. For example, they can bring a cup and/or a tin with them to show respondents.

In the model questionnaire, interviewers are instructed to show respondents six units of measure.

- To avoid errors, interviewers should not undertake mathematical calculations in the field, whether in their heads or using calculators. The interviewers' key tasks should be to ask questions, listen to responses, and record them accurately.
In cases in which mathematical calculations are necessary, it is important to test staff for adequate mathematics skills, especially those responsible for quality control.

- As part of their training, interviewers should be instructed to be particularly careful to avoid showing signs of surprise, approval, or disapproval on learning about the respondent's eating pattern (Gibson and Ferguson 1999).

\section{MODEL HOUSEHOLD QUESTIONNAIRE}

The following is a model questionnaire on food acquisition. 


\section{MODEL SURVEY MODULE ON ACQUISITION OF FOOD OVER THE PAST WEEK}

I'm going to ask you some questions about the food brought into your home in the last week for your family to eat. I want to know about all the foods that you bought with money, ate from your farm or garden, or got from other people, such as friends, someone a family member worked for, or the government. Please tell me about a food even if you haven't eaten it yet.

\begin{tabular}{|c|c|c|c|c|c|c|c|c|c|c|c|c|}
\hline & 01 & 02 & 03 & 04 & 05 & 06 & 07 & 08 & 09 & 010 & 011 & UNIT CODES \\
\hline $\begin{array}{l}\text { Since last [day of } \\
\text { week today] did you } \\
\text { or others in your } \\
\text { household acquire } \\
\text { any [name of food } \\
\text { item]? }\end{array}$ & & & 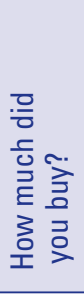 & & 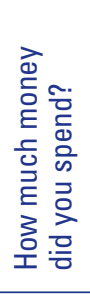 & 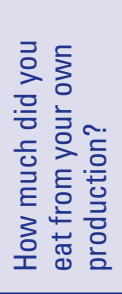 & & 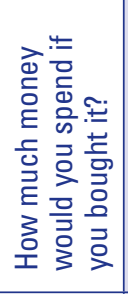 & 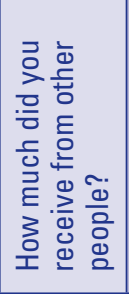 & & 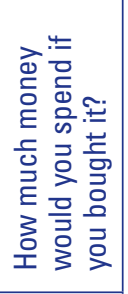 & $\begin{array}{l}\text { DEMONSTRATE } \\
\text { BOLDFACED CODES } \\
1 \text { KILOGRAM } \\
2 \text { GRAM } \\
3 \text { LITER } \\
4 \text { MILLILITER }\end{array}$ \\
\hline $\begin{array}{l}\text { GO THROUGH } \\
\text { ENTIRE LIST FIRST. } \\
\text { THEN GO TO 03-011 } \\
\text { FOR ITEMS WITH } \\
\text { “YES.” }\end{array}$ & $\begin{array}{ll}Y & 1 \\
N & 2\end{array}$ & $\begin{array}{l}\text { 㟔 } \\
\text { U } \\
\text { 岕 } \\
=\end{array}$ & 主 & 占 & $\begin{array}{c}\widehat{\underline{x}} \\
\underline{\cong}\end{array}$ & 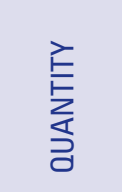 & 竔 & $\begin{array}{c}\widehat{\underline{x}} \\
\underline{\cong}\end{array}$ & 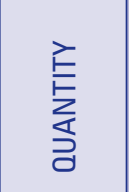 & 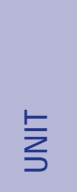 & 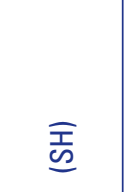 & $\begin{array}{l}5 \text { CENTIMETER } \\
6 \text { PACKET } \\
7 \text { LOAF }\end{array}$ \\
\hline \multicolumn{12}{|l|}{ Cereals } & 9 MARG TIN $1 \mathrm{KG}$ \\
\hline Maize, grain & & 101 & & & & & & & & & & 10 MARG. TIN, 1/2 KG \\
\hline Maize, flour & & 102 & & & & & & & & & & 11 CUP, $1 / 4$ LITER \\
\hline Rice, local, paddy & & 103 & & & & & & & & & & 12 SPOON \\
\hline Rice, local, husked & & 104 & & & & & & & & & & 13 SODA BOTTLE LID \\
\hline Rice, imported & & 105 & & & & & & & & & & 14 RICE SACK, 25 KG \\
\hline Millet & & 106 & & & & & & & & & & 15 OIL TIN, 20 LITER \\
\hline Sorghum & & 107 & & & & & & & & & & $\begin{array}{l}16 \text { SODA BOTTLE } \\
17 \text { BEER BOTTLE SMALL }\end{array}$ \\
\hline Bread & & 108 & & & & & & & & & & 18 BEER BOTTLE, LARGE \\
\hline Buns & & 109 & & & & & & & & & & 19 BUCKET \\
\hline Spaghetti or macaroni & & 110 & & & & & & & & & & 20 HEAP \\
\hline Breakfast cereal & & 111 & & & & & & & & & & $21 \mathrm{BUNCH}$ \\
\hline Cake & & 112 & & & & & & & & & & 22 OTHER (SPECIFY) \\
\hline Other & & 198 & & & & & & & & & & \\
\hline Other & & 199 & & & & & & & & & & \\
\hline
\end{tabular}


MODEL SURVEY MODULE ON ACQUISITION OF FOOD OVER THE PAST WEEK (continued)

\begin{tabular}{|c|c|c|c|c|c|c|c|c|c|c|c|c|}
\hline & 01 & 02 & 03 & 04 & 05 & 06 & 07 & 08 & 09 & $\mathbf{0 1 0}$ & 011 & UNIT CODES \\
\hline $\begin{array}{l}\text { Since last [day of } \\
\text { week today] did you } \\
\text { or others in your } \\
\text { household acquire } \\
\text { any [name of food } \\
\text { item]? }\end{array}$ & & & 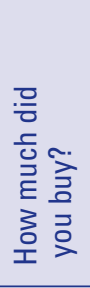 & & 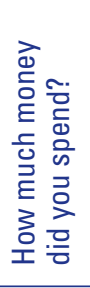 & 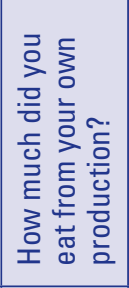 & & 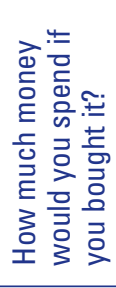 & 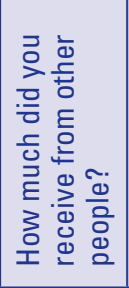 & & 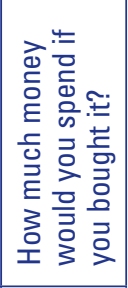 & $\begin{array}{l}\text { DEMONSTRATE } \\
\text { BOLDFACED CODES } \\
1 \text { KILOGRAM } \\
2 \text { GRAM } \\
3 \text { LITER } \\
4 \text { MILLILITER }\end{array}$ \\
\hline $\begin{array}{l}\text { GO THROUGH } \\
\text { ENTIRE LIST FIRST. } \\
\text { THEN GO TO 03-011 } \\
\text { FOR ITEMS WITH } \\
\text { "YES." }\end{array}$ & $\begin{array}{ll}Y & 1 \\
N & 2\end{array}$ & $\begin{array}{l}\text { 㟔 } \\
\text { ○ } \\
\sum \\
\text { 饪 }\end{array}$ & 导 & $\stackrel{\text { Ł }}{\leftrightarrows}$ & $\widehat{\widehat{I}}$ & 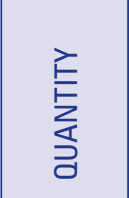 & 衣 & $\widehat{\widehat{I}}$ & 点 & ఏ & $\widehat{\widehat{્}}$ & $\begin{array}{l}5 \text { CENTIMETER } \\
6 \text { PACKET } \\
7 \text { LOAF }\end{array}$ \\
\hline $\begin{array}{l}\text { Roots, tubers, } \\
\text { and plantains }\end{array}$ & & & & & & & & & & & & $\begin{array}{l}8 \text { UNITIES } \\
9 \text { MARG. TIN, } 1 \text { KG }\end{array}$ \\
\hline Cassava, size $1(\mathrm{~cm})$ & & 201 & & & & & & & & & & 10 MARG. TIN, 12 KG \\
\hline Cassava, size $2(\mathrm{~cm})$ & & 202 & & & & & & & & & & $\begin{array}{l}11 \text { CUP, } 1 / 4 \text { LIIER } \\
12 \text { SPON }\end{array}$ \\
\hline Cassava, size $3(\mathrm{~cm})$ & & 203 & & & & & & & & & & 13 SODA BOTTLE LID \\
\hline Cassava, dried & & 204 & & & & & & & & & & 14 RICE SACK, 25 KG \\
\hline Cassava, flour & & 205 & & & & & & & & & & 15 OIL TIN, 20 LITER \\
\hline Potatoes & & 206 & & & & & & & & & & 16 SODA BOTTLE \\
\hline Sweet potatoes & & 207 & & & & & & & & & & 17 BEER BOTTLE, SMALL \\
\hline Yams & & 208 & & & & & & & & & & 19 BUCKET \\
\hline Plantain bananas & & 209 & & & & & & & & & & 20 HEAP \\
\hline Other & & 198 & & & & & & & & & & $21 \mathrm{BUNCH}$ \\
\hline Other & & 199 & & & & & & & & & & 22 OTHER (SPECIFY) \\
\hline
\end{tabular}

36 Measuring Food Security Using Household Expenditure Surveys 
MODEL SURVEY MODULE ON ACQUISITION OF FOOD OVER THE PAST WEEK (continued)

\begin{tabular}{|c|c|c|c|c|c|c|c|c|c|c|c|c|}
\hline & 01 & 02 & 03 & 04 & 05 & 06 & 07 & 08 & 09 & 010 & 011 & UNIT CODES \\
\hline $\begin{array}{l}\text { Since last [day of } \\
\text { week today] did you } \\
\text { or others in your } \\
\text { household acquire } \\
\text { any [name of food } \\
\text { item]? }\end{array}$ & & & 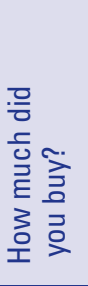 & & 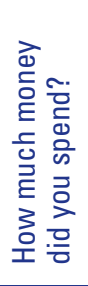 & 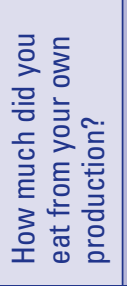 & & 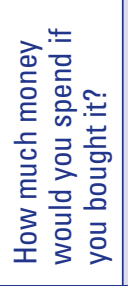 & 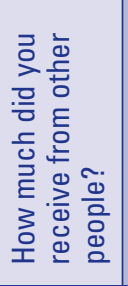 & & 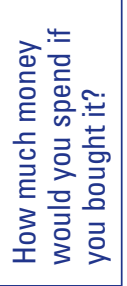 & $\begin{array}{l}\text { DEMONSTRATE } \\
\text { BOLDFACED CODES } \\
1 \text { KILOGRAM } \\
2 \text { GRAM } \\
3 \text { LITER } \\
4 \text { MILLILITER }\end{array}$ \\
\hline $\begin{array}{l}\text { GO THROUGH } \\
\text { ENTIRE LIST FIRST. } \\
\text { THEN GO TO 03-011 } \\
\text { FOR ITEMS WITH } \\
\text { "YES." }\end{array}$ & $\begin{array}{lll}\mathrm{Y} & 1 \\
\mathrm{~N} & 2\end{array}$ & $\begin{array}{l}\text { 㟧 } \\
\text { 嵌 }\end{array}$ & $\begin{array}{l}\text { 产 } \\
\text { 离 }\end{array}$ & 言 & 吉 & $\begin{array}{l}\text { 鹿 } \\
\text { 詹 }\end{array}$ & $\bar{\Xi}$ & 空 & $\begin{array}{l}\text { 恶 } \\
\text { 言 }\end{array}$ & 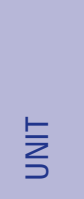 & 啭 & $\begin{array}{l}5 \text { CENTIMETER } \\
6 \text { PACKET } \\
7 \text { LOAF }\end{array}$ \\
\hline $\begin{array}{l}\text { Pulses, nuts, } \\
\text { and seeds }\end{array}$ & & & & & & & & & & & & $\begin{array}{l}8 \text { UNITIES } \\
9 \text { MARG. TIN, } 1 \text { KG }\end{array}$ \\
\hline Beans, dry & & 301 & & & & & & & & & & 10 MARG. TIN, 1/2 KG \\
\hline Peas, dry & & 302 & & & & & & & & & & 12 SPOON \\
\hline Lentils & & 303 & & & & & & & & & & 13 SODA BOTTLE LID \\
\hline Groundnuts, in shell & & 304 & & & & & & & & & & 14 RICE SACK, 25 KG \\
\hline Groundnuts, shelled & & 305 & & & & & & & & & & $\begin{array}{l}15 \text { OIL TIN, } 20 \text { LITER } \\
16 \text { SORA BOTTIF }\end{array}$ \\
\hline Coconuts, mature & & 306 & & & & & & & & & & 17 BEER BOTTLE, SMALL \\
\hline Coconuts, young & & 307 & & & & & & & & & & 18 BEER BOTTLE, LARGE \\
\hline Cashews & & 308 & & & & & & & & & & 19 BUCKET \\
\hline Sesame seeds & & 309 & & & & & & & & & & 20 HEAP \\
\hline Groundnut paste & & 310 & & & & & & & & & & 22 OTHER (SPECIFY) \\
\hline Baked beans, canned & & 311 & & & & & & & & & & \\
\hline Other & & 398 & & & & & & & & & & \\
\hline Other & & 399 & & & & & & & & & & \\
\hline
\end{tabular}


MODEL SURVEY MODULE ON ACQUISITION OF FOOD OVER THE PAST WEEK (continued)

\begin{tabular}{|c|c|c|c|c|c|c|c|c|c|c|c|c|}
\hline & 01 & 02 & 03 & 04 & 05 & 06 & 07 & 08 & 09 & 010 & 011 & UNIT CODES \\
\hline $\begin{array}{l}\text { Since last [day of } \\
\text { week today] did you } \\
\text { or others in your } \\
\text { household acquire } \\
\text { any [name of food } \\
\text { item]? }\end{array}$ & & & 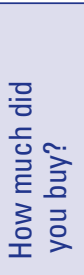 & & 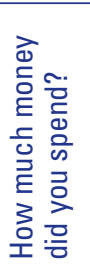 & 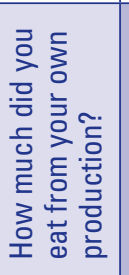 & & 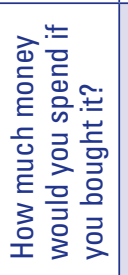 & 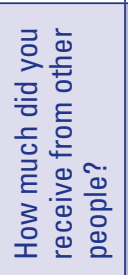 & & 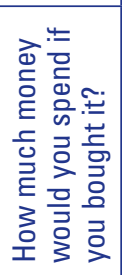 & $\begin{array}{l}\text { DEMONSTRATE } \\
\text { BOLDFACED CODES } \\
1 \text { KILOGRAM } \\
2 \text { GRAM } \\
3 \text { LITER }\end{array}$ \\
\hline $\begin{array}{l}\text { GO THROUGH } \\
\text { ENTIRE LIST FIRST. } \\
\text { THEN GO TO 03-011 } \\
\text { FOR ITEMS WITH } \\
\text { "YES." }\end{array}$ & $\begin{array}{ll}Y & 1 \\
N & 2\end{array}$ & $\begin{array}{l}\text { 㟔 } \\
\text { 仓 } \\
\text { 嵌 }\end{array}$ & 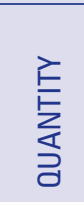 & $\stackrel{\text { Ł }}{\leftrightarrows}$ & 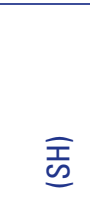 & 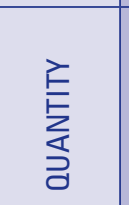 & 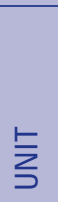 & $\widehat{\widehat{્}}$ & 点 & 亲 & $\widehat{\widehat{\underline{S}}}$ & $\begin{array}{l}4 \text { MILLILITER } \\
5 \text { CENTIMETER } \\
6 \text { PACKET } \\
7 \text { LOAF }\end{array}$ \\
\hline Vegetables & & & & & & & & & & & & 8 UNITIES \\
\hline Carrots & & 401 & & & & & & & & & & 9 MARG. TIN, 1 KG \\
\hline Onions & & 402 & & & & & & & & & & 10 MARG. TIN, 1/2 KG \\
\hline Garlic & & 403 & & & & & & & & & & 12 SPOON \\
\hline Okra & & 404 & & & & & & & & & & \\
\hline Cabbage & & 405 & & & & & & & & & & 14 RICE SACK, 25 KG \\
\hline Cassava leaves & & 406 & & & & & & & & & & 15 OIL TIN, 20 LITER \\
\hline Kale & & 407 & & & & & & & & & & 16 SODA BOTTLE \\
\hline Spinach & & 408 & & & & & & & & & & 17 BEER BOTTLE, SMALL \\
\hline Tomatoes & & 409 & & & & & & & & & & 18 BEER BOTTLE, LARGE \\
\hline Eggplant & & 410 & & & & & & & & & & 19 BUCKET \\
\hline Sweet corn & & 411 & & & & & & & & & & 20 HEAP \\
\hline Squash & & 412 & & & & & & & & & & $21 \mathrm{BUNCH}$ \\
\hline Beans, fresh & & 413 & & & & & & & & & & 22 OTHER (SPECIFY) \\
\hline Tomato paste, canned & & 414 & & & & & & & & & & \\
\hline Beans, fresh, canned & & 415 & & & & & & & & & & \\
\hline Sweet corn, canned & & 416 & & & & & & & & & & \\
\hline Other & & 498 & & & & & & & & & & \\
\hline Other & & 499 & & & & & & & & & & \\
\hline
\end{tabular}

38 Measuring Food Security Using Housebold Expenditure Surveys 
MODEL SURVEY MODULE ON ACQUISITION OF FOOD OVER THE PAST WEEK (continued)

\begin{tabular}{|c|c|c|c|c|c|c|c|c|c|c|c|c|}
\hline & 01 & 02 & 03 & 04 & 05 & 06 & 07 & 08 & 09 & 010 & 011 & \multirow{18}{*}{$\begin{array}{l}\text { UNIT CODES } \\
\text { DEMONSTRATE } \\
\text { BOLDFACED CODES } \\
1 \text { KILOGRAM } \\
2 \text { GRAM } \\
3 \text { LITER } \\
4 \text { MILLILITER } \\
5 \text { CENTIMETER } \\
6 \text { PACKET } \\
7 \text { LOAF } \\
8 \text { UNITIES } \\
9 \text { MARG. TIN, 1 KG } \\
10 \text { MARG. TIN, 1/2 KG } \\
11 \text { CUP, 1/4 LITER } \\
12 \text { SPOON } \\
13 \text { SODA BOTTLE LID } \\
14 \text { RICE SACK, 25 KG } \\
15 \text { OIL TIN, 20 LITER } \\
16 \text { SODA BOTTLE } \\
17 \text { BEER BOTTLE, SMALL } \\
18 \text { BEER BOTTLE, LARGE } \\
19 \text { BUCKET } \\
20 \text { HEAP } \\
21 \text { BUNCH } \\
22 \text { OTHER (SPECIFY) } \\
\end{array}$} \\
\hline $\begin{array}{l}\text { Since last [day of } \\
\text { week today] did you } \\
\text { or others in your } \\
\text { household acquire } \\
\text { any [name of food } \\
\text { item]? }\end{array}$ & & & 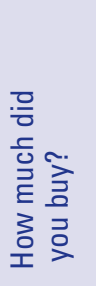 & & 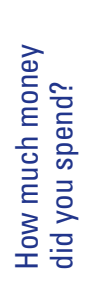 & 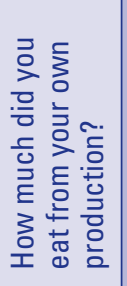 & & 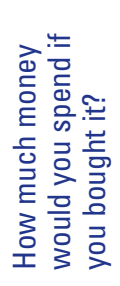 & 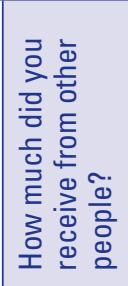 & & 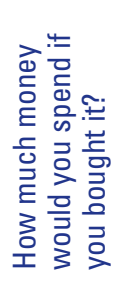 & \\
\hline $\begin{array}{l}\text { GO THROUGH ENTIRE } \\
\text { LIST FIRST. } \\
\text { THEN GO TO 03-011 } \\
\text { FOR ITEMS WITH } \\
\text { "YES." }\end{array}$ & $\begin{array}{ll}Y & 1 \\
N & 2\end{array}$ & $\begin{array}{l}\text { 㟧 } \\
\text { 仓 } \\
\sum_{\text {․․․ }}\end{array}$ & $\begin{array}{l}\text { 䘚 } \\
\text { 㚆 }\end{array}$ & 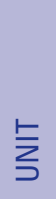 & $\widehat{\overline{\mathbf{I}}}$ & $\begin{array}{l}\text { 差 } \\
\text { 京 }\end{array}$ & 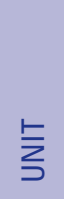 & 焉 & 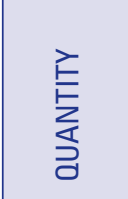 & 言 & 㞼 & \\
\hline Fruits & & & & & & & & & & & & \\
\hline Bananas, sweet & & 501 & & & & & & & & & & \\
\hline Oranges & & 502 & & & & & & & & & & \\
\hline Grapefruit & & 503 & & & & & & & & & & \\
\hline Lemons & & 504 & & & & & & & & & & \\
\hline Avocadoes & & 505 & & & & & & & & & & \\
\hline Guavas & & 506 & & & & & & & & & & \\
\hline Mangoes & & 507 & & & & & & & & & & \\
\hline Papayas & & 508 & & & & & & & & & & \\
\hline Passion fruit & & 509 & & & & & & & & & & \\
\hline Watermelons & & 510 & & & & & & & & & & \\
\hline Peaches, canned & & 511 & & & & & & & & & & \\
\hline Pears, canned & & 512 & & & & & & & & & & \\
\hline Other & & 598 & & & & & & & & & & \\
\hline Other & & 599 & & & & & & & & & & \\
\hline
\end{tabular}


MODEL SURVEY MODULE ON ACQUISITION OF FOOD OVER THE PAST WEEK (continued)

\begin{tabular}{|c|c|c|c|c|c|c|c|c|c|c|c|c|}
\hline & 01 & 02 & 03 & 04 & 05 & 06 & 07 & 08 & 09 & 010 & 011 & UNIT CODES \\
\hline $\begin{array}{l}\text { Since last [day of } \\
\text { week today] did you } \\
\text { or others in your } \\
\text { household acquire } \\
\text { any [name of food } \\
\text { item]? }\end{array}$ & & & 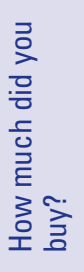 & & 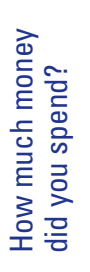 & 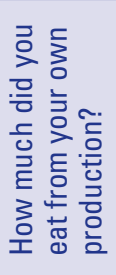 & & 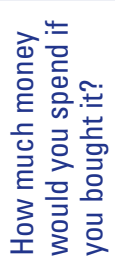 & 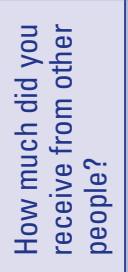 & & 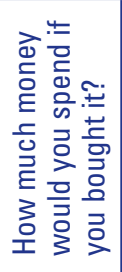 & $\begin{array}{l}\text { DEMONSTRATE } \\
\text { BOLDFACED CODES } \\
1 \text { KILOGRAM } \\
2 \text { GRAM } \\
3 \text { LITER }\end{array}$ \\
\hline $\begin{array}{l}\text { GO THROUGH } \\
\text { ENTIRE LIST FIRST. } \\
\text { THEN GO TO O3-011 } \\
\text { FOR ITEMS WITH } \\
\text { "YES." }\end{array}$ & $\begin{array}{ll}Y & 1 \\
N & 2\end{array}$ & $\begin{array}{l}\text { 㟔 } \\
\text { 品 } \\
\sum_{\text {嵌 }}\end{array}$ & $\begin{array}{l}\text { 点 } \\
\text { 交 }\end{array}$ & 歺 & 㒰 & 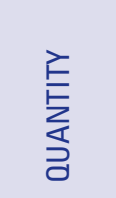 & 歺 & 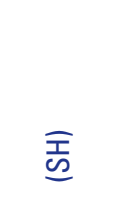 & 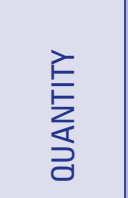 & $\sum_{う}$ & $\widehat{\widehat{\underline{\Xi}}}$ & $\begin{array}{l}5 \text { CENTIMETER } \\
6 \text { PACKET } \\
7 \text { LOAF }\end{array}$ \\
\hline $\begin{array}{l}\text { Meat, poultry, } \\
\text { and insects }\end{array}$ & & & & & & & & & & & & $\begin{array}{l}8 \text { UNITIES } \\
9 \text { MARG. TIN, } 1 \text { KG }\end{array}$ \\
\hline Beef, with bones & & 601 & & & & & & & & & & 10 MARG. TIN, 1/2 KG \\
\hline Beef, without bones & & 602 & & & & & & & & & & 11 CUP, 1/4 LITER \\
\hline Pork, with bones & & 603 & & & & & & & & & & 12 SPOON \\
\hline Pork, without bones & & 604 & & & & & & & & & & 13 SODA BOTTLE LID \\
\hline Goat, with bones & & 605 & & & & & & & & & & 14 RICE SACK, 25 KG \\
\hline Goat, without bones & & 606 & & & & & & & & & & 15 OIL TIN, 20 LITER \\
\hline Wild meat & & 607 & & & & & & & & & & 16 SODA BOTTLE \\
\hline Rabbit & & 608 & & & & & & & & & & 17 BEER BOTTLE, SMALL \\
\hline Insects & & 609 & & & & & & & & & & 18 BEER BOTTLE, LARGE \\
\hline Liver & & 610 & & & & & & & & & & 19 BUCKET \\
\hline Kidneys & & 611 & & & & & & & & & & 20 HEAP \\
\hline Heart & & 612 & & & & & & & & & & $21 \mathrm{BUNCH}$ \\
\hline Chicken & & 613 & & & & & & & & & & 22 OTHER (SPECIFY) \\
\hline Duck & & 614 & & & & & & & & & & \\
\hline Turkey & & 615 & & & & & & & & & & \\
\hline Corned beef, canned & & 616 & & & & & & & & & & \\
\hline Pork, sausages & & 617 & & & & & & & & & & \\
\hline Other & & 698 & & & & & & & & & & \\
\hline Other & & 699 & & & & & & & & & & \\
\hline
\end{tabular}

40 Measuring Food Security Using Household Expenditure Surveys 
MODEL SURVEY MODULE ON ACQUISITION OF FOOD OVER THE PAST WEEK (continued)

\begin{tabular}{|c|c|c|c|c|c|c|c|c|c|c|c|c|}
\hline & 01 & 02 & 03 & 04 & 05 & 06 & 07 & 08 & 09 & 010 & 011 & \multirow{18}{*}{$\begin{array}{l}\text { UNIT CODES } \\
\text { DEMONSTRATE } \\
\text { BOLDFACED CODES } \\
1 \text { KILOGRAM } \\
2 \text { GRAM } \\
3 \text { LITER } \\
4 \text { MILLILITER } \\
5 \text { CENTIMETER } \\
6 \text { PACKET } \\
7 \text { LOAF } \\
8 \text { UNITIES } \\
9 \text { MARG. TIN, } 1 \text { KG } \\
10 \text { MARG. TIN, } 1 / 2 \text { KG } \\
11 \text { CUP, 1/4 LITER } \\
12 \text { SPOON } \\
13 \text { SODA BOTTLE LID } \\
14 \text { RICE SACK, } 25 \text { KG } \\
15 \text { OIL TIN, 20 LITER } \\
16 \text { SODA BOTTLE } \\
17 \text { BEER BOTTLE, SMALL } \\
18 \text { BEER BOTTLE, LARGE } \\
19 \text { BUCKET } \\
20 \text { HEAP } \\
21 \text { BUNCH } \\
22 \text { OTHER (SPECIFY) } \\
\end{array}$} \\
\hline $\begin{array}{l}\text { Since last [day of } \\
\text { week today] did you } \\
\text { or others in your } \\
\text { household acquire } \\
\text { any [name of food } \\
\text { item]? }\end{array}$ & & & 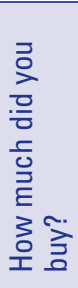 & & 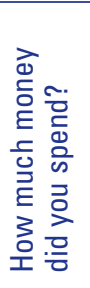 & 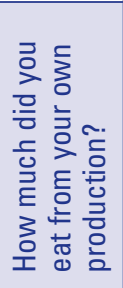 & & 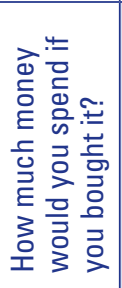 & 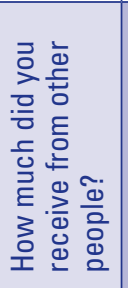 & & 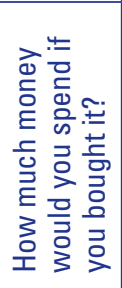 & \\
\hline $\begin{array}{l}\text { GO THROUGH } \\
\text { ENTIRE LIST FIRST. } \\
\text { THEN GO TO 03-011 } \\
\text { FOR ITEMS WITH } \\
\text { "YES." }\end{array}$ & $\begin{array}{ll}Y & 1 \\
N & 2\end{array}$ & $\begin{array}{l}\text { 㟧 } \\
\text { 仓 } \\
\sum_{\text {殅 }}\end{array}$ & 忘 & ఏ & 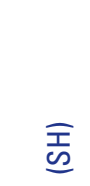 & 铕 & 衣 & $\widehat{\widehat{I}}$ & 悥 & 衣 & $\widehat{\underline{\underline{N}}}$ & \\
\hline \multicolumn{12}{|l|}{ Fish and seafood } & \\
\hline $\begin{array}{l}\text { Fish, fresh, whole, } \\
\text { size } 1 \text { (cm) }\end{array}$ & & 701 & & & & & & & & & & \\
\hline $\begin{array}{l}\text { Fish, fresh, whole, } \\
\text { size } 2 \text { (cm) }\end{array}$ & & 702 & & & & & & & & & & \\
\hline $\begin{array}{l}\text { Fish, fresh, whole, } \\
\text { size } 3(\mathrm{~cm})\end{array}$ & & 703 & & & & & & & & & & \\
\hline $\begin{array}{l}\text { Fish, fresh, whole, } \\
\text { dried (cm) }\end{array}$ & & 704 & & & & & & & & & & \\
\hline Fish, fresh, fillet (cm) & & 705 & & & & & & & & & & \\
\hline Fish, dry, whole (cm) & & 706 & & & & & & & & & & \\
\hline Fish, dry, fillet (cm) & & 707 & & & & & & & & & & \\
\hline Fish, tuna, canned & & 708 & & & & & & & & & & \\
\hline Fish, sardines, canned & & 709 & & & & & & & & & & \\
\hline Shrimp & & 710 & & & & & & & & & & \\
\hline Crab & & 711 & & & & & & & & & & \\
\hline Lobster & & 712 & & & & & & & & & & \\
\hline Other & & 798 & & & & & & & & & & \\
\hline Other & & 799 & & & & & & & & & & \\
\hline
\end{tabular}


MODEL SURVEY MODULE ON ACQUISITION OF FOOD OVER THE PAST WEEK (continued)

\begin{tabular}{|c|c|c|c|c|c|c|c|c|c|c|c|c|}
\hline & 01 & 02 & 03 & 04 & 05 & 06 & 07 & 08 & 09 & 010 & 011 & \multirow{4}{*}{$\begin{array}{l}\text { UNIT CODES } \\
\text { DEMONSTRATE } \\
\text { BOLDFACED CODES } \\
1 \text { KILOGRAM } \\
2 \text { GRAM } \\
3 \text { LITER } \\
4 \text { MILLILITER } \\
5 \text { CENTIMETER } \\
6 \text { PACKET } \\
7 \text { LOAF } \\
8 \text { UNITIES } \\
9 \text { MARG. TIN, } 1 \text { KG }\end{array}$} \\
\hline $\begin{array}{l}\text { Since last [day of } \\
\text { week today] did you } \\
\text { or others in your } \\
\text { household acquire } \\
\text { any [name of food } \\
\text { item]? }\end{array}$ & & & 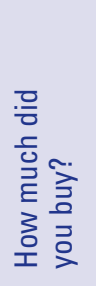 & & 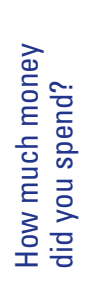 & 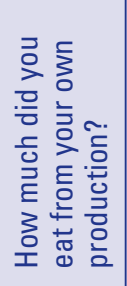 & & 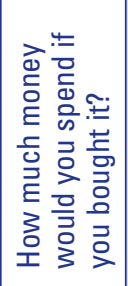 & 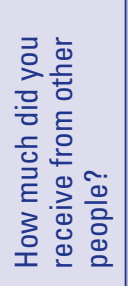 & & 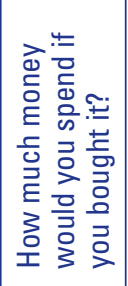 & \\
\hline $\begin{array}{l}\text { GO THROUGH } \\
\text { ENTIRE LIST FIRST. } \\
\text { THEN GO TO O3-011 } \\
\text { FOR ITEMS WITH } \\
\text { "YES." }\end{array}$ & $\begin{array}{ll}\text { Y } & 1 \\
\text { N } & 2\end{array}$ & 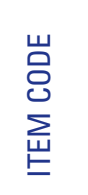 & 意 & $\overline{\grave{\zeta}}$ & 㤩 & 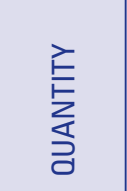 & $\bar{\Xi}$ & 丞 & 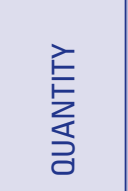 & 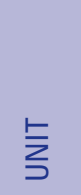 & 嵒 & \\
\hline Oils and fats & & & & & & & & & & & & \\
\hline Peanut oil & & 1001 & & & & & & & & & & 10 MARG. TIN, 1/2 KG \\
\hline Coconut oil & & 1002 & & & & & & & & & & 11 CUP, $1 / 4$ LITER \\
\hline Palm oil & & 1003 & & & & & & & & & & 12 SPOON \\
\hline Margarine & & 1004 & & & & & & & & & & 14 RICE SACK, 25 KG \\
\hline Shea butter & & 1005 & & & & & & & & & & 15 OIL TIN, 20 LITER \\
\hline Animal fat & & 1006 & & & & & & & & & & 16 SODA BOTTLE \\
\hline Other & & 1098 & & & & & & & & & & 17 BEER BOTTLE, SMALL \\
\hline Other & & 1099 & & & & & & & & & & 19 BUCKET \\
\hline Beverages & & & & & & & & & & & & 20 HEAP \\
\hline Beer, local & & 1101 & & & & & & & & & & $21 \mathrm{BUNCH}$ \\
\hline Beer, imported & & 1102 & & & & & & & & & & 22 OTHER (SPECIFY) \\
\hline Wine & & 1103 & & & & & & & & & & \\
\hline Distilled alcohol, local & & 1104 & & & & & & & & & & \\
\hline $\begin{array}{l}\text { Distilled alcohol, } \\
\text { imported }\end{array}$ & & 1105 & & & & & & & & & & \\
\hline Soft drinks & & 1106 & & & & & & & & & & \\
\hline
\end{tabular}


MODEL SURVEY MODULE ON ACQUISITION OF FOOD OVER THE PAST WEEK (continued)

\begin{tabular}{|c|c|c|c|c|c|c|c|c|c|c|c|c|}
\hline & 01 & 02 & 03 & 04 & 05 & 06 & 07 & 08 & 09 & 010 & 011 & UNIT CODES \\
\hline $\begin{array}{l}\text { Since last [day of } \\
\text { week today] did you } \\
\text { or others in your } \\
\text { household acquire } \\
\text { any [name of food } \\
\text { item]? }\end{array}$ & & & 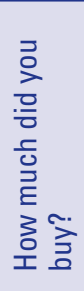 & & 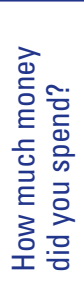 & 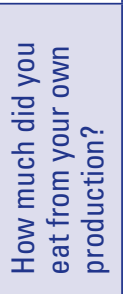 & & 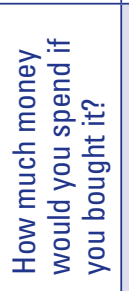 & 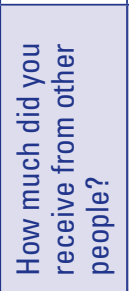 & & 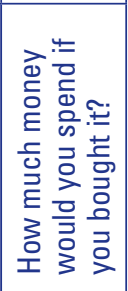 & $\begin{array}{l}\text { DEMONSTRATE } \\
\text { BOLDFACED CODES } \\
1 \text { KILOGRAM } \\
2 \text { GRAM } \\
3 \text { LITER } \\
4 \text { MUMUITER }\end{array}$ \\
\hline $\begin{array}{l}\text { GO THROUGH } \\
\text { ENTIRE LIST FIRST. } \\
\text { THEN GO TO Q3-011 } \\
\text { FOR ITEMS WITH } \\
\text { "YES." }\end{array}$ & $\begin{array}{ll}Y & 1 \\
N & 2\end{array}$ & $\begin{array}{l}\text { 㟔 } \\
\text { O } \\
\sum_{\text {嵌 }}\end{array}$ & 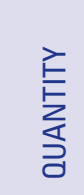 & 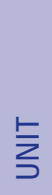 & 嵒 & 导 & $\stackrel{\sqsubseteq}{\vdots}$ & $\widehat{\underline{\underline{S}}}$ & 导 & 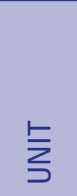 & $\widehat{\underline{\underline{D}}}$ & $\begin{array}{l}5 \text { CENTIMETER } \\
6 \text { PACKET } \\
7 \text { LOAF }\end{array}$ \\
\hline Beverages (continued) & & & & & & & & & & & & 8 UNITIES \\
\hline Fruit juices & & 1107 & & & & & & & & & & 10 MARG. TIN, $1 / 2$ KG \\
\hline Coffee & & 1108 & & & & & & & & & & 11 CUP, $1 / 4$ LITER \\
\hline Tea & & 1109 & & & & & & & & & & 12 SPOON \\
\hline Other & & 1198 & & & & & & & & & & 13 SODA BOTTLE LID \\
\hline Other & & 1199 & & & & & & & & & & 14 RICE SACK, 25 KG \\
\hline Miscellaneous & & & & & & & & & & & & 16 SODA BOTTLE \\
\hline Sugar & & 1201 & & & & & & & & & & 17 BEER BOTTLE, SMALL \\
\hline Salt & & 1202 & & & & & & & & & & 18 BEER BOTTLE, LARGE \\
\hline Pepper & & 1203 & & & & & & & & & & 19 BUCKET \\
\hline Curry powder & & 1204 & & & & & & & & & & 20 HEAP \\
\hline Other spices & & 1205 & & & & & & & & & & 22 OTHER (SPECIFY) \\
\hline Honey & & 1206 & & & & & & & & & & \\
\hline Jam & & 1207 & & & & & & & & & & \\
\hline Candy & & 1208 & & & & & & & & & & \\
\hline Chocolate & & 1209 & & & & & & & & & & \\
\hline
\end{tabular}

44 Measuring Food Security Using Household Expenditure Surveys 
MODEL SURVEY MODULE ON ACQUISITION OF FOOD OVER THE PAST WEEK (continued)

\begin{tabular}{|c|c|c|c|c|c|c|c|c|c|c|c|c|}
\hline & 01 & 02 & 03 & 04 & 05 & 06 & 07 & 08 & 09 & 010 & 011 & UNIT CODES \\
\hline $\begin{array}{l}\text { Since last [day of } \\
\text { week today] did you } \\
\text { or others in your } \\
\text { household acquire } \\
\text { any [name of food } \\
\text { item]? }\end{array}$ & & & 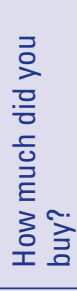 & & 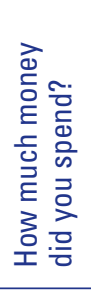 & 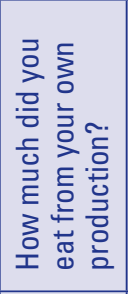 & & 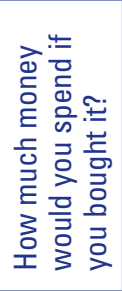 & 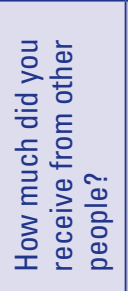 & & 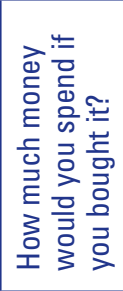 & $\begin{array}{l}\text { DEMONSTRATE } \\
\text { BOLDFACED CODES } \\
1 \text { KILOGRAM } \\
2 \text { GRAM } \\
3 \text { LITER } \\
4 \text { MILLILITER }\end{array}$ \\
\hline $\begin{array}{l}\text { GO THROUGH } \\
\text { ENTIRE LIST FIRST. } \\
\text { THEN GO TO 03-011 } \\
\text { FOR ITEMS WITH } \\
\text { "YES." }\end{array}$ & $\begin{array}{ll}Y & 1 \\
N & 2\end{array}$ & $\begin{array}{l}\text { 㟔 } \\
\text { 品 } \\
\text { 嵌 }\end{array}$ & $\begin{array}{l}\text { 严 } \\
\text { 志 }\end{array}$ & 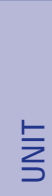 & $\widehat{\underline{\mathbf{I}}}$ & $\begin{array}{l}\text { 㺯 } \\
\text { 志 }\end{array}$ & 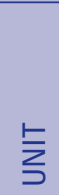 & 퐁 & $\begin{array}{l}\text { 点 } \\
\text { 志 }\end{array}$ & 衣 & $\widehat{\underline{\underline{D}}}$ & $\begin{array}{l}5 \text { CENTIMETER } \\
6 \text { PACKET } \\
7 \text { LOAF }\end{array}$ \\
\hline $\begin{array}{l}\text { Miscellaneous } \\
\text { (continued) }\end{array}$ & & & & & & & & & & & & $\begin{array}{l}8 \text { UNITIES } \\
9 \text { MARG. TIN, } 1 \text { KG }\end{array}$ \\
\hline Chewing gum & & 1210 & & & & & & & & & & 10 MARG. TIN, $1 / 2$ KG \\
\hline Vinegar & & 1211 & & & & & & & & & & 11 CUP, $1 / 4$ LITER \\
\hline Other & & 1298 & & & & & & & & & & 12 SPOON \\
\hline Other & & 1299 & & & & & & & & & & 14 RICE SACK, 25 KG \\
\hline $\begin{array}{l}\text { Foods consumed } \\
\text { outside of home }\end{array}$ & & & & & & & & & & & & 15 OIL TIN, 20 LITER \\
\hline Dish or food 1 & & 1301 & & & & & & & & & & 17 BEFR BOTTIF SMAN \\
\hline Dish or food 2 & & 1302 & & & & & & & & & & 18 BEER BOTTLE, LARGE \\
\hline Dish or food 3 & & 1303 & & & & & & & & & & 19 BUCKET \\
\hline Dish or food 4 & & 1304 & & & & & & & & & & 20 HEAP \\
\hline Dish or food 5 & & 1305 & & & & & & & & & & $21 \mathrm{BUNCH}$ \\
\hline Dish or food 6 & & 1306 & & & & & & & & & & 22 OTHER (SPECIFY) \\
\hline Dish or food 7 & & 1307 & & & & & & & & & & \\
\hline Other & & 1398 & & & & & & & & & & \\
\hline Other & & 1399 & & & & & & & & & & \\
\hline
\end{tabular}

Note: Interviewer instructions are written in uppercase letters, while text to be read to respondents is written in lowercase letters. 


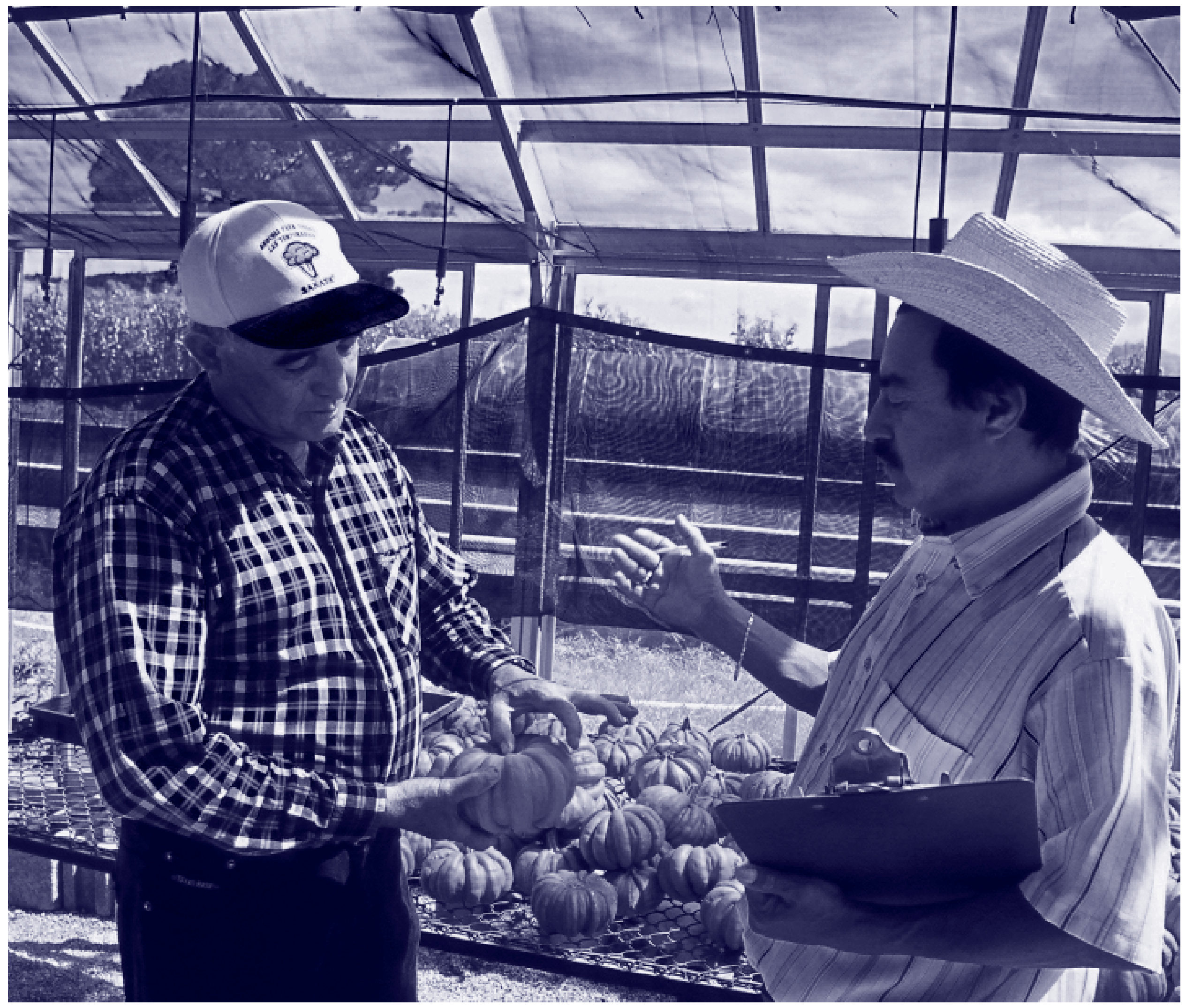




\section{Gathering Data for Calculating Metric Weights of Foods and Their Energy Content}

$\mathrm{T}$ 0 calculate most of the food security indicators, the food data collected from households must be converted from their raw form into metric weights of foods during the data processing stage. To do so, metric weight conversion factors for each food acquired by households and the unit of measure used for reporting must be collected, either in local markets or from secondary sources. If only expenditure data are collected from households, metric prices must be collected for each food.

This data collection can be done before, during, or after conducting the household surveys. The advantage of doing it before is that in some situations the process helps to determine whether a unit of measure is appropriate or precise enough. The advantage of doing it during data collection is that the enumerators are located close to where households obtain food and will be able to collect locationspecific data for units of measure that vary in size geographically. The advantage of doing it after is that, in cases in which households are allowed to choose a unit of measure, it will then be known which foods and which units of measure require conversions, and time will not be wasted gathering unneeded information. A disadvantage is that some foods may no longer be available due to seasonal fluctuations.

For many of the methods discussed in the following sections, weighing of food must take place. Past experience shows that in order to correctly weigh foods, those responsible for the task must be well trained and given ample opportunity to practice. It is very important that they be able to read scale output correctly and understand the concept of units of measure. Further, it is crucial that quality scales are used and that they are standardized (checked to make sure they are giving correct weights) periodically.

Gibson and Ferguson (1999) suggest some dietary scales (their Box 4.7, p. 41). Errors in reading scale output are reduced when digital scales are used, which is becoming more affordable.

\section{METRIC WEIGHTS OF FOODS MEASURED IN NONSTANDARD UNITS}

Foods are often reported in nonstandard units of measure when the "local unit of measure" and "count" methods are used for data collection. As noted in Chapter 3, the units may be of either fixed or variable size across households in a population. The methods of estimating metric weights of foods in the units of measure differ for these two types.

\section{Units of Measure of Fixed Size}

Because metric weights of foods reported in these units of measure (for example, 1/2-liter margarine tin) do not vary across households, only one weight is needed for each food in each unit of measure. The basic method is to weigh each food in each unit of measure for which a weight is needed using a scale. However, 
for some foods, time can be saved by using a reference food (for example, rice) and unit of measure to impute weights of other foods, as follows:

1. Weigh the reference food in the reference unit of measure.

2. Weigh the reference food in all other units of measure.

3. Weigh the rest of the foods in the reference unit of measure.

4. Calculate the ratio of the weight of the reference food to each other food using the weights in the reference unit of measure.

5. Use the ratios to impute the weights of all foods in all units of measure for which they are needed.

This method is best applied to foods that have small individual pieces that leave little open space between the pieces (for example, grains, flours, and liquids). It is not suited for roots and tubers and for most fruits and vegetables. Appendix 3 contains data on the weights of some foods in a standard-sized cup that can also be used for imputing weights.

Some tips follow.

- If there is a specific food associated with a unit of measure of known weight (for example, rice in a 25-kilogram rice bag) and the relative weight of foods compared with the original food is known in advance, no actual weighing of foods is needed for this unit of measure.

- If one type of container has several known sizes (for example, 1/2-, 1-, and 5-kilogram margarine tins), metric weights of foods in all sizes can be calculated if the weight is known in only one of them.

- In some cases foods are sold in cans with a specific metric weight clearly demarcated on them. In this case, the weight on the can should be used except in cases in which the food is in water, in which case the weight of the water must be estimated and subtracted from the weight on the can.

- In some areas sacks and tins are commonly overfilled. It is important to make sure that such "heaping" is taken into account when establishing metric weights of foods in various-sized containers by visiting vendors and determining the most common heaped amounts for specific foods and containers.

\section{Units of Measure of Variable Size}

Metric weights of foods reported in these types of unit of measure (for example, unities, heaps, bunches, and bowls) vary across households. Although it is infeasible to collect them for each household, they can be collected in communities or broader geographical areas in which households are located. The weights can be obtained by purchasing or "borrowing" a sample of food in the units of measure from either a vendor or a household and weighing it using a scale. Depending on the variability in weights, it is best to use the mean weight taken from multiple samples (three to five) as the conversion factor.

Note that where weighing is not possible, a rough estimate of the metric weight of a food in a local unit of measure of variable size can be obtained using the quantity and expenditure data reported by households if at least some households report in a metric unit. The method for doing so is as follows:

1. Calculate the mean of the unit values (expenditure divided by quantity) reported for the metric unit (for example, kilograms) 
across households. ${ }^{13}$ This gives an estimated price per kilogram.

2. Calculate the mean of the unit values reported for the local unit across households reporting in that unit (for example, heap), which gives an estimated price per heap. The ratio of the price per heap to the price per kilogram gives the kilogram equivalent of one heap. For example, if the mean unit value of a heap is 10 pesos and the mean unit value of a kilogram is 50 pesos, the estimated weight of one heap is one-fifth of a kilogram, or 200 grams.

In the case of the count method, the weights of foods can be looked up in some food composition tables. As a last resort, the United States food composition tables (USDA 2003), which give the "small," "medium," and "large" weights of some foods (for example, fruits and vegetables), can be used for foods that are known not to vary greatly in size across countries.

\section{METRIC WEIGHTS OF FOODS MEASURED IN VOLUMETRIC UNITS, IN LINEAR DIMENSIONS, AND USING FOOD MODELS}

Techniques for determining the metric weights of foods measured in volumetric units, linear dimensions, and using food models have been developed by Gibson and Ferguson (1999) and Swindale and Ohri-Vachaspati (2004).
Volumetric equivalents. The recorded metric volume of the food item can be converted to a metric weight equivalent using conversion factors calculated following one of three methods:

1. For beverages and other liquid foods: look up the millilitersto-grams conversion factor for each food in tables of "specific gravities." Specific gravities for foods commonly consumed in developing countries are given in Appendix 4.

2. For solid foods, look up the weight of the food in a standardsized container (for example, a 237-milliliter cup), which is reported in some food composition tables (for example, Siong et al. 1997, Dignan et al. 2004) and publications from food consumption surveys (for example, Dop et al. 2003). The weight of the food in the container divided by the volume in milliliters gives the milliliters-to-grams conversion factor.

3. Use a scale to weigh several samples of the food contained in a container of a specific volume. The milliliters-to-grams conversion factor is the weight of the food divided by the volume of the container.

Once a conversion factor for a reference food (for example, rice) has been calculated, the ratios of the weight of the food to the weights of other foods can be used to estimate conversion factors for the other foods. Weights for calculating these ratios for some common foods can be found in Appendix 3.

Linear dimensions. To estimate a centimeters-to-grams conversion factor, Gibson and Ferguson (1999) recommend following these steps:

${ }^{13}$ It is always useful to compare the mean to the median to make sure that the mean is not being driven by outliers. 
1. Cut the food item into pieces of various lengths (for example, 10 pieces).

2. Measure the length of each piece using a ruler; record in centimeters.

3. Weigh each piece using a scale; record in grams.

4. Divide the weight of each piece by its length, and calculate the centimeters-to-grams conversion factor.

Food models. Use a scale to weigh several samples of the food with the same dimensions as the model. Take the mean weight of the samples.

\section{PROPORTIONATE WEIGHTS OF INGREDIENTS IN PREPARED DISHES CONSUMED OUTSIDE THE HOME}

The proportionate weights of the raw ingredients in a prepared dish can be estimated in the following two steps (adapted from method in Gibson and Ferguson 1999).

1. Obtain the weight and price per gram of a serving of the dish as sold by vendors.

- Purchase a usual portion of the dish from several vendors, preferably three, in the study area, and weigh it (if the sizes and costs of dishes vary greatly from location to location within the survey area, the process will need to be repeated for multiple locations)

- Record the price of one portion.

- Calculate the average price per gram of the dish across the vendors
2. Calculate the proportionate weight of the raw ingredients in the dish. The respondent can be either a restaurant chef or a local person who regularly prepares the dish.

Direct observation:

- Have the respondent cook the dish. Weigh the raw ingredients used before cooking.

- Weigh the cooked dish.

- Divide the weight of each raw ingredient by the total weight of the cooked dish.

Recall:

- Ask the respondent what ingredients are used to cook the dish.

- Ask him or her to estimate the amounts used the last time the dish was cooked (using the methods for food data collection listed in Box 1).

- Convert the amounts to weights in grams.

- Ask the respondent to estimate the number of portions served to customers in the final cooked amount.

- Calculate the weight of the total cooked amount using the portion weight collected in Step 1.

- Divide the weight of each raw ingredient by the total weight of the cooked dish.

\section{METRIC FOOD PRICES}

Metric prices must be collected or derived for those foods for which the "monetary value" method of data collection is used. ${ }^{14}$ There are three main methods for obtaining price data: market price surveys, price opinions, and estimation of prices using unit values.

${ }^{14}$ The sources for this section are Deaton and Grosh (2000), Frankenberg (2000), ILO (2004), and Ghana Statistical Service (no date). 


\section{Market Price Surveys}

Market price surveys are considered the "gold standard" method of obtaining price data. ${ }^{15}$ Here food vendors located near the community in which households are being surveyed and from which households typically purchase foods are the intended informants. The vendors can be selected either by asking households during the household interviews about the markets and stores most frequently visited or by determining the market or store closest to the community. Note that in some countries the market or store that is the closest may be hard to determine. In urban areas, people may buy goods far away from where they live. In general, there is no guarantee that the prices in any given "local" market are the prices actually paid by households in the survey.

The price questionnaire should contain sufficient space for enumerators to record three key pieces of information: (1) the unit of measure being reported (if not prespecified), (2) the price per unit, and, where necessary, (3) the weight in metric units of the amount purchased from each vendor. Whenever possible, prices should be recorded in metric units. When foods are sold in local units, enumerators must weigh foods in the local unit and record the amount in metric units. ${ }^{16}$ In some areas vendors add a small amount to the quantity sold. If this is the practice, this amount should be weighed as well. It is important to use the unit of measure in which a food is normally purchased. For example, if the food is purchased in a tin by households but it is also possible to buy it in a 50-kilogram sack (a bulk purchase), it is best to obtain the price for the tin, which may differ.

The common practice is to collect prices from at least three vendors. Note, however, that for foods that are known in advance to have fixed prices, there is no need to collect from more than one vendor. For those whose prices vary from day to day and vendor to vendor, it is especially important to collect from multiple vendors and, if possible, make visits on separate days.

Sometimes enumerators will need to collect the price of a substitute item for one that is not available at the time of the data collection. In this case, the substitute should be the nearest equivalent product. Enough space needs to be given on the questionnaire to write in a detailed description of the substitute item.

Enumerators should try to replicate as much as possible the buying habits of households - including bargaining down the prices. They should take the opportunity to observe transaction prices for genuine sales and be able to recognize "deceptive" prices. The choice of whether to ask vendors how much buyers usually pay or to try to replicate transactions by purchasing foods will depend on the particular setting. In some situations it is difficult for enumerators to replicate the kinds of transactions that locals engage in. Haggling is often an important factor in defining prices paid. Vendors may quote different prices to enumerators than to longstanding or regular customers. In these situations households may be a better source of information than vendors.

In general, the prices should be collected as close as possible to the time of the household survey (within a month). In some areas very specific timing of price data collection is important, because it may affect the representativeness of the price. For example, in some Middle Eastern countries, prices vary by the time of day and week. Also note that in some countries the market is held on one day of the week. This should be kept in mind when scheduling a market price survey.

The following is a model price questionnaire.

15 The sources for this section are Deaton and Grosh (2000), Frankenberg (2000), ILO (2004), and Ghana Statistical Service (no date).

${ }^{16}$ Food weighing requires careful training of enumerators and quality scales, as mentioned in the introduction to this chapter. 
MODEL SURVEY MODULE ON FOOD PRICES

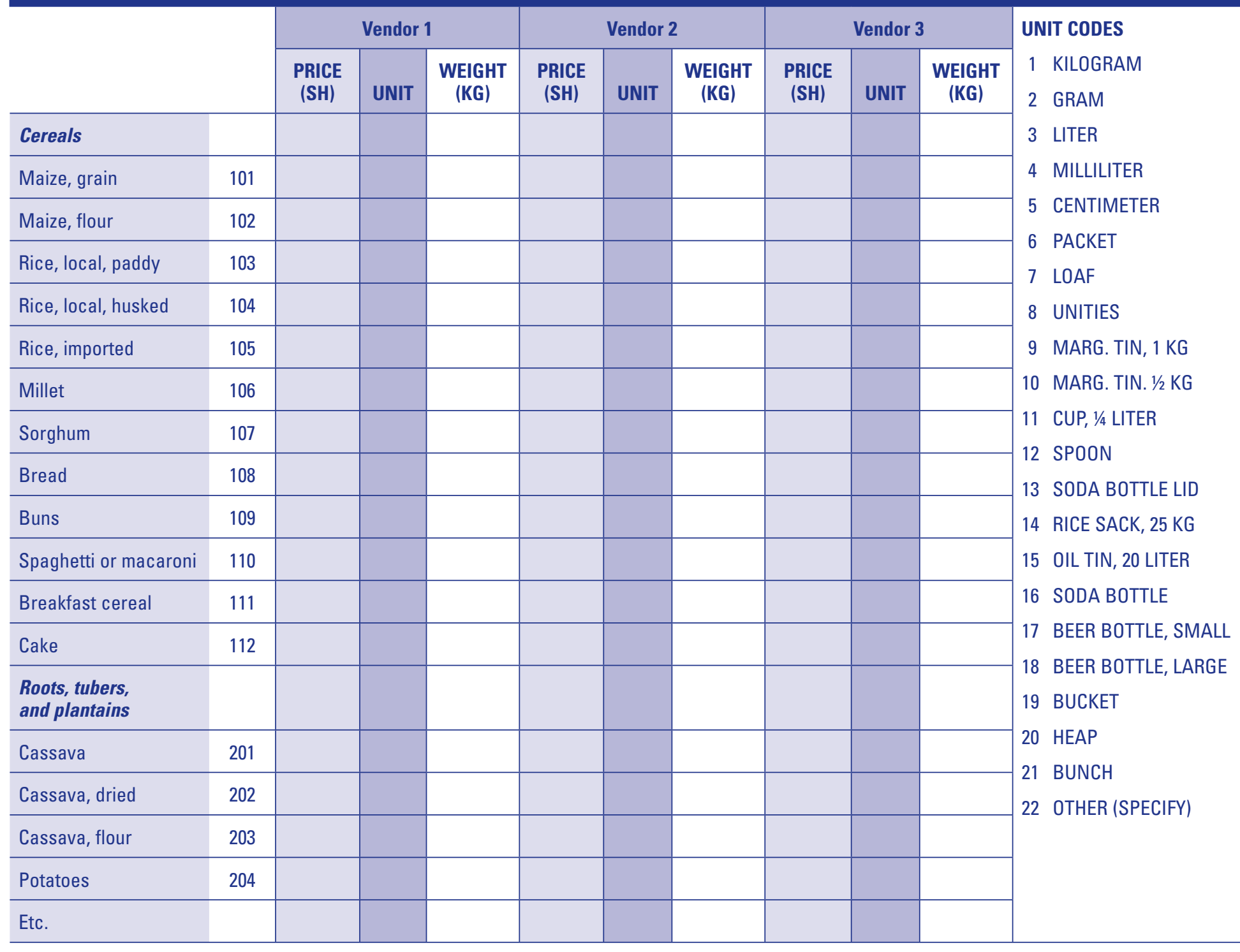

Note: Interviewer instructions are written in uppercase letters, while text to be read to respondents is written in lowercase letters. Etc. represents additional items that could be added to this type of survey.

52 Measuring Food Security Using Household Expenditure Surveys 


\section{Price Opinions}

In this method, knowledgeable community members or members of households participating in the expenditure survey themselves are the informants, whether from all households or only a subset within each community. The informants are asked to give their best estimate of the price per unit of the foods for which metric prices are needed. The method can be used with a focus group carefully chosen to represent average people in each community (not considerably richer or poorer). As always, when asking directly about prices, it is very important that the respondent understand that what is being asked for is the price per unit, with the unit of measure clearly indicated.

In a price opinion survey administered as part of the 1995-96 Papua New Guinea survey, household respondents were shown color photographs of 12 food items in the typical units of measure found in markets (for example, bundle, heap, or package) and asked to report their price (Gibson and Rozelle 2005). The question asked was "How much does it currently cost to buy a [name of item] like this in the main market or store in this village or town?" For foods for which scale was important, a box of matches was included in the photograph for perspective.

Gibson and Rozelle (2005) report that this method was much less expensive and less time-consuming than the market price survey conducted simultaneously and that the resulting prices were quite close to market prices. Note that for food items reported in local units of measure it is necessary to also collect information on the metric equivalent weight in order to compute metric quantities, which can be very time-consuming (see the first section of this chapter).

\section{Price Estimations from Unit Values}

In this method, a subset of survey households can be asked to report both their expenditures and the quantities of foods acquired, the latter of which must be converted to metric units if not already reported in them. This information can be used to calculate mean or median unit values (expenditures divided by quantities) for communities or higher-level geographical units that can then serve as prices.

When unit values are used to estimate prices, the prices may be biased for several reasons. First, they can contain considerable measurement error due to reporting, recording, and data entry errors in not just one piece of data, but the two pieces used to calculate them (expenditures and quantities). Because they are applied at the community or higher level, they also hide variation across households in prices paid due to differing qualities of foods acquired. ${ }^{17}$ Finally, because unit values are available only for the households that actually acquire a food, they are subject to sample selection bias. Households that did not acquire the food over the survey reference period may face systematically different prices than those that did (Deaton 1997, Capéau and Dercon 2005, Gibson and Rozelle 2005). These biases, in turn, can lead to inaccurate estimates of the quantity-based indicators of food security, such as food energy deficiency prevalences.

\footnotetext{
17 This particular issue leads to biased estimates of price elasticities derived from food demand equations. The picture-based price opinions methodology has the advantage over the unit
} value approach in that bias due to quality effects should be less because all informants are seeing the same picture (Gibson and Rozelle 2005). 
Employing survey data collected in rural Ethiopia in 1994-95, Capéau and Dercon (2005) find substantial differences between mean unit values and prices collected in a market survey for some foods and villages. Similarly, Gibson and Rozelle (2005) compare mean unit values with market prices using the 1995-96 Papua New Guinea survey and find substantial differences from market prices for some commonly consumed foods. They also report that price opinions are more congruent with market prices than are mean unit values.

Capéau and Dercon (2005) suggest a statistical method to estimate metric prices from unit values (see the fifth section of Chapter 5) that corrects for measurement error by making use of information on the distribution of unit values rather than just relying on means or medians. Applying this method to the rural Ethiopia survey data, they find that after this correction estimated prices are more congruent with market survey prices than are mean unit values.

These findings suggest that unit values should be avoided as the basis for price estimations in favor of market prices or price opinions. If it is necessary to use unit values, the Capéau and Dercon (2005) statistical technique should be applied if possible. There is reason to believe, however, that unit values carefully cleaned using the cleaning techniques laid out in this manual (see Chapter 5), while quite time-consuming to obtain, may not be such a poor substitute for market prices. When community mean unit values for eight foods from the 1995-96 Papua New Guinea survey data are compared to those reported by Gibson and Rozelle (2005), they are found to line up quite well with market survey prices. The divergence of sample means of unit values and market prices is less than 15 percent for all of the foods, with a mean (absolute value) difference of 7 percent (as compared to 13 percent reported by Gibson and Rozelle, with the highest being 29 percent). These results are reported in Appendix 5 of this manual.

\section{PRIMARY EQUIVALENTS OF PROCESSED FOODS}

For some foods, data are collected on the quantities acquired in both raw and processed forms. For example, households may be asked about the acquisitions of both maize grain and maize meal or flour. Yet in many cases analysts wish to estimate the total amount of a raw food acquired, which requires converting the amount of the processed foods to their "primary equivalent" value. For example, the grain-to-meal "extraction rate" for maize is 90 percent. Dividing the (metric) quantity of maize meal reported by 0.9 gives the maize grain equivalent.

Extraction rates can be found in FAO (no date). Country-specific "extraction rates" are first given, followed by commodity trees giving a worldwide range of extraction rates for commonly consumed foods. Note that because extraction rates can vary significantly by variety (for example, varieties of maize), data collected locally should be used whenever possible.

\section{ENERGY CONTENT OF FOODS AND EDIBLE PORTIONS}

Food composition tables are available for a large number of countries in the world. Because some foods have different calorie values depending on the geographic area (for example, meats, due to different fat contents) and a few foods are consumed only in specific 


\section{Box 4. Types of Foods Containing Inedible Parts}

Meat and poultry (inedible parts include bones, connective tissue, and gristle)

Fish (head, scales, skin, and guts)

Shellfish (shells)

Eggs (shells)

Nuts (shells)

Fruits and vegetables (peel, seeds, stems, cores, and outer leaves)

Roots and tubers (peel)

Grains (husk, if acquired unhusked)

Source: FSANZ 2004.

countries, country-specific tables should be used when possible.

However, because many tables contain erroneous values, care should be taken to have the values checked by a trained nutritionist before applying them. Another option is to compare them to another table and to check on any values that differ greatly.

Available food composition tables are listed in the INFOODS (International Network of Food Data Systems) "International Food
Composition Tables directory" (FA0 2006b). The WorldFood Dietary Assessment System contains energy values for a list of 1,800 foods for six countries (Egypt, India, Indonesia, Kenya, Mexico, and Senegal). It can be accessed online free of charge (FAO 2006c). Energy values from the USDA's food composition database, constructed using the most up-to-date techniques for determining energy composition, can also be found online (USDA 2005). The energy values used for FAO's food balance sheet calculations can also be found online (FA0 2006c).

The number of kilocalories of energy in the foods acquired by households is reported in food composition tables in units of 100 grams of the edible portion of the food. Thus, it is first necessary to obtain that portion before converting to its energy content. The edible portion is the part of the food that is available for consumption once inedible components have been removed. Many foods are acquired in their fully edible form, for example, maize grain and bread. However, many contain inedible parts that are removed prior to preparation. The types of foods that are generally not 100 percent edible are given in Box 4. Edible portions are reported in some food composition tables, but not all. ${ }^{18}$ Although they generally do not vary widely across populations, some may differ because different parts of a food are considered edible. Appendix 6 contains edible percentages from several developing countries and the United States for foods commonly consumed in developing countries.

${ }^{18}$ Some tables report refuse portions rather than edible portions 


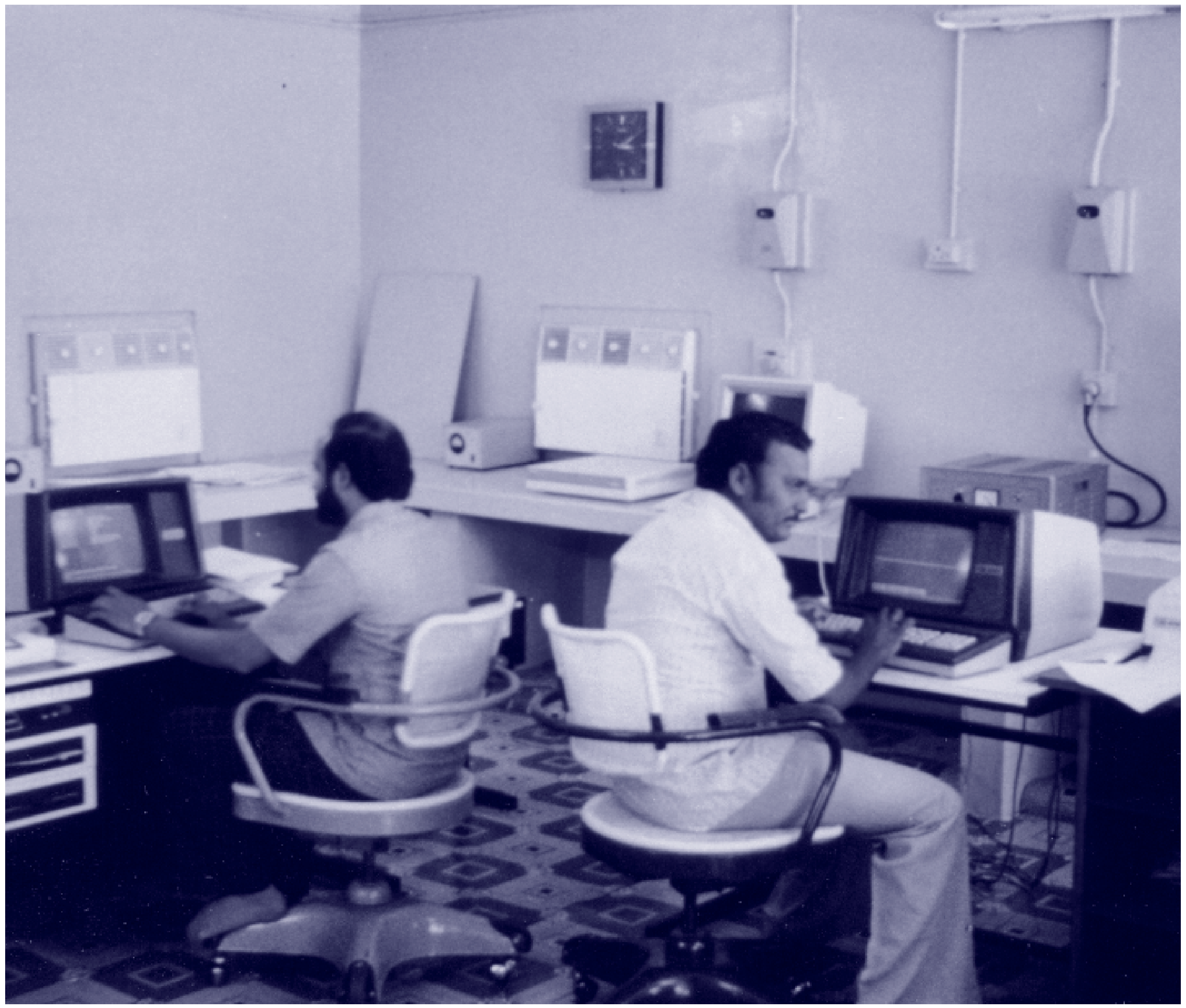




\section{Processing and Cleaning the Data}

A fter the data have been collected and entered into a computer, they must be processed and cleaned before food security indicators can be calculated. Box 5 summarizes the steps for doing so. Data cleaning is the process of detecting and removing errors incurred during the processes of reporting, recording, and entering data. Data users who are also part of the data collection process can replace any erroneous values that are detected with the correct value by referring back to questionnaires or even going back to households for clarification of responses. For data users who have only the raw data files at their disposal, options for dealing with erroneous values are discussed in this chapter.

\section{ASSEMBLING THE RAW DATA COLLECTED FROM HOUSEHOLDS AND INITIAL DATA CLEANING}

For the convenience of data entry, the raw data collected from households may be in various separate files arranged by variable (for example, the quantity and expenditure data may be in separate files), geographical location, food group, or food source (that is, purchased, home-produced, or acquired in kind). The first step in data processing is to assemble the data into one file. If the files were originally differentiated by food source, it is important to make sure that there is an identifying variable for the food source in the final file, because it may be needed for later data analysis.

\section{Box 5. Data Processing and Cleaning Steps}

1. Assemble the raw household data into one file, and conduct initial data cleaning.

2. Clean and process metric conversion factors, metric prices, and proportionate weights of ingredients in prepared dishes.

3. Calculate the number of household members and adult equivalents.

4. Calculate household energy requirements.

5. Calculate metric quantities of foods acquired by households. 6. Clean the metric food quantities.

7. Estimate metric food quantities to replace errors and missing values.

8. Calculate the total quantity of each food acquired daily by each household.

9. Estimate food-level expenditures to replace errors and missing values.

10. Calculate total daily household food expenditures.

11. Calculate total daily household energy acquisition.

12. Clean total daily household energy acquisition.

The recommended layout for the file is to give quantity and/or expenditure data by household, food item, and food source for each case. An example of a file layout in which quantity, unit of measure, 
and expenditure data are collected from households surveyed in a single-visit interview is given in Box 6.

The variables are defined as follows:

$$
\text { hhid }=\text { household identification number }
$$

foodcode $=$ food item code

source $=$ source of the food acquired (1: food purchased, 2: home-produced food consumed, 3: food received in kind)

qty = quantity of food acquired from the source over the reference period

unitcode $=$ unit of measure in which the quantity is reported

$\exp =$ expenditures on the food acquired from the source over the reference period

As part of initial data cleaning, the data for all variables should be examined for obvious erroneous or irrelevant values as follows:

1. Make sure that the "foodcode," "source," and "unitcode" data are all valid, that is, correspond to valid codes as printed on the questionnaire or added after data collection. Make sure that the unit codes are properly matched with food codes, checking for obvious errors (for example, bottles of bananas).

2. Make sure that the file contains only codes for food items. Any nonfood items should be dropped before proceeding further with data processing.

3. There should be no zero values for quantities or expenditures in the data file. Flag all cases for which either

- "qty" is zero and "exp" is nonzero or

- "exp" is zero and "qty" is nonzero.

\section{Box 6. Sample Layout of Data File with the Raw Data} Collected from Households

\section{\begin{tabular}{c|c|c|c|c|c|c} 
case & hhid & foodcode & source & qty & unitcode & $\exp$ \\
\hline
\end{tabular}}

\begin{tabular}{l|l|l|l|l|l|l}
1 & 101001 & 105 & 1 & 6 & 20 & 24000 \\
\hline 2 & 101001 & 107 & 1 & 3 & 20 & 12000 \\
\hline 3 & 101001 & 107 & 2 & 2 & 20 & 7500 \\
\hline
\end{tabular}

\begin{tabular}{r|r|r|r|r|r|l|}
\hline 3 & 101001 & 107 & 2 & 2 & 20 & 7500 \\
\hline 4 & 101001 & 125 & 1 & 15 & 3 & 9000 \\
\hline
\end{tabular}

\begin{tabular}{c|c|c|c|c|c|c}
4 & 101001 & 125 & 1 & 15 & 3 & 9000 \\
\hline 5 & 101001 & 139 & 1 & 2 & 1 & 2000 \\
\hline
\end{tabular}

\begin{tabular}{r|r|r|r|r|r|r}
\hline 6 & 101001 & 145 & 1 & 5 & 1 & 6500 \\
\hline 7 & 101001 & 147 & 1 & 4 & 54 & 400 \\
\hline
\end{tabular}

\begin{tabular}{r|r|r|r|r|r|l|}
\hline 7 & 101001 & 147 & 1 & 4 & 54 & 400 \\
\hline 8 & 101001 & 148 & 3 & 500 & 2 & 300 \\
\hline
\end{tabular}

\begin{tabular}{r|r|r|r|r|r|l}
\hline 9 & 101002 & 101 & 1 & 1 & 68 & 1000 \\
\hline
\end{tabular}

\begin{tabular}{l|l|l|l|l|l|l}
\hline 10 & 101002 & 105 & 1 & 1 & 20 & 3500 \\
\hline
\end{tabular}

\begin{tabular}{l|l|l|l|l|l|l}
\hline 11 & 101002 & 110 & 1 & 1 & 1 & 1000 \\
\hline 12 & 101002 & 125 & 1 & 8 & 3 & 4000 \\
\hline
\end{tabular}

\begin{tabular}{r|r|r|r|r|r|l}
12 & 101002 & 125 & 1 & 8 & 3 & 4000 \\
\hline 14 & 101002 & 127 & 1 & 3 & 4 & 300 \\
\hline
\end{tabular}

\begin{tabular}{r|r|r|r|r|r|l}
\hline 14 & 101002 & 133 & 1 & 1 & 66 & 100 \\
\hline 15 & 101002 & 134 & 1 & 3 & 64 & 300 \\
\hline 16 & 101002 & 139 & 1 & 2 & 1 & 1400 \\
\hline 17 & 101002 & 151 & 1 & 2 & 33 & 1000 \\
\hline 18 & 101003 & 109 & 1 & 1 & 64 & 1000 \\
\hline 19 & 101003 & 110 & 1 & 1 & 1 & 1000 \\
\hline 20 & 101003 & 114 & 2 & 1 & 42 & 900 \\
\hline 21 & 101003 & 117 & 1 & 0.5 & 1 & 1000 \\
\hline 22 & 101003 & 123 & 1 & 1 & 55 & 600 \\
\hline 23 & 101003 & 124 & 1 & 8 & 85 & 800 \\
\hline 24 & 101003 & 141 & 1 & 0.5 & 1 & 700 \\
\hline 25 & 101003 & 148 & 2 & 0.25 & 51 & 63 \\
\hline
\end{tabular}

Note: See text for definition of variables. 
Examine the data, and determine whether it is best to set both "qty" and "exp" to "missing" for later replacement for these cases or only the zero value to "missing" (if it is determined that the corresponding nonzero values are valid). ${ }^{19}$

In some situations there may be a very large number of cases with zero values for both quantities and expenditures or missing values for both quantities and expenditures. The data were probably entered in such a way that there are cases for each food for each household, even those households that did not acquire the food. If this is so, drop the cases. ${ }^{20}$

4. Make sure there are no negative values for "qty" or "exp."

5. If the monetary value method is being used for a food or a food is a prepared dish consumed away from home, check to make sure that "exp" is positive and "qty" is missing for all cases for the food.

For data users not involved in data collection, it is important to determine whether the quantity and expenditure data may have been processed before the dataset was released. In some cases the raw quantity and expenditures data have been transformed to daily or monthly values. If so, this should be taken into account in the subsequent data processing or the data should be transformed back to their raw form before proceeding.

\section{PROCESSING AND CLEANING METRIC CONVERSION FACTORS, METRIC PRICES, AND PROPORTIONATE WEIGHTS OF INGREDIENTS IN PREPARED DISHES}

As explained in Chapter 3, metric conversion factors are needed for calculating metric food quantities when the "local units of measure (variable size)" and "count" methods of data collection are used. Metric prices are needed for calculating metric quantities when the "monetary value" method of data collection is used. Metric prices of prepared dishes and proportionate weights of their ingredients are needed for calculating the metric weights of foods that are contained in prepared dishes. This section shows how to clean and process these data when they have been collected in markets located close to the communities (also known as villages or neighborhoods) of surveyed households. These communities are referred to as "primary sampling units" or PSUs, a term used to denote the units that are first randomly chosen before households are selected as part of the commonly applied two-stage stratified random sampling strategy. This method can be applied to data that have been collected at the level of higher geographical areas as well (for example, administrative districts or zones).

${ }^{19}$ In some cases an attempt is made to collect data on food consumption rather than food acquisition. This often leads to erroneous reporting of zero values by households (see the second section of Chapter 3).

${ }^{20}$ Note that general percentages of households acquiring foods can still be calculated later by restructuring the data file such that the variables are foods and the cases are households, with one case per household (this can be achieved using the "reshape" command in STATA). After doing so, the households not acquiring a food will have missing values for both quantity and expenditure that can be replaced by zero values. Thus, there is no need to keep households with zero values for both quantities and expenditures in the data file. 


\section{Metric Conversion Factors}

Box 7 gives an example of a data file containing metric conversion factors, or weights, collected in PSUs within broader geographical areas called districts. As specified in the first section of Chapter 4, it is best to collect multiple weights for the same food and unit of measure in each market.

The variables are defined as follows:

$$
\begin{aligned}
\text { foodcode }= & \text { food item code } \\
\text { unitcode }= & \text { unit of measure in which metric weight is } \\
& \text { recorded } \\
\text { district }= & \text { district in which PSU is located } \\
\text { PSU }= & \text { primary sampling unit (or community) in } \\
& \text { which data were collected } \\
\text { sample }= & \begin{array}{l}
\text { number identifying the sample taken (needed } \\
\text { with multiple samples) }
\end{array} \\
\text { weight }(\mathrm{kg})= & \text { weight in kilograms of the food in the unit of } \\
& \text { measure }
\end{aligned}
$$

The steps for data cleaning and processing are as follows:

1. Check to make sure all food and unit codes are valid and not missing. If not, correct or drop the case.

2. Drop any cases for which the weight variable is missing or equal to zero

3. Tabulate the kilogram weights of each food in each unit of measure to detect outliers. If there is a lot of variability in the data, this may be best done by geographical region (for example, districts in the example data file)

4. Correct outlying values, or drop the cases with outliers entirely.

\section{Box 7. Sample Layout of Data File with Metric Conversion Factors Collected in Markets}

\begin{tabular}{l|l|l|l|l|l|l} 
case & foodcode & unitcode & district & PSU & sample & weight $(\mathrm{kg})$ \\
\hline
\end{tabular}

\begin{tabular}{c|c|c|c|c|c|c}
\hline 1 & 105 & 3 & 1 & 1 & 1 & 2.46 \\
\hline 2 & 105 & 3 & 1 & 1 & 2 & 2.21 \\
\hline 3 & 105 & 3 & 1 & 1 & 3 & 2.64 \\
\hline 4 & 105 & 3 & 1 & 2 & 1 & 3.01 \\
\hline 5 & 105 & 3 & 1 & 2 & 2 & 2.56 \\
\hline 6 & 105 & 3 & 1 & 2 & 3 & 2.45 \\
\hline 7 & 105 & 3 & 1 & 3 & 1 & 3.26 \\
\hline 8 & 105 & 3 & 1 & 3 & 2 & 3.52 \\
\hline 9 & 105 & 3 & 1 & 3 & 3 & 2.98 \\
\hline 10 & 105 & 3 & 2 & 1 & 1 & 3.24 \\
\hline 11 & 105 & 3 & 2 & 1 & 2 & 3.36 \\
\hline 12 & 105 & 3 & 2 & 1 & 3 & 2.99 \\
\hline 13 & 105 & 3 & 2 & 2 & 1 & 2.97 \\
\hline 14 & 105 & 3 & 2 & 2 & 2 & 82.6 \\
\hline 15 & 105 & 3 & 2 & 2 & 3 & 3.45 \\
\hline 16 & 105 & 3 & 3 & 1 & 1 & 3.02 \\
\hline 17 & 105 & 3 & 3 & 1 & 2 & 2.65 \\
\hline 18 & 105 & 3 & 3 & 1 & 3 & 2.46 \\
\hline 19 & 105 & 5 & 3 & 2 & 1 & 10.02 \\
\hline 20 & 105 & 5 & 3 & 2 & 2 & 11.85 \\
\hline 21 & 105 & 5 & 3 & 2 & 3 & 9.06 \\
\hline
\end{tabular}

Note: See text for definition of variables.

5. Calculate the mean (or median) conversion factor for each food, unit of measure, and market.

6. If there are any remaining missing values for conversion factors for a community, estimate them using the mean (or median) factor for the rest of the cases in a geographical area (for example, a district).

60 Measuring Food Security Using Household Expenditure Surveys 
Where the local units of measure (fixed size), volumetric equivalents, linear dimensions, and food models methods of data collection are used, there will be only one metric conversion factor for each food, because these factors will not vary across geographical areas. These data can be examined for obvious errors.

\section{Metric Prices}

Box 8 gives an example of a data file containing prices collected at the community level within broader districts. As specified in the fourth section of Chapter 4 , it is best to collect the price data for the same food and unit of measure from multiple vendors in each market. Data are collected from each vendor on the weight of the food in the unit of measure and the price per unit.

The variables are defined as follows:

foodcode $=$ food item code

unitcode $=$ unit of measure in which price is recorded

district $=$ district in which PSU is located

PSU = primary sampling unit (or community) in which data were collected

vendor $=$ vendor from whom food was acquired

weight $(\mathrm{kg})=$ weight in kilograms of the food in the unit of measure

price $=$ price of the food in the unit of measure

The steps for data cleaning and processing are as follows:

1. Check to make sure all food codes and unit codes are valid. If not, correct or drop the case.

2. Drop any cases for which the weight or price variable is missing or equal to zero.

\section{Box 8. Sample Layout of Data File with Price Data} Collected in Markets

\begin{tabular}{|c|c|c|c|c|c|c|c|}
\hline case & foodcode & unitcode & district & PSU & vendor & $\begin{array}{c}\text { weight } \\
(\mathrm{kg})\end{array}$ & price \\
\hline 1 & 105 & 3 & 1 & 1 & 1 & 2.46 & 60 \\
\hline 2 & 105 & 3 & 1 & 1 & 2 & 2.21 & 55 \\
\hline 3 & 105 & 3 & 1 & 1 & 3 & 2.64 & 55 \\
\hline 4 & 105 & 3 & 1 & 2 & 1 & 3.01 & 60 \\
\hline 5 & 105 & 3 & 1 & 2 & 2 & 2.56 & 60 \\
\hline 6 & 105 & 3 & 1 & 2 & 3 & & \\
\hline 7 & 105 & 3 & 1 & 3 & 1 & 3.26 & 50 \\
\hline 8 & 105 & 3 & 1 & 3 & 2 & 3.52 & 55 \\
\hline 9 & 105 & 3 & 1 & 3 & 3 & 2.98 & 50 \\
\hline 10 & 105 & 3 & 2 & 1 & 1 & 3.24 & 45 \\
\hline 11 & 105 & 3 & 2 & 1 & 2 & 3.36 & 45 \\
\hline 12 & 105 & 3 & 2 & 1 & 3 & 2.99 & 45 \\
\hline 13 & 105 & 3 & 2 & 2 & 1 & 2.97 & 40 \\
\hline 14 & 105 & 3 & 2 & 2 & 2 & 82.6 & 40 \\
\hline 15 & 105 & 3 & 2 & 2 & 3 & 3.45 & 45 \\
\hline 16 & 105 & 3 & 3 & 1 & 1 & 3.02 & 60 \\
\hline 17 & 105 & 3 & 3 & 1 & 2 & 2.65 & 60 \\
\hline 18 & 105 & 3 & 3 & 1 & 3 & 2.46 & 60 \\
\hline 19 & 105 & 5 & 3 & 2 & 1 & 10.02 & 240 \\
\hline 20 & 105 & 5 & 3 & 2 & 2 & 11.85 & 220 \\
\hline 21 & 105 & 5 & 3 & 2 & 3 & 9.06 & 240 \\
\hline 22 & 105 & 5 & 3 & 3 & 1 & 8.23 & 200 \\
\hline 23 & 105 & 5 & 3 & 3 & 2 & 9.68 & 200 \\
\hline 24 & 105 & 5 & 3 & 3 & 3 & & \\
\hline 25 & 105 & 5 & 3 & 4 & 1 & 11.05 & 275 \\
\hline
\end{tabular}

Note: See text for definition of variables. 
3. Calculate the price per kilogram for all cases. Note: For data cleaning purposes it may be preferable to use kilogram (as opposed to gram) prices, because error detection with them is less tiresome to the eyes.

4. Tabulate the price per kilogram of each food to detect outliers. Correct or drop the cases with outliers. If there is a lot of variability in the data, this may be best done for subregions within the population being sampled. Also note that urban and rural prices can differ greatly.

5. Calculate the mean (or median) metric price for each food and community.

6. If there are any remaining missing values for a community, estimate them using the mean (or median) price for the rest of the cases in a geographical area (for example, a district). ${ }^{21}$

\section{Metric Prices of Prepared Dishes and Proportionate Weights of Their Ingredients}

Box 9 gives an example of a data file containing the metric prices of prepared dishes and proportionate weights of their ingredients. A weight must be available for each food listed as an ingredient in each dish reported by households as consumed outside the home. In the example, proportionate weights have been calculated for multiple vendors within communities located close to sample households.

The variables are defined as follows:

$$
\begin{aligned}
\text { foodcode }= & \text { food item code } \\
\text { district }= & \text { district in which PSU is located } \\
\text { PSU }= & \text { primary sampling unit (or community) in } \\
& \text { which data were collected } \\
\text { vendor }= & \text { vendor from whom information was collected } \\
\text { price }(\mathrm{g})= & \text { price per gram of the dish } \\
\text { pw106 = } & \text { proportionate weight of food } 106 \text { in the dish's } \\
& \text { total weight } \\
\text { pw107 }= & \text { proportionate weight of food } 107 \text { in the dish's } \\
& \text { total weight } \\
\text { pw303 }= & \text { proportionate weight of food } 303 \text { in the dish's } \\
& \text { total weight } \\
\text { pw304 }= & \text { proportionate weight of food } 304 \text { in the dish's } \\
& \text { total weight }
\end{aligned}
$$

The steps for data cleaning and processing are as follows:

1. Check to make sure all food codes are valid. If not, correct or drop the case.

2. Drop any cases for which the price or proportionate weight variable is missing or equal to zero.

3. Tabulate the price per gram and proportionate weights of each dish to detect outliers. Correct or drop the cases with outliers. If there is a lot of variability in the data, this may be best done for subregions within the population being sampled. Also note that urban and rural prices can differ greatly.

${ }^{21}$ In this calculation, communities with more vendors may be overrepresented (there are less likely to be missing values), which may bias the price where the density of vendors is related to price levels. If this is the case, it is important to use weighted mean prices, taking into account the sampling design of the survey, to make sure the prices are representative of the entire geographical area. 
Box 9. Sample Layout of Data File with Metric Prices of Prepared Dishes and Proportionate Weights of Their Ingredients

\begin{tabular}{|c|c|c|c|c|c|c|c|c|c|}
\hline case & foodcode & district & PSU & vendor & price $(g)$ & pw106 & pw107 & pw303 & pw304 \\
\hline 1 & 1301 & 1 & 1 & 1 & 4000 & 0.19 & 0 & 0 & 0 \\
\hline 2 & 1301 & 1 & 1 & 2 & 3450 & 0.3 & 0 & 0 & 0 \\
\hline 3 & 1301 & 1 & 1 & 3 & 3800 & 0.25 & 0 & 0 & 0 \\
\hline 4 & 1301 & 1 & 2 & 1 & 4500 & 0.31 & 0 & 0 & 0 \\
\hline 5 & 1301 & 1 & 2 & 2 & 4500 & 0.28 & 0 & 0 & 0 \\
\hline 6 & 1301 & 1 & 2 & 3 & 3700 & 0.15 & 0 & 0 & 0 \\
\hline 7 & 1301 & 1 & 3 & 1 & 4000 & 0.25 & 0 & 0 & 0 \\
\hline 8 & 1301 & 1 & 3 & 2 & 4500 & 0.28 & 0 & 0 & 0 \\
\hline 9 & 1301 & 1 & 3 & 3 & 3800 & 0.35 & 0 & 0 & 0 \\
\hline 10 & 1302 & 1 & 1 & 1 & 2500 & 0.3 & 0.6 & 0 & 0.1 \\
\hline 11 & 1302 & 1 & 1 & 2 & 2400 & 0.33 & 0.52 & 0 & 0.15 \\
\hline 12 & 1302 & 1 & 1 & 3 & 2200 & 0.25 & 0.63 & 0 & 0.12 \\
\hline 13 & 1302 & 1 & 2 & 1 & 3000 & 0.26 & 0.65 & 0 & 0.09 \\
\hline 14 & 1302 & 1 & 2 & 2 & 3100 & 0.29 & 0.62 & 0 & 0.09 \\
\hline 15 & 1302 & 1 & 2 & 3 & 2200 & 0.34 & 0.58 & 0 & 0.08 \\
\hline 16 & 1302 & 1 & 3 & 1 & 2300 & 0.33 & 0.57 & 0 & 0.1 \\
\hline 17 & 1302 & 1 & 3 & 2 & 2300 & 0.32 & 0.55 & 0 & 0.13 \\
\hline 18 & 1302 & 1 & 3 & 3 & 3000 & 0.3 & 0.56 & 0 & 0.14 \\
\hline 19 & 1303 & 1 & 1 & 1 & 500 & 0 & 0 & 0.75 & 0.25 \\
\hline 20 & 1303 & 1 & 1 & 2 & 550 & 0 & 0 & 0.78 & 0.22 \\
\hline 21 & 1303 & 1 & 1 & 3 & 450 & 0 & 0 & 0.67 & 0.33 \\
\hline 22 & 1303 & 1 & 2 & 1 & 425 & 0 & 0 & 0.76 & 0.24 \\
\hline 23 & 1303 & 1 & 2 & 2 & 400 & 0 & 0 & 0.78 & 0.22 \\
\hline 24 & 1303 & 1 & 2 & 3 & 550 & 0 & 0 & 0.80 & 0.2 \\
\hline 25 & 1303 & 1 & 3 & 1 & 400 & 0 & 0 & 0.65 & 0.35 \\
\hline
\end{tabular}

Note: See text for definition of variables. 
4. Calculate the mean (or median) metric price and proportionate weights for each dish and community.

5. If there are any remaining missing values for a community, estimate them using the mean (or median) price for the rest of the cases in a geographical area (for example, a district).

\section{CALCULATING THE NUMBER OF HOUSEHOLD MEMBERS, ADULT EQUIVALENTS, AND ENERGY REQUIREMENTS}

Data on the number of household members, adult equivalents, and energy requirements are all needed to enable data cleaning or indicator calculation, as explained in this section. Appendix 7 contains a STATA program for calculating the number of adult equivalents and household energy requirements.

\section{Number of Household Members and Adult Equivalents}

The number of household members or household size is needed for calculating per capita measures of household energy availability and consumption of individual foods. Dividing by household size adjusts for the number of people for which the energy or food is available. Calculating household size is straightforward: the number of members of each household is counted, with that number conforming to the definition of "household member" for the survey.

The number of "adult equivalents" is also used to adjust for the people for whom energy or food is available, but the fact that individual food needs vary by age and sex is taken into account. This number is used to adjust the various measures calculated at the household level (metric food quantities and energy acquisition) and thus assist in data cleaning by allowing comparisons across households that control for age and sex differences between them. Each person in the household is assigned an adult equivalent factor that compares his or her energy needs with those of a 30- to 60-year-old male according to the energy requirements for moderate activity given in Table 7.22 Using data collected as part of the household roster section of an HES, the number of adult equivalents is calculated as follows:

1. Assign an age-sex category to each individual in the sample; the categories are as defined in Table 7. Note that the energy requirements of children under age five do not depend on their sex. If age and sex data are missing, it may be necessary to impute them.

2. Calculate the number of household members in each age-sex category in each household.

3. Multiply the number of household members in each category by the corresponding adult equivalent factor, which is the energy requirement divided by the energy requirement for adult males 30-60 years old (2,900 kilocalories).

4. Sum the number of adult equivalents to obtain the total number of adult equivalents for each household.

\section{Household Energy Requirements}

As discussed in Chapter 2, use of the energy requirements for light activity is recommended. Table 7 reports these requirements by

${ }^{22}$ The requirements for light or heavy activity could be used if appropriate to the population. 
Table 7. Recommended daily caloric intake, by age group

Age group (years)

Kilocalories per day

Young children

\begin{tabular}{lc}
$<1$ & 820 \\
\hline $1-2$ & 1,150 \\
\hline $2-3$ & 1,350 \\
\hline $3-5$ & 1,550
\end{tabular}

\begin{tabular}{ll|l} 
Age group (years) & \multicolumn{2}{c}{ Kilocalories per day } \\
Older children & Boys & Girls \\
$5-7$ & 1,850 & 1,750 \\
\hline $7-10$ & 2,100 & 1,800 \\
\hline $10-12$ & 2,200 & 1,950 \\
\hline $12-14$ & 2,400 & 2,100 \\
\hline $14-16$ & 2,650 & 2,150 \\
\hline $16-18$ & 2,850 & 2,150
\end{tabular}

Age group (years)

Kilocalories per day

\begin{tabular}{lc|c|c} 
Adults: men & Light activity & Moderate activity & Heavy activity \\
$18-30$ & 2,600 & 3,000 & 3,550 \\
\hline $30-60$ & 2,500 & 2,900 & 3,400 \\
\hline$>60$ & 2,100 & 2,450 & 2,850 \\
Adults: women & Light activity & Moderate activity & Heavy activity \\
$18-30$ & 2,000 & 2,100 & 2,350 \\
\hline $30-60$ & 2,050 & 2,150 & 2,400 \\
\hline$>60$ & 1,850 & 1,950 & 2,150
\end{tabular}

Source: FAO, WHO, and UNU (1985), as published in Hoddinott (2001). 
age and sex, along with the requirements for moderate and heavy activity, as recommended by the 1981 expert consultation on energy and protein requirements (FAO, WHO, and UNU 1985). Note that the 2001 expert consultation on human energy requirements (UNU, WHO, and FAO 2004) provided updated energy requirements that can be found in Appendix 8.

Using the same age and sex categories used for calculating the number of household adult equivalents, each household's total energy requirement is calculated by multiplying the number of household members in each age-sex category by the corresponding energy requirement and summing these across household members. An additional 500 calories is added for each child younger than one year old to account for the greater energy needs of breastfeeding mothers.

\section{CALCULATING AND Cleaning DAILY HOUSEHOLD METRIC FOOD QUANTITIES}

\section{Calculating Metric Quantities from the Raw Food Data}

The next data processing step is to convert the food quantity or expenditures data collected by households to metric food quantities. In doing so, one metric weight unit of measure must be used consistently as the target for all conversions. The goal is for all food quantities to be in this unit of measure when the conversion process is complete. In the following sections, the target unit of measure is grams; the reported quantity is referred to as "qty," the reported expenditure as "exp," and the new quantity in grams as "qty_g."

The conversion calculation and source of data for undertaking the conversions depend, of course, on the method of data collection, as follows.
Metric units. If the quantities reported by households are in kilograms, then they are multiplied by 1,000 to give gram metric quantities:

$$
\text { qty_g = qty } \times 1000 \text {. }
$$

If the quantities reported by households are in milliliters, they are multiplied by a milliliters-to-grams conversion factor (MGCF) to give gram metric quantities:

$$
\text { qty_g = qty } \times \text { MGCF. }
$$

If the quantities reported by households are in liters, they are multiplied by the MGCF and 1,000 to give gram metric quantities:

$$
\text { qty_g = qty } \times \text { MGCF } \times 1000 \text {. }
$$

The conversion factors can be entered into a data file that is merged with the household data file, matching cases by food and unitcode, or, if there are few food items, they can be written into the program code being used for converting to gram metric quantities. For an example in STATA 8.0, the code for converting quantities of fruit juice (foodcode 2005) reported in milliliters is

$$
\text { generate qty_g }=\text { qty } * 1.05 * 1000 \text { if foodcode }==2005 \text {. }
$$

The conversion factor is taken from Appendix 4.

Monetary value. The expenditures reported by households must be matched with the metric prices by food and target geographical area. The expenditures are then divided by the metric prices to give metric quantities:

$$
\text { qty_g }=\text { exp/price_g. }
$$

In some surveys only expenditure data are collected for the food category "other" associated with each food group. In this case, the 
monetary value method must be used, and prices must be imputed using the mean (or median) value of prices for the prelisted foods in the food category. If household-level unit values can be calculated for these other foods, they will serve as the prices (see the following section on cleaning the metric food quantities).

Local units of measure (variable size) and counts. The quantities reported by households must be matched with metric conversion factors by food, unit of measure, and target geographical area. The reported quantities are then multiplied by the conversion factors to give metric quantities:

$$
\text { qty_g }=\text { qty } \times \text { MCF. }
$$

Local units of measure (fixed size), volumetric equivalents, linear dimensions, and food models. The quantities reported by households must be matched with metric conversion factors by food and unit of measure only. The reported quantities are then multiplied by the conversion factors to give metric quantities:

$$
\text { qty_g }=\text { qty } \times \text { MCF. }
$$

Foods consumed outside the bome. The expenditures on prepared dishes reported by households must be matched with their metric prices and the proportionate weights of their ingredients by food and target geographical area. Then follow these steps:

1. Place the food codes corresponding to foods consumed outside the home in a separate data file.

2. Calculate the metric weight of each dish as

$$
\text { qty_g_dish }=\text { exp/price_g. }
$$

3. Calculate the metric weight of each ingredient in the dish as

$$
\begin{aligned}
& \text { qty_g_foodcodel }=\text { qty_g_dish } \times \text { pwfoodcodel }, \\
& \text { qty_g_foodcode } 2=\text { qty_g_dish } \times \text { pwfoodcode } 2, \ldots \\
& \text { qty_g_foodcodeN }=\text { qty_g_dish } \times \text { pwfoodcodeN },
\end{aligned}
$$

where pwfoodcodel-pwfoodcodeN are the proportionate weights of foods in each dish.

4. Restructure the data file so that the cases are quantities of foods acquired by each household, each identified by its foodcode. The "reshape" command in STATA 8.0 can be used [reshape long qty_g, i(hhid) j(foodcode)]. The new file will have variables hhid foodcode qty_g.

5. Delete all cases with missing values.

6. Append the file to the main data file (using the "append" command in STATA). Note that if data were collected only on the total amount spent on food consumed outside of the home, it is not possible to compute metric food quantities for this food source.

\section{Cleaning the Metric Food Quantities}

Unit value cleaning. All of the methods of data collection except the "monetary value" method involve collection of data on food quantities acquired and associated expenditures. In this case, systematic cleaning of the quantity and expenditures data is possible because the metric unit values - that is, expenditures divided by metric quantity — of foods acquired by households located close to one another should be somewhat similar. This is because unit values are effectively the price that the household paid or would have 
to pay (in the case of home-produced or in-kind foods) and, even allowing for differences due to quality, negotiating skills, personal relationships, and so forth, prices for the same food should not vary greatly across households located close to one another. If a unit value seems to fall far from others, that could be due to error in the quantity or expenditure or both.

Metric (gram) unit values are calculated as

$$
\text { uv_g }=\text { exp/qty_g. }
$$

After computation of the unit values, the data cleaning steps are as follows:

1. (Optional) If there is a very large sample, time can be saved by first screening for major outliers by food, for example, those lying more than five standard deviations above the median, before the detailed examination of the data.

2. Where variability in unit values for a food is very great, this may reflect strong differences across geographical areas, in which case the cleaning should be conducted separately for different geographical areas, including urban and rural areas where relevant. To determine where this is necessary, run a histogram on the unit values for each food. There may be some foods for which there is very little variation in prices and examination by geographical area will waste time. For example, in some areas sugar is sold at a single price, so the unit values for this food should be very close to one another for the entire sample.

3. Examine the unit values by food (and geographical region where needed), looking for outliers at both ends of the distribution, that is, those that lie far from the lowest values and the highest values. (Note: this is very time-consuming, depend- ing on the number of foods.) In STATA, the "histogram" and "tabulate" commands can be used to detect outliers. Flag the outliers.

4. For the flagged cases, set the corresponding metric quantities and expenditures to missing values.

Note: If the original metric quantities are in grams, it may be desirable to temporarily convert them to kilograms for the unit value cleaning, because outlying values can be easier to detect visually for some currencies.

Cleaning the per adult equivalent quantities. Further data cleaning can be undertaken using metric food quantities per adult equivalent, calculated as quantity divided by the number of adult equivalents. Before this is done, the reported quantities acquired of each food for each household should be summed. This can be done using the "collapse" command in STATA. The reason a household may have multiple cases for the same food item is that it was acquired from more than one source (purchases, home production, or in-kind payment). Further, it may have been acquired for both in-home and outside-the-home consumption.

A criterion for outliers should be set and all values deemed outliers corrected or set to missing for later estimation. Note that only the values lying far above the others can be detected as possible errors here, because values falling far below a group are inherently valid (households can legitimately acquire small amounts of a food). These cases might be errors because households have reported harvests of home-produced foods over the reference period rather than their consumption of them and because data entry operators may have added extra zeros onto a number. In the AFINS project data cleaning, all values greater than three standard deviations from 
the median for each food were deemed outliers and set to missing for later estimation.

\section{Estimating Metric Quantities to Replace Missing Values}

Following the data cleaning process outlined earlier, there will be missing values remaining among the computed metric quantities of food acquired by households. If these are not replaced by estimated values, it will not be possible to compute most of the food security indicators for the household.

There are two basic approaches to imputing values for missing cases. The first is to replace the values with the mean or median value for the other households in the sample or for other households in the same geographical location. The second is to replace the values with the mean or median value of the other households having the same demographic characteristics, including location. The latter is more likely to give values close to the true quantity acquired by the household and helps to preserve the true variation in the data.

The second approach, implemented as part of the AFINS project data processing, uses food-specific ordinary least squares (OLS) regression equations in which the independent variables are household characteristics, such as age and sex structure; education; and economic status in addition to location. Because characteristics specific to each household are taken into account, this method gives more accurate estimates. Further, it helps to preserve the true variation in the data. Predicted values generated by the regression serve as the estimates to replace missing values. Appendix 9 contains an example of STATA code for implementing this method.

\section{Calculating Total Daily Quantities of Foods Acquired}

If it was not already done before cleaning quantities per adult equivalent, the reported quantities for each household and food code should be summed using the "collapse" command in STATA. To calculate daily quantities of food acquired, the cleaned and summed metric quantities for each household and food item are divided by the number of days in the reference period for food data collection.

\section{CALCULATING DAILY HOUSEHOLd TOTAL FOOD EXPENDITURES}

\section{Estimating Expenditures to Replace Missing Values}

After unit values have been cleaned, they can be used to estimate prices for replacing errors and missing values in the expenditures data. The simplest way to do this is to calculate the mean or median of the unit values for each food acquired in a geographical area, starting with the lowest area possible, which is often a community or a primary sampling unit of a survey. It is best to establish a cutoff for the minimum number of cases that will be used to compute a unit value. For AFINS project data processing, the minimum number used was five. In this case, if there are not five available for a community, a median is calculated for the next geographical level up, for example, the district, and so on.

Alternatively, when the statistical expertise is available, an econometric method developed by Capéau and Dercon (2005) for simultaneously estimating local unit conversion factors and 
prices reported in local units can be applied. ${ }^{23}$ Metric prices can be estimated for all foods and communities for which they are missing if sufficient nonmissing data are available from households in the same community and at least some households report their acquisitions of the food in a metric unit. This method has the added advantage that it corrects for underlying measurement error in the expenditures and quantity data employed (see the fourth section of Chapter 4). The working paper version of Capéau and Dercon (2005), which can be found free online, is Capéau and Dercon (2004).

Expenditures are reported directly by households, so the only processing that needs to be done is to impute expenditures to replace values set to missing during the unit value cleaning described above or that were originally missing. They are estimated by matching the unit values computed for foods and geographical areas with those in the main dataset containing the household data. For cases with missing values, the cleaned quantity data are then multiplied by the estimated metric prices to arrive at the estimated expenditure. It is important, of course, to make sure that the metric unit of measure used for the food quantities and prices is the same (for example, both in grams or kilograms). For surveys in which only expenditure data are available, the source of prices is market survey prices, prices collected using price opinions, or prices from a secondary source, such as consumer price index prices.

\section{Calculating Total Daily Food Expenditures}

To calculate total food expenditures, the reported expenditures for each household must first be summed (using the "collapse" command in STATA). Daily expenditures are then calculated by dividing by the number of days in the reference period for food data collection.

\section{CALCULATING AND CLEANING DAILY HOUSEHOLD ENERGY ACQUISITION}

Calculation of the total energy content of the food acquired by households starts by merging the data file containing the cleaned daily metric food quantities with that containing the energy conversion factors and edible portions, matching cases by food code. The metric quantities are then multiplied by the edible portion ( $\mathrm{a}$ ratio between 0 and 1) of each food and the energy conversion factors. Given that the energy conversion factors (ECF) are per 100 grams of edible portion, the formula for the calculation is

$$
\text { kcals }=\text { qty_g_d } \times \text { edibleportion } \times(\mathrm{ECF} / 100),
$$

where qty_g_d are the cleaned daily metric food quantities. Next, the energy values are added up across all foods acquired by each household to arrive at total daily household energy acquisition.

There may be some households with missing energy acquisition data due to missing metric food quantity data for at least one food. This can happen, for example, if a missing metric quantity cannot be computed using the regression technique for estimating values based on household characteristics because there are too few cases of a food for running a regression or a household has missing values for many of the household characteristics. For these households energy acquisition can be estimated employing the same OLS regression

${ }^{23}$ Local unit conversion factors and prices are estimated using an econometric specification with a multiplicative disturbance term that is log-normally distributed, which corrects for heteroskedasticity. Further, a correction proposed by Goldberger (1968) that is necessary for calculating unbiased estimates of conversion and prices from the estimated coefficients is applied. 
technique used for imputing missing metric quantities (see the previous section), with the dependent variable calorie acquisition per adult equivalent.

At this point processors may also wish to drop some households deemed to have unreliable data. In the AFINS project data processing, all households with missing values for more than 50 percent of the foods they acquired, as detected in the metric quantities data processing, were dropped. Additionally, all households with computed energy acquisition per adult equivalent of greater than 12,000 kilocalories were dropped. This could happen, for example, if a household were to erroneously report large quantities of foods entering the household that were for resale rather than home consumption, or were to report on the entire harvest of a food rather than the amount eaten by household members over the recall period. It is important to carefully document the number and percentage of households dropped and the reasons. If a large percentage are dropped, it is possible that a calculation or data entry error has affected a large number of cases. For example, a metric weight conversion factor for one of the primary staples may have been entered incorrectly. These possibilities should be investigated. The highest percentage of cases dropped from the analysis among the AFINS project surveys was 2.5 percent, and the average was 1.0 percent.

It is prudent to examine the distribution of the final calculated variable to make sure it follows a regular shape, which is typically log-Normal (skewed to the right, with the right tail longer than the left). Figure 1 shows distributions of dietary energy acquisition per capita for eight of the AFINS project countries. Note that, as discussed in Chapter 2, the range of the values is much wider than would be expected for household calorie intakes per capita, as measured in household food consumption surveys. The minimums, sometimes zero, are obviously far below what is possible for human survival. The maximums are far above what a person could possibly eat in one day, as expected.

An example STATA program for calculating daily household energy acquisition is given in Appendix 10.

Note that there may be some households that do not show up at all in the food data files because they reported no food acquisitions over the survey reference period. This is indeed a legitimate situation, because households that rely on purchases for their food may simply not have purchased any food during the survey reference period, instead relying on stocks accumulated before the period. These households should be merged back into the working data file and assigned zero energy acquisition.

If the price-per-calorie method of estimating calories from foods consumed outside the home is used rather than the more direct method recommended in the first section of Chapter 3, a separate analysis of this source of energy must be undertaken and the energy values from in-home and outside-the-home sources added together. Appendix 11 discusses how this method is implemented. 
Figure 1-Distributions of household calorie acquisition per capita for eight AFINS project countries
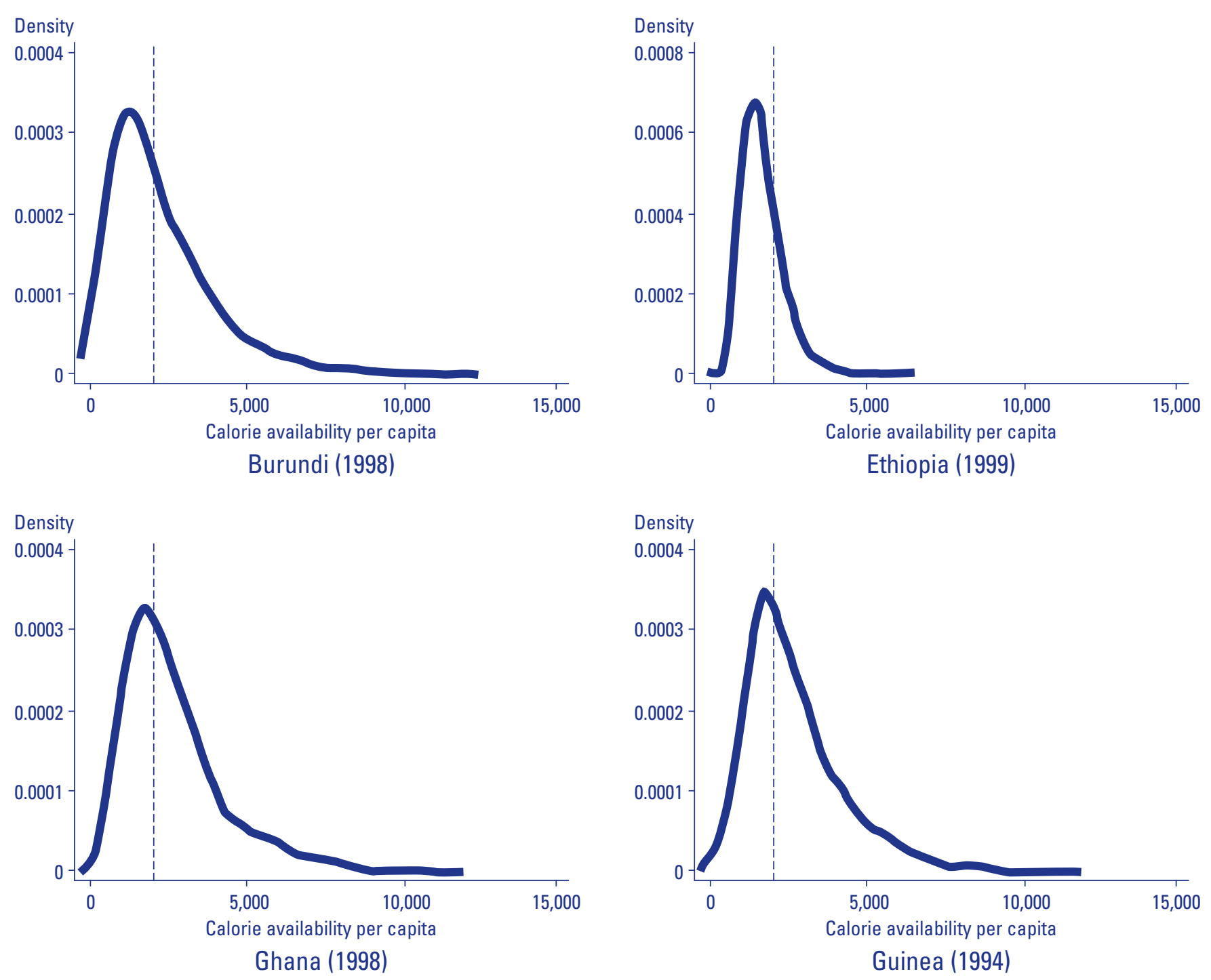

Sources: Smith and Subandoro (2005) and Smith, Alderman, and Aduayom (2006). Note: The vertical lines indicate the average per capita dietary energy requirement for light activity for each country.

72 Measuring Food Security Using Housebold Expenditure Surveys 

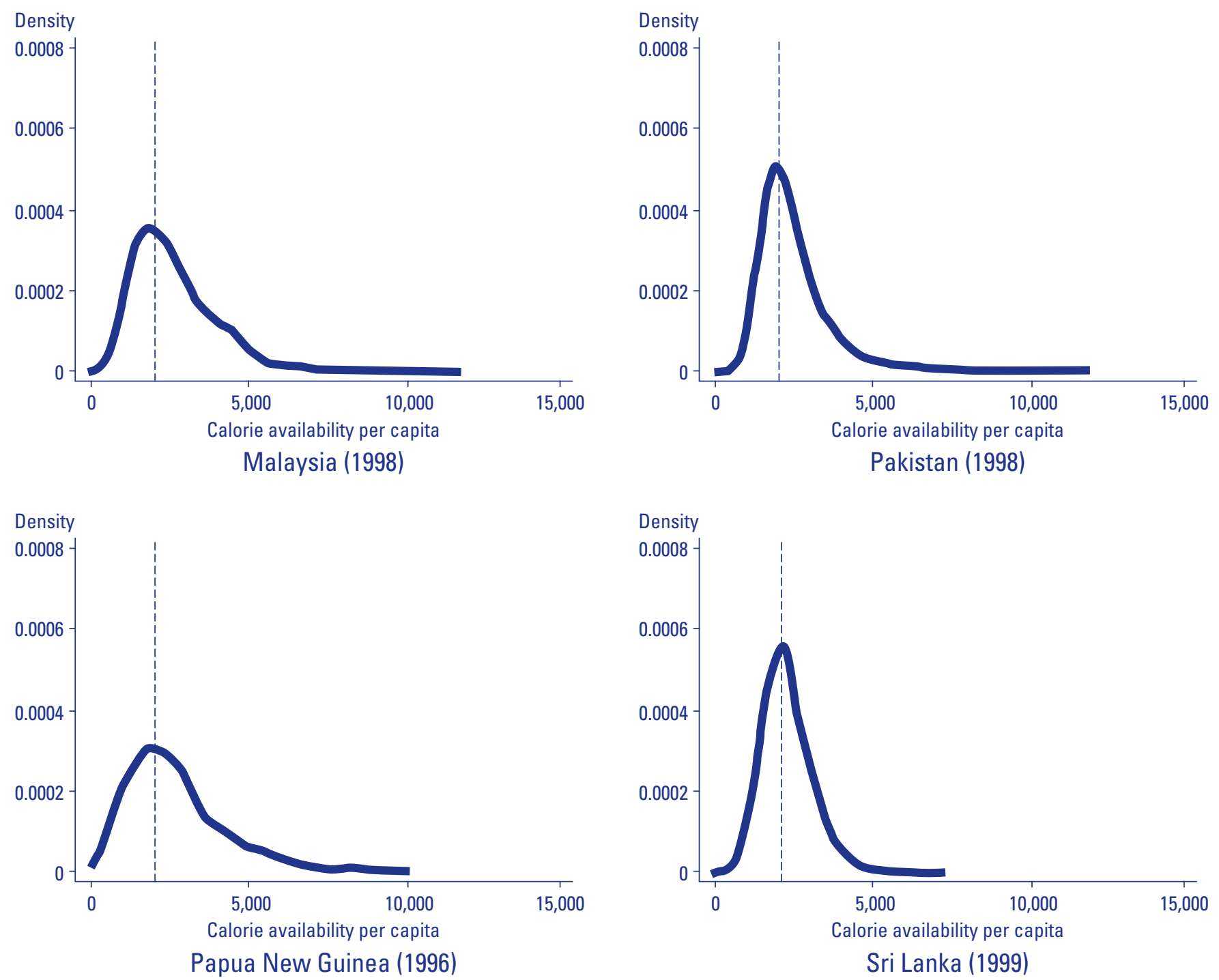


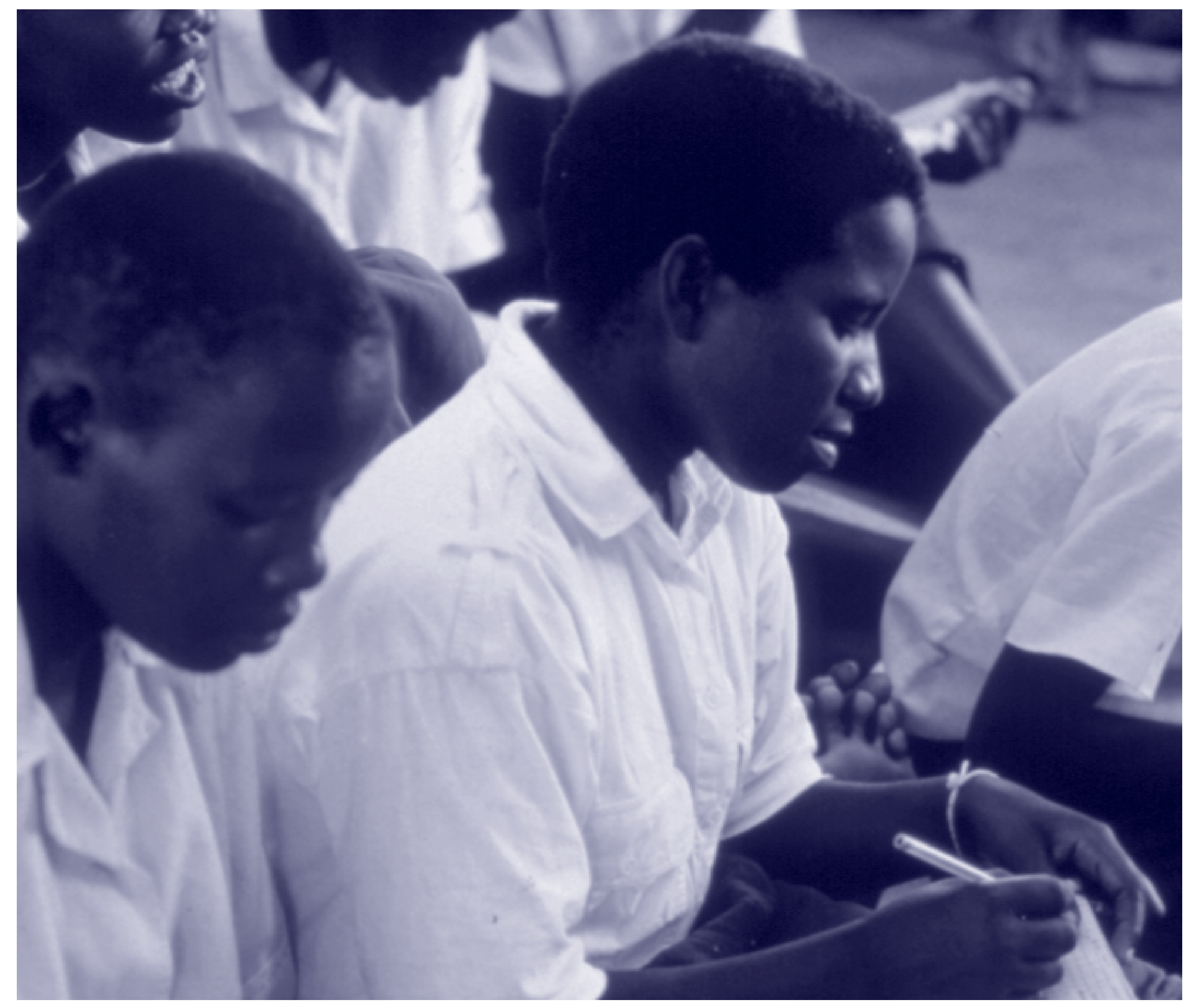




\section{Calculating the Indicators}

0 ce data processing and cleaning have been done, it is

straightforward to calculate the indicators listed in Table 2. Here we lay out how to calculate the household-level measures underlying the indicators and then how to calculate the population and population subgroup-level indicators. For the latter, it is as always important to take into account the sampling design specific to each survey. This usually involves specifying the sample strata, primary sampling units, and sampling weights assigned to each household. In STATA 8.0, it entails using the "svy" commands specially designed to take into account survey design. ${ }^{24}$ The STATA commands for doing so for the mean of a variable (for example, "kcal_pc") are as follows:

$$
\begin{aligned}
& \text { svyset [pweight=samplewt], psu(psu) strata(strata) } \\
& \text { svymean kcal_pc, }
\end{aligned}
$$

where the sampling weights are referred to as "samplewt," the strata as "strata," and the primary sampling units as "psu." The command for calculating the means for subgroups of a population, for example, geographic regions, is

$$
\text { svymean kcal_pc, by(region). }
$$

Table 8 contains national values of the indicators (with the exception of quantities of specific foods consumed) for the 20 AFINS project countries to be used for reference and comparison.

\section{DAILY FOOD ENERGY CONSUMPTION PER CAPITA}

The household-level measure underlying this indicator is daily household food energy availability per capita. It is calculated by dividing each household's daily food energy availability by the number of household members. The survey design corrected mean of the household-level values of this variable is then the population- (or population subgroup-) level indicator desired.

\section{Percentage of Households OR PEOPLE THAT ARE FOOD ENERGY DEFICIENT}

The household-level measure underlying this indicator is a dummy variable equal to one if a household's daily energy availability is less than its daily energy requirement and zero otherwise. The steps for calculating the variable are as follows:

1. Bring in the data file containing total daily household energy acquisition.

2. Merge the data file containing total daily energy requirements for each household with the working data file, matching cases by household.

${ }^{24}$ Note that the syntax will differ for STATA Versions 9.0 and above. That for STATA 8.0 is based on the Taylor series linearization approach of dealing with sample errors in multistage samples, whereas the higher versions can specifically deal with jackknife or balanced replication approaches as well. Note also that CS-Pro or IMPS can also be used to take complex survey designs into account in data analysis. 
Table 8. Food security indicators calculated from household expenditure survey data collected in 20 developing countries

\begin{tabular}{ll|l} 
& \multicolumn{2}{c}{ Diet quantity } \\
\cline { 2 - 3 } & $\begin{array}{c}\text { Daily food energy } \\
\text { consumption per } \\
\text { capita (kilocalories) }\end{array}$ & $\begin{array}{c}\text { Percentage of people } \\
\text { who are } \\
\text { food energy-deficient }\end{array}$ \\
\hline Sub-Saharan Africa & 1,592 & 74.8 \\
\hline Burundi & 1,648 & 76.4 \\
\hline Ethiopia & 2,328 & 51.4 \\
\hline Ghana & 2,510 & 45.1 \\
\hline Guinea & 2,579 & 43.9 \\
\hline Kenya & 1,664 & 76.7 \\
\hline Malawi & 2,059 & 60.3 \\
\hline Mozambique & 1,860 & 65.3 \\
\hline Rwanda & 1,967 & 60.2 \\
\hline Senegal & 2,454 & 43.9 \\
\hline Tanzania & 2,636 & 36.8 \\
\hline Uganda & 1,764 & 71.1 \\
\hline Zambia & & \\
\hline
\end{tabular}

\begin{tabular}{|c|c|c|}
\hline \multicolumn{2}{|c|}{ Diet quality } & $\begin{array}{l}\text { Economic } \\
\text { vulnerability }\end{array}$ \\
\hline $\begin{array}{l}\text { Diet diversity } \\
\text { (number of food } \\
\text { groups out of } 7)^{\mathrm{a}}\end{array}$ & $\begin{array}{l}\text { Percentage of food } \\
\text { energy from staples }\end{array}$ & $\begin{array}{l}\text { Percentage of } \\
\text { expenditures on food }\end{array}$ \\
\hline 4.5 & 62.3 & 76.1 \\
\hline 4.8 & 83.2 & 63.1 \\
\hline 5.8 & 66.9 & 61.4 \\
\hline 6.0 & 66.3 & 55.6 \\
\hline 5.4 & 61.8 & 74.8 \\
\hline 4.4 & 69.3 & 68.4 \\
\hline 4.2 & 77.3 & 67.9 \\
\hline 4.5 & 62.6 & 81.6 \\
\hline 5.9 & 55.5 & 61.0 \\
\hline 5.9 & 70.6 & 72.5 \\
\hline 4.4 & 69.4 & 59.3 \\
\hline 4.6 & 77.8 & 66.2 \\
\hline
\end{tabular}

76 Measuring Food Security Using Household Expenditure Surveys 
Table 8. Food security indicators calculated from household expenditure survey data collected in 20 developing countries (cont.)

\begin{tabular}{|c|c|c|c|c|c|}
\hline & \multicolumn{2}{|c|}{ Diet quantity } & \multicolumn{2}{|c|}{ Diet quality } & \multirow{2}{*}{$\begin{array}{c}\begin{array}{c}\text { Economic } \\
\text { vulnerability }\end{array} \\
\begin{array}{c}\text { Percentage of } \\
\text { expenditures on food }\end{array}\end{array}$} \\
\hline & $\begin{array}{l}\text { Daily food energy } \\
\text { consumption per } \\
\text { capita (kilocalories) }\end{array}$ & $\begin{array}{c}\text { Percentage of people } \\
\text { who are } \\
\text { food energy-deficient }\end{array}$ & $\begin{array}{l}\text { Diet diversity } \\
\text { (number of food } \\
\text { groups out of } 7)^{\mathrm{a}}\end{array}$ & $\begin{array}{l}\text { Percentage of food } \\
\text { energy from staples }\end{array}$ & \\
\hline \multicolumn{6}{|l|}{ Asia } \\
\hline Bangladesh & 2,100 & 53.7 & 6.1 & 82.4 & 60.1 \\
\hline India & 2,172 & 52.4 & 5.7 & 66.5 & 58.7 \\
\hline Indonesia & b & b & 5.6 & 59.6 & 60.6 \\
\hline Lao PDR & 2,471 & 34.7 & 4.4 & 88.4 & c \\
\hline Malaysia & 2,341 & 49.5 & 6.1 & 51.8 & 39.1 \\
\hline Pakistan & 2,422 & 44.1 & 6.5 & 56.2 & 50.9 \\
\hline Papua New Guinea & 2,330 & 49.2 & 5.1 & 61.7 & 54.7 \\
\hline Sri Lanka & 2,108 & 56.7 & 5.8 & 59.9 & 52.4 \\
\hline
\end{tabular}

Sources: Smith and Subandoro (2005) and Smith, Alderman, and Aduayom (2006).

Note: All values are corrected for survey sampling design.

The food groups are (1) cereals, roots, and tubers; (2) pulses and legumes; (3) dairy products; (4) meats, fish and seafood, and eggs; (5) oils and fats; (6) fruits; and (7) vegetables.

${ }^{6}$ Estimates deemed unreasonable and thus not reported (see Smith and Subandoro 2005).

"Total expenditures not available for calculating this measure. 
3. Create a variable equal to "1" if a household's energy acquisition is less than its energy requirement and " 0 " otherwise.

The population-level indicator is then calculated as the survey design corrected mean of the household-level values of the dummy variable multiplied by 100 .

When the goal is to measure the percentage of people who are food energy deficient, each individual included in the survey defined as a household member is assigned the same dummy variable outcome as his or her household. The population- (or population subgroup-) level indicator is then calculated as the survey design corrected mean of the individual values of the dummy variable multiplied by 100 . Note that the percentage of people who are food energy deficient will be higher than the percentage of households because households with low food energy availability tend to be larger.

\section{DIET DIVERSITY}

The household-level measure underlying this indicator, household diet diversity, is calculated by counting the number of foods or food groups from which each household acquired food over the survey reference period. The steps for doing so when food groups are used as the basis for calculation are as follows:

1. Bring in the data file containing the cleaned metric food quantities acquired by each household.

2. Assign a food group to each food.

3. Create a new data file containing the mean of the food group variable for each household (using the "collapse" command in STATA).
4. Create a variable with all cases equal to "1."

5. Count the number of food groups by creating a new data file containing the sum of the variable with all cases equal to " 1 " (using the "collapse" command in STATA).

The population- (or population subgroup-) level indicator is then the survey design corrected mean of the household-level values of this variable.

\section{PERCENTAGE OF FOOD ENERGY FROM STAPLES}

The household-level measure of this indicator is the percentage of food energy available from staples, that is, cereals, roots, and tubers. This measure is calculated as follows:

1. Bring in the data file containing the cleaned metric food quantities acquired by each household.

2. Drop the cases referring to nonstaple foods.

3. Transform each food quantity into its energy content using the edible portions and caloric conversion factors previously assembled.

4. Create a new data file containing the sum of the energy content variable, which gives the total energy in staple foods (using the "collapse" command in STATA)

5. Merge the data file containing total energy availability with the working data file, matching cases by household.

6. Calculate the percentage of energy from staples as

$$
\begin{aligned}
& \text { percentage of energy } \\
& \text { from staples }
\end{aligned}=\frac{\text { total energy from staples }}{\text { total energy from all foods }} \times 100 \text {. }
$$


The population- (or population subgroup-) level indicator is then the survey design corrected mean of the household-level values of this variable.

If the price-per-calorie method is used for calculating the energy in food acquired outside of the home, this measure can be based only on the foods acquired for consumption inside the home. The total energy available variable must then be recalculated to exclude the portion from food acquired outside the home.

\section{DAILY QUANTITIES OF SPECIFIC FOODS CONSUMED PER CAPITA}

The household-level measure of this indicator is the daily quantities of foods acquired. They are calculated as follows:

1. Bring in the data file containing the cleaned metric food quantities acquired by each household.

2. (Optional) If the edible quantities of foods are desired, multiply the food quantities by their edible portions previously assembled.

3. Organize the file in such a way that only the foods actually acquired by each household are listed. To account for the fact that a household acquired zero quantities of the other foods, transform the file in such a way that the foods become variables rather than cases and each household has a value for each food (using the "reshape" command in STATA) as follows:

reshape wide qty_g, i(hhid) j(foodcode).

4. Replace missing values for each food with zeros.
5. Divide the quantity of each food by the number of household members.

6. (Optional) If a food is listed in more than one form (for example, maize grain and maize flour) yet the desired quantity is for all of the food consumed, transform the processed quantities of the food into their "primary equivalent" values (see the fifth section of Chapter 4) before summing the quantities from the various forms. Note: If the edible quantities of foods are desired, the edible portion factors must be applied to the food items in their original forms before they are summed.

The population- (or population subgroup-) level indicator is then the survey design corrected mean of the household-level values of the per capita daily food quantity variables.

\section{PERCENTAGE OF EXPENDITURES ON FOOD}

The household-level measure of this indicator is the percentage of each household's total expenditures devoted to food. To calculate the measure, each household's daily expenditures on food must be matched at the household level with their total expenditures. The formula for calculating the percentage of expenditures on food is

$$
\text { percentage of expenditure on food }=\frac{\text { expenditure on food }}{\text { total expenditure }} \times 100 \text {. }
$$

The population- (or population subgroup-) level indicator is then the survey design corrected mean of the household-level values of this variable. 


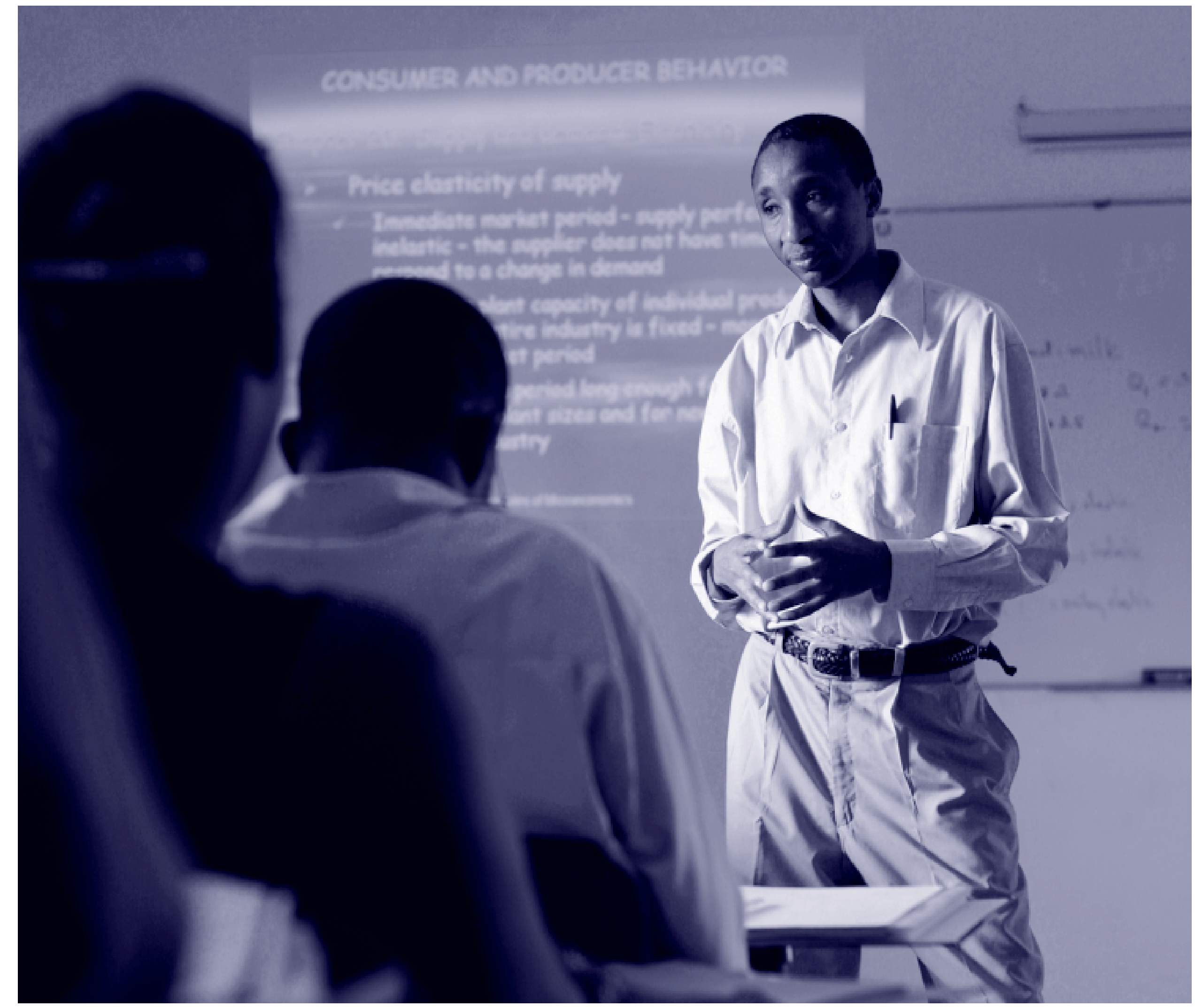




\section{Using the Indicators for Food Security Analysis}

$\mathrm{T}$ his chapter shows how to conduct the data analysis needed to understand the food insecurity problem of a population and design appropriate interventions to help the foodinsecure. A much broader set of data than those typically collected in household expenditure surveys is needed for a comprehensive food security analysis. These include data collected using qualitative techniques, a thorough review of previous literature, and analysis of available secondary data (Frankenberger, Mock, and Jere 2005). Further, they include additional quantitative data that can potentially be collected in household expenditure surveys, for example, anthropometric nutritional status data and information on food acquired through public assistance programs or those of nongovernmental organizations. Nevertheless, as will be seen in this chapter, a great deal can be learned from the data commonly collected in the surveys, which provide useful guidance.

A good understanding of the local context will help to formulate a set of relevant questions to be answered for any population. Here the answers to some general questions are explored to give examples of the types of analysis that can be undertaken, the ways data can be presented for analysis and reporting, and how to interpret results:

- Where are the food-insecure?

- What is the nature of the food insecurity problem (for example, diet quantity, diet quality, or both)?

${ }^{25}$ For more information on the surveys see Table 1.
- How does food insecurity change over time?

- What are the causes of food insecurity?

- Which are the most important foods in the diets of different sociodemographic groups?

Analysis of the answers to these questions is illustrated using data from household expenditure surveys conducted in Bangladesh, Lao People's Democratic Republic (PDR), Senegal, Tanzania, and Uganda. ${ }^{25}$

Table 9 provides general guidelines for interpreting the levels of the food security indicators featured in this manual and defined in Chapter 2. They take into account levels reported from across the globe, including those from developing, transitional, and developed countries.

\section{WHERE ARE THE FOOD-INSECURE? AN EXAMPLE FROM SENEGAL}

This question is one of the most basic to be answered to effectively target interventions toward those in need. Answering it entails calculating the food security indicators, as laid out in Chapter 6, for important geographical areas within the study population. These may include administrative regions, geophysical and agroclimatic zones, and rural or urban areas. It then entails entering the calculated numbers into tables and figures for interpretation. Box 10 discusses 


\section{Table 9. Guidelines for interpreting indicator levels}

\begin{tabular}{|c|c|}
\hline Indicator & Guideline for interpretation \\
\hline $\begin{array}{l}\text { Daily food energy consumption per capita } \\
\text { (kilocalories) }\end{array}$ & $\begin{array}{l}\text { The average requirement for light activity, at 2,050 kilocalories, serves as a benchmark for a very low } \\
\text { value of this indicator. Daily energy consumption per capita for developing countries, which exceeds } 3,400 \\
\text { kilocalories (von Braun et al. 2005), serves as an upper benchmark. Based on the above and the figures } \\
\text { given in Table } 8 \text { for the AFINS project countries, the guideline is } \\
\begin{array}{ll}3,000+: & \text { high } \\
2,500-3,000: & \text { medium } \\
2,050-2,500: & \text { low } \\
<2,050: & \text { very low }\end{array}\end{array}$ \\
\hline $\begin{array}{l}\text { Percentage of households or people that } \\
\text { are food energy-deficient }\end{array}$ & 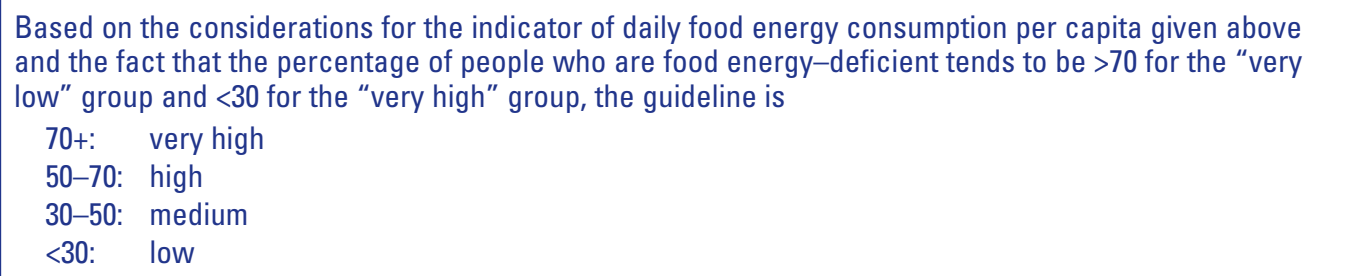 \\
\hline $\begin{array}{l}\text { Diet diversity (number of food groups } \\
\text { out of 7) }\end{array}$ & $\begin{array}{l}\text { Norms for ideal levels of diet diversity are not yet available. Swindale and Bilinksy (2005) suggest using } \\
\text { the diet diversity of the richest } 33 \text { percent of a population or the average diet diversity of the upper tercile } \\
\text { (highest } 33 \text { percent) as targets. Using the latter criterion and Table } 8 \text {, the guideline is } \\
6+\text { high } \\
\begin{array}{ll}4.5-6: & \text { medium } \\
<4.5: & \text { low }\end{array}\end{array}$ \\
\hline Percentage of food energy from staples & $\begin{array}{l}\text { Based on food supply data, in low-income developing countries the percentage of food energy derived } \\
\text { from staples averages around } 70 \text { percent; that of industrialized developed countries averages around } 30 \\
\text { percent (FAO 1996a). Among developing countries, a set classified by Bouis and Hunt (1999) as having } \\
\text { relatively good dietary quality receives } 55-70 \text { percent of their energy from staples. Based on the above } \\
\text { and Table } 8 \text {, the guideline is } \\
75+\text { : very high (very poor diet quality) } \\
60-75 \text { : high } \\
40-60 \text { : medium } \\
<40 \text { : low }\end{array}$ \\
\hline $\begin{array}{l}\text { Quantities of foods consumed daily } \\
\text { per capita }\end{array}$ & $\begin{array}{l}\text { No guideline available. Interpretation depends on the importance of each food in the population's diet and } \\
\text { specific policy goals. }\end{array}$ \\
\hline Percentage of expenditures on food & $\begin{array}{l}\text { The poorest households in the world spend more than } 75 \text { percent of their income on food. Households in the } \\
\text { richest countries, such as the United States and Canada, spend less than } 15 \text { percent of their expenditures } \\
\text { on food (COCA 2006; U.S. Department of Labor 2006). Based on the above and Table 8, the guideline is } \\
\text { 75+: very high (very vulnerable to food insecurity) } \\
65-75: \text { high } \\
\text { 50-65: medium } \\
<50 \text { : low }\end{array}$ \\
\hline
\end{tabular}

82 Measuring Food Security Using Household Expenditure Surveys 
some limitations on how finely disaggregated a breakdown by geographical area can be.

Table 10 presents the food security indicators for the major administrative regions and rural or urban areas of Senegal. While the country as a whole can be classified as having a high level of food insecurity when it comes to diet quantity, variation among its regions is great. The extremely low daily food energy consumption per capita for Zinguinchor, 1,430 kilocalories, contrasts sharply with the highest, 2,298, for Tambacouda. The corresponding prevalences of food energy deficiency for these regions are 83 and 42 percent, respectively. Noticeably, the capital, Dakar, has a very high prevalence of food energy deficiency, at 71 percent. Because the goal is often to reach the greatest number of those in need, in addition to calculating the percentage of people who are food energy deficient it may also be useful to calculate the numbers in each geographical area. This is because it is possible that the percentage is low in a very highly populated area, leading that area nevertheless to have a very high number of food-insecure people compared to the other areas. ${ }^{26}$

The great differences among Senegal's regions in terms of diet quantity are illustrated in Figure 2 using a bar graph of food energy deficiency prevalences. ${ }^{27}$ Note that estimates of food energy deficiency for Diourbel and Tambacouda are deemed to be imprecise; they have 95 percent confidence interval limits greater than 20 percent from the estimate, which would need to be pointed out in reporting (see Box 10). ${ }^{28}$
Box 10. Where Are the Food-Insecure? Deciding on the Level of Disaggregation

While it is tempting to calculate indicators for very

disaggregated geographical areas (for example, for subdistricts within a country's major administrative regions), there is a limit to how low one can go while still maintaining a reasonable degree of accuracy. The "standard error" and accompanying 95 percent confidence interval reported along with an estimate tells whether the estimate is likely to be very close to the actual population statistic. Specifically, the lower and upper limits of the 95 percent confidence interval provide the range within which one can be 95 percent sure that the population statistic lies. If the interval is "small," one can be assured that a reasonable degree of precision is achieved. In the AFINS project a reasonable degree of precision was assumed to have been achieved if the upper and lower limits of the 95 percent confidence interval were within 20 percent of the estimate. Normally the sample size of a survey is chosen to ensure that a particular breakdown can be used for key variables of interest with sufficiently narrow confidence intervals.

Alderman et al. (2002) discuss how finely disaggregated breakdowns or "maps" of indicators of poverty can be created by combining data from household expenditure surveys with those from census data.

${ }^{26}$ To do so, multiply the percentage of the people who are food energy-deficient by the population size of each region and divide by 100.

27 When there are many areas, it may be helpful to group them by the severity of the food insecurity problem and create a food security map (see, for example, Benson et al. 2002). This will help to reveal geographical patterns. For example, it may reveal that the regions in the northern part of a study area are generally more food-insecure than those in the south.

${ }^{28}$ The 95 percent confidence interval for the estimate of the prevalence of food energy deficiency in Diourbel is 63.9 to 73.8 . That for Tambacouda is 30.5 to 53.8 . 
Table 10. Food security indicators for administrative regions and rural versus urban areas of Senegal, 2001

\begin{tabular}{lc|c}
\cline { 2 - 3 } & \multicolumn{2}{c}{ Diet quantity } \\
\cline { 2 - 3 } & $\begin{array}{c}\text { Daily food energy } \\
\text { consumption per } \\
\text { capita (kilocalories) }\end{array}$ & $\begin{array}{c}\text { Percentage of people } \\
\text { who are } \\
\text { food energy-deficient }\end{array}$ \\
\hline National & 1,967 & 60.2 \\
\hline Regions & 1,803 & 70.6 \\
\hline Dakar & 1,430 & 82.7 \\
\hline Zinguinchor & 2,066 & {$[53.7]$} \\
\hline Diourbel & 1,800 & 64.7 \\
\hline Saint-Louis & 2,298 & {$[42.1]$} \\
\hline Tambacouda & 2,222 & 47.9 \\
\hline Kaolack & 1,994 & 59.6 \\
\hline Thies & 2,130 & 54.9 \\
\hline Louga & 2,183 & 48.9 \\
\hline Fatick & 1,948 & 62.0 \\
\hline Kolda & 2,065 & 54.3 \\
\hline Rural areas & 1,827 & 68.5 \\
\hline Urban areas & & \\
\hline
\end{tabular}

\begin{tabular}{c|c}
\hline \multicolumn{2}{c}{ Diet quality } \\
\hline $\begin{array}{c}\text { Diet diversity } \\
\text { (number of food } \\
\text { groups out of 7) }\end{array}$ & $\begin{array}{c}\text { Percentage of food } \\
\text { energy from staples }\end{array}$ \\
\hline 5.9 & 55.5 \\
\hline & \\
\hline 6.2 & 48.3 \\
\hline 5.2 & 59.1 \\
\hline 5.9 & 60.7 \\
\hline 6.0 & 49.0 \\
\hline 5.7 & 60.8 \\
\hline 5.8 & 64.5 \\
\hline 6.1 & 55.8 \\
\hline 5.9 & 48.8 \\
\hline 5.8 & 64.6 \\
\hline 5.5 & 60.5 \\
\hline 5.6 & 60.0 \\
\hline 6.3 & 49.7 \\
\hline
\end{tabular}

Economic

vulnerability

Percentage of expenditures on food

61.0

\begin{tabular}{l}
\hline 53.2 \\
\hline 55.4 \\
\hline 66.3 \\
\hline 65.3 \\
\hline 65.4 \\
\hline 64.1 \\
\hline 65.0 \\
\hline 63.5 \\
\hline 54.6 \\
\hline 64.3 \\
\hline 65.5 \\
\hline 55.0
\end{tabular}

Sources: Smith and Subandoro (2005) and Smith, Alderman, and Aduayom (2006).

Note: All values are corrected for survey sampling design. Square brackets indicate a 95 percent confidence interval whose limits are more than 20 percent from the value. 
The diet quality and economic vulnerability indicators, by contrast, show much less variation. As judged by the diet quality indicator, diet quality appears not to be a serious problem in any of the country's regions. Diet diversity ranges from 5.2 out of 7.0 food groups in Zinguinchor to 6.2 in Dakar. None of the regions falls into the "low" diet diversity category ( $<4.5$ food groups). Nevertheless, two regions, Kaolack and Fatick, fall well into the group with a "high" percentage of food energy from staples, suggesting that further investigation into the quality of the diets of households in this region is warranted, perhaps by examining food consumption patterns. As for economic vulnerability, most of the regions fall into the overall national classification of "medium" in terms of the percentage of household expenditures on food, suggesting that economic vulnerability is not severe.

Differences in food insecurity across rural and urban areas are fairly strong in the case of diet quantity. The average rural household consumes 238 more kilocalories of energy per capita than the average urban household. Consequently, the rate of food energy deficiency is higher in urban areas than in rural ones. Rural-urban differences in diet quality are also quite high, but here urban households seem to be doing better than rural households. The diet diversity indicator is 0.7 points higher for urban households, and urban households derive a lower percentage of their food energy from staples, 50 percent versus 60 percent in rural areas. Similarly, urban households are doing better than rural households when it comes to economic vulnerability; the average percentage of expenditures on food of urban households is 55 percent compared to 66 percent for rural households.

In examining differences in the levels of indicators among geographical areas, so far we have taken into account only the
Figure 2-Prevalences of food energy deficiency for the administrative regions of Senegal, 2001

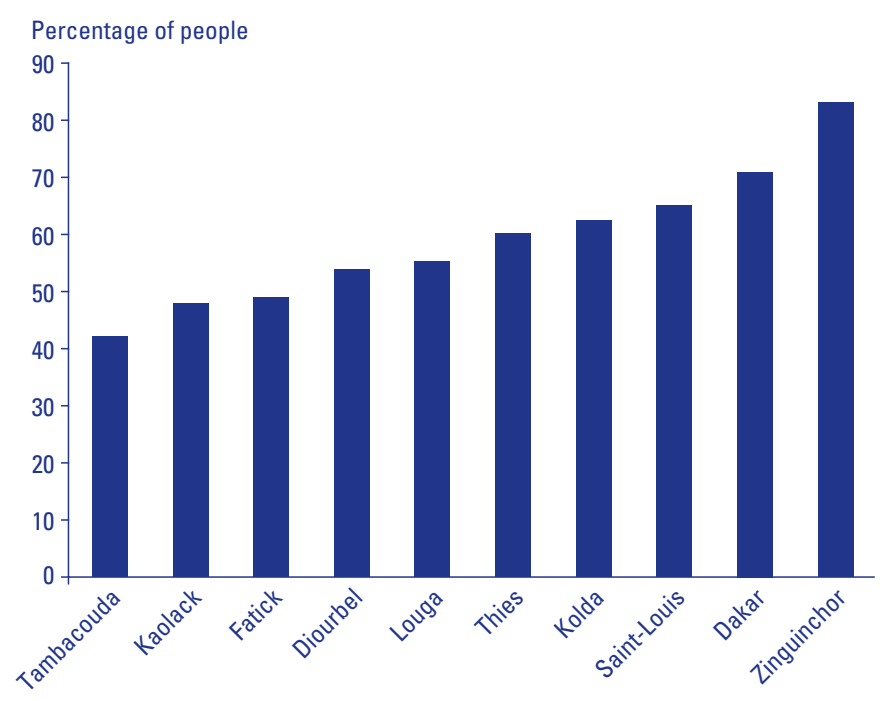

magnitude of such differences and their "practical significance" for food security policy. It is also important to conduct tests of the "statistical significance" of differences in indicators across population groups to determine whether the differences can be taken seriously. This is done through the use of a $t$-test, which tests whether the difference between the means of two subpopulations is different from zero. The command in STATA 8.0 for conducting the test, using the example of kilocalories per capita across rural and urban areas, is

$$
\text { ttest kcal_pc, by(urban). }
$$

Box 11 discusses how to interpret the " $p$-value" of a test, which helps us judge its statistical significance. For the differences between urban and rural areas reported in Table 10, $t$-tests reveal that they 


\section{Box 11. Statistical Significance and Practical Importance}

The term statistically significant means that the result of a test performed on data is probably true. Whether a test result is statistically

significant can be determined most easily by looking at the reported $p$-value of a test; $p$-values have the following interpretations:

\begin{tabular}{l|l|l}
$\boldsymbol{p}$-value & Statistical meaning & Interpretation \\
\hline$>0.10$ & $\begin{array}{l}\text { Statistically significant at greater than the } 10 \text { percent level. } \\
\text { (Test result has a greater than } 10 \text { percent chance of not being true.) }\end{array}$ & Not statistically significant \\
\hline 0.10 to 0.05 & $\begin{array}{l}\text { Statistically significant at the } 10 \text { percent level. } \\
\text { (Test result has a } 10 \text { percent to } 5 \text { percent chance of not being true.) }\end{array}$ & Low level of statistical significance \\
\hline 0.05 to 0.01 & $\begin{array}{l}\text { Statistically significant at the } 5 \text { percent level. } \\
\text { (Test result has a } 5 \text { percent to } 1 \text { percent chance of not being true.) }\end{array}$ & Statistically significant \\
\hline$<0.01$ & $\begin{array}{l}\text { Statistically significant at the } 1 \text { percent level. } \\
\text { (Test result has less than a } 1 \text { percent chance of not being true.) }\end{array}$ & High level of statistical significance
\end{tabular}

Note: Some analysts interpret any p-value greater than 0.05 as indicating that a test result is not statistically significant.

Even though a test result may be statistically significant, it is not necessarily practically important from a food security policy perspective. Practical importance is judged from prior knowledge of the particular variable of interest. It may also depend on the initial level of the variable in the population. For instance, if daily food energy consumption per capita is very high for two groups, say 3,500 kilocalories in urban areas and 3,300 in rural areas, a difference of 200 kilocalories would be considered small even if statistically significant. However, if energy consumption is very low, say 1,500 kilocalories in urban areas and 1,700 in rural areas, the difference would indeed be considered important from a food security perspective.

Sometimes differences that are significant from a practical standpoint are not statistically significant because of a small sample size. On the other hand, very small differences can be deemed highly statistically significant, especially when working with large samples. In these cases it is especially important to rely on logical reasoning rather than only on statistical testing to determine whether the difference should be taken seriously and acted on when it comes to planning interventions (Economist 2004). 
are statistically significant at the 1 percent level, that is, highly statistically significant.

\section{WHAT IS THE NATURE OF THE FOOD INSECURITY PROBLEM? AN EXAMPLE FROM LAO PDR}

It is crucial for policymakers and program planners to understand what kind of food insecurity problem they are trying to ameliorate. For instance, they will need to know whether they are dealing with what is primarily a diet quantity problem or a diet quality problem or both. Further, they will want to know whether there is enough food available to meet the needs of all citizens of a population, which will indicate whether the underlying problem is insufficient food availability or unequal distribution or both. These problems may need to be approached with different types of interventions.

Table 11 illustrates an analysis of the nature of the food insecurity problem in Lao PDR using data from that country. There diet quantity is a problem, but not a major issue. Daily food energy consumption per capita, at 2,471 kilocalories, is far above the average requirement for light activity, 2,050 kilocalories. Thirty-five percent of the population is estimated to be food energy deficient. This indicates that if the food available were to be distributed according to need, all people's needs could be met and that unequal distribution rather than insufficient food availability is a key issue. In contrast, diet quality appears to be a major problem. The average number of food groups consumed out of 7.0 is just 4.4, placing the country in the "low" diet quality category. The average percentage of energy derived from staples is a huge 88 percent, indicating that much of the population's food consumption comes from calorie-dense foods that are low in bioavailable protein and micronutrients, leaving people vulnerable to protein and micronutrient deficiencies. For the country as a whole, this suggests that while diet quantity needs to be addressed, diet quality should also be given priority.

Three regions in the country display the national pattern particularly strongly: Phongsaly, Huaphanh, and Xiengkhuang. In all of these regions the rate of food energy deficiency is near or below 20 percent, yet the diet diversity indicator is less than 4.0 and the percentage of food energy from staples is over 90 percent. The capital, Vientiane, presents a strong break with the national pattern. There the percentage of people who are food energy deficient is estimated to be quite high, at 67 percent. Yet the diet diversity score of 5.1 and the relatively low percentage of food energy from staples, 74 percent, indicate that the diet quality problem in the capital is not as big an issue as it is for the rest of the population.

An issue related to that of the nature of a population's food insecurity problem is whether an indicator that is simple and less costly to collect, for example, diet diversity, will give the same information as a more costly one, for example, food energy consumption per capita. If so, collecting data only on the less costly indicator would help reduce costs in future data collection efforts. The easiest way to find out is to see whether there is a strong association between the indicators by looking at their correlation coefficient. In STATA 8.0 the command for doing so (using the example of the above two variables) is:

$$
\text { pwcorr kcal_pc numfoodg, sig }
$$

where the "sig" modifier is used to present a $p$-value for statistical significance (see Box 11). 
Table 11. Diet quantity and quality food security indicators for Lao PDR, 2002

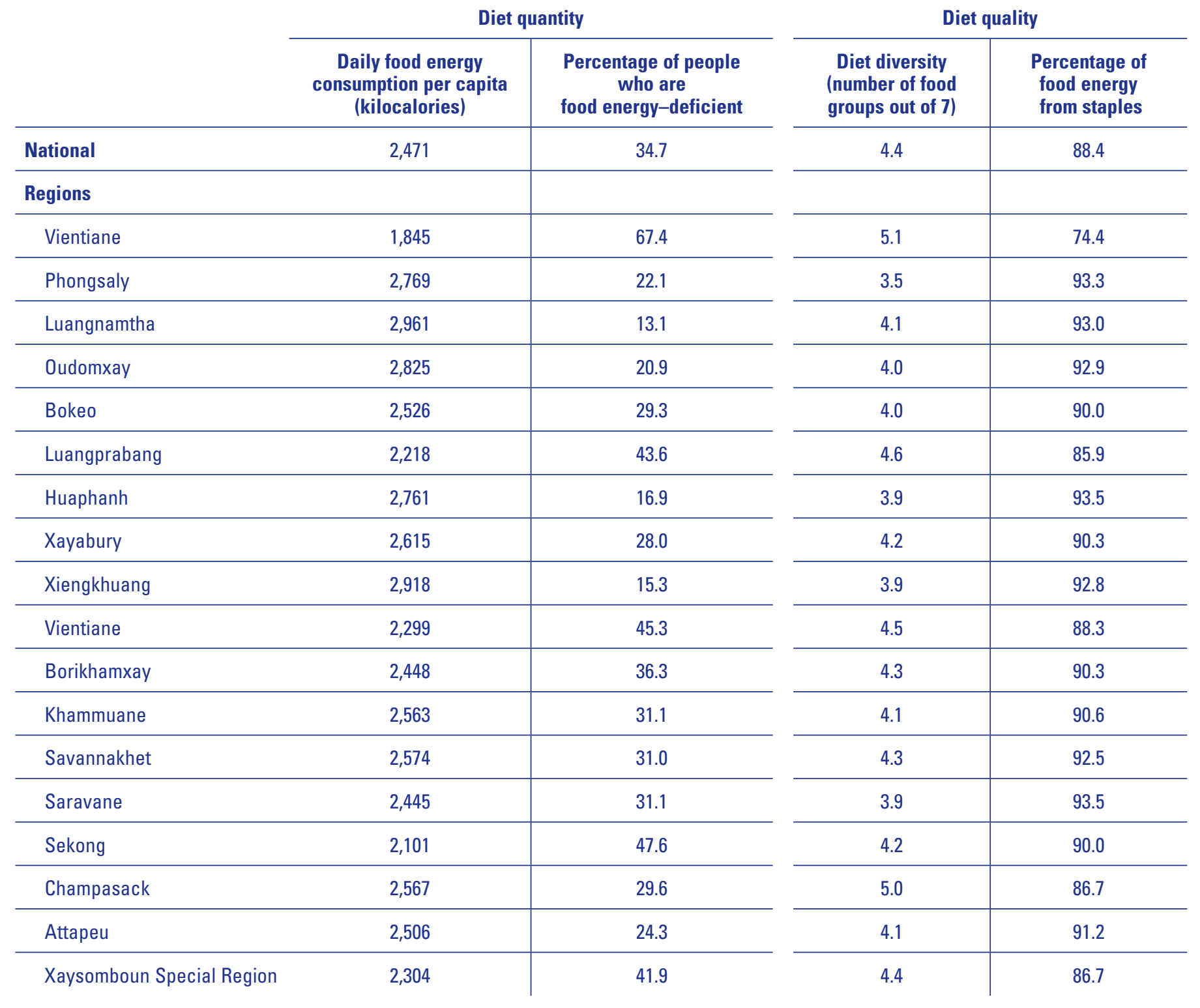

Note: All values are corrected for survey sampling design.

88 Measuring Food Security Using Household Expenditure Surveys 
In the case of the Lao PDR sample, the correlation coefficient between the two indicators is $-0.03(p=0.004)$. This suggests a statistically significant, near-zero, correlation. Using diet diversity as the sole indicator of food security will misrepresent the problem among this population. Hoddinott (2001) discusses this issue and other techniques for exploring associations between different indicators of food security.

\section{HOW DOES FOOD INSECURITY CHANGE OVER TIME? AN EXAMPLE FROM BANGLADESH}

Understanding how food insecurity changes over time is important for determining whether the situation is getting better or worse. It is also important for determining whether food insecurity is chronic or transitory. ${ }^{29}$ When food insecurity is analyzed at different points throughout a year, it can be used to find out if seasonal food insecurity is a problem..$^{30}$ With this information, analysts can inform policymakers as to when interventions are most needed.

Here we conduct an analysis of seasonal food security for Bangladesh in the year 2000. Figures 3 and 4 show how the diet quantity indicators changed over the period, month by month. From 2,100 kilocalories in January, daily food energy consumption per capita spiked to 2,300 by March, after which it declined fairly steadily to around 2,050 by September. It remained at this relatively low level throughout the last quarter of the year. This pattern is consistent with the fact that the rice crop contributing the most to the country's food energy supply, the Aman crop, is harvested in December (Del Ninno et al. 2001). This pattern is mirrored by the food energy deficiency prevalence measure, though it is not as distinct.

Diet quality, as viewed from the diet diversity indicator, followed a somewhat different pattern (Figure 4). From a low of 5.8, it rose steadily to peak almost one point higher in June. It had fallen almost to its original low by September, rising again slightly over the last quarter of the year. Upon examination of food consumption patterns in the country, the June peak coincided with a sharp increase in the percentage of households consuming fruits from January through June, followed by a sharp decline from July through November. The percentage of energy from staples varied only from 81 to 84 percent, remaining steady over the year. This reflects the fact that in Bangladesh persistent dependence on staples for dietary energy is very strong.

In sum, both of the diet quantity indicators, along with the diet diversity indicator, imply that food insecurity in Bangladesh is highest in the months of September through December, which precede the Aman rice harvest. While a consistent pattern for several years would

${ }^{29}$ Chronic vulnerability to food insecurity is defined as "persistence over time in the state of being vulnerable to food shortages." Transitory vulnerability is "a temporary inability to meet food needs or smooth consumption levels" (Frankenberger, Mock, and Jere 2005, p. 12).

${ }^{30}$ Note that unless panel data are collected (that is, data are collected for each household multiple times throughout a year), in order to conduct seasonal analysis as done in the example of this section, the primary sampling units (communities) containing the households must be randomly assigned over the months or seasons of the survey. 
Figure 3-Diet quantity indicators for Bangladesh,

by month, January-December 2000

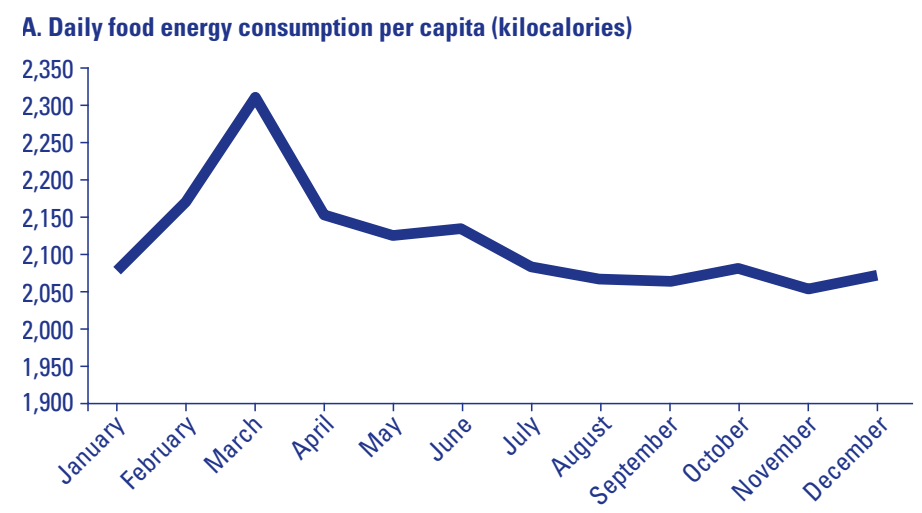

B. Prevalence of food energy deficiency (percentage of people)

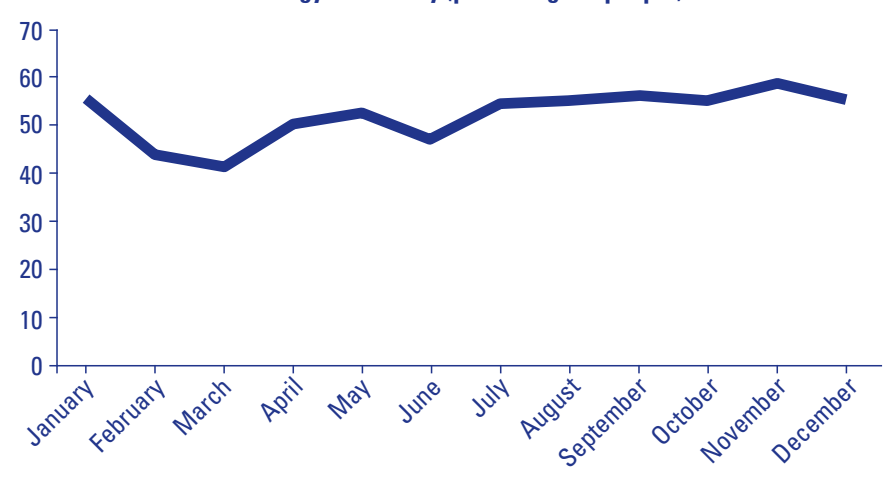

Figure 4-Diet quality indicators for Bangladesh,

by month, January-December 2000

\section{A. Diet diversity (number of food groups out of seven)}

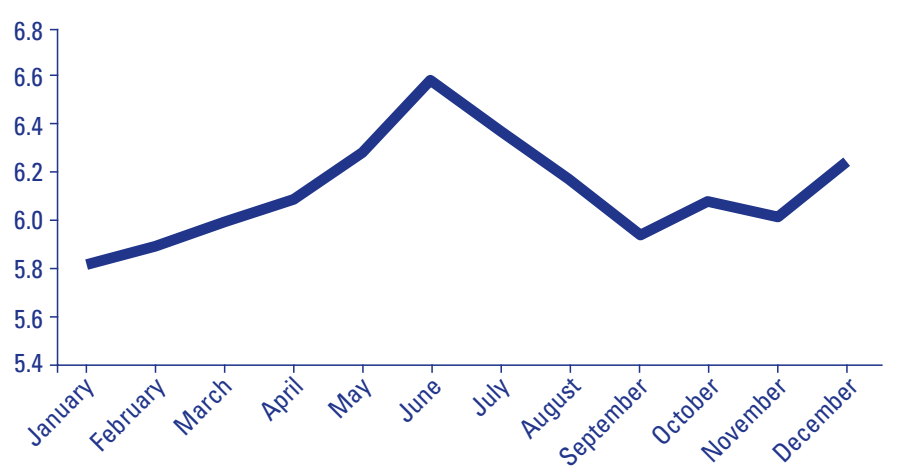

B. Percentage of energy from staples

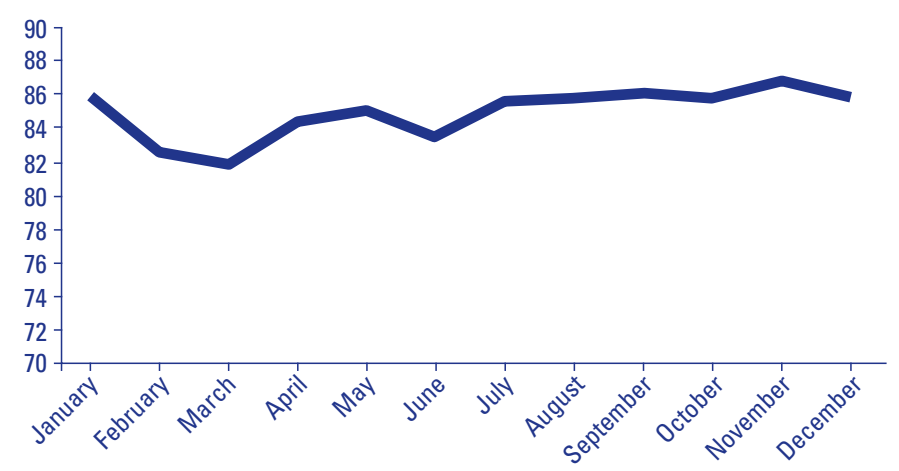


be needed to confirm this conclusion, it suggests that vulnerable population groups may need more support during this period.

\section{WHAT ARE THE CAUSES OF FOOD INSECURITY? AN EXAMPLE FROM UGANDA}

Understanding the causes of food insecurity is of primary importance in choosing appropriate interventions for addressing it. The causes are usually far ranging, from unfavorable climatic conditions, economic shocks, political instability, and HIV/AIDS through poverty and unequal distribution of food within households. A comprehensive analysis of the causes for a particular population must be undertaken in the context of this broad range and the particular issues affecting the population.

Household expenditure survey data can be used to understand only some of these causes, depending on the data collected. Here we show how a basic understanding of these causes can be achieved using simple descriptive analysis of quantitative data collected in Uganda in 1999 and looking at three variables: income, education, and gender of household head. Income is measured using households' total expenditures per capita. For analysis of the relationship between income and the food security indicators, households are classified into total expenditure quintiles, and the estimated values of the indicators are compared across the quintiles. ${ }^{31}$ Note that a more revealing analysis of causes can be undertaken using regression analysis in which the influence of any particular variable of interest is judged while controlling for other variables.

Table 12 presents the food security indicators by total expenditure quintile, education, and gender of household head for Uganda.

Household income has a very strong association with all of the food security indicators. Daily food energy consumption per capita jumps from a mean of 1,732 kilocalories among the poorest 20 percent of the population to 3,505 among the richest. The prevalence

${ }^{31}$ In the case of daily food energy consumption per capita, there will be populations for which the value of the lowest quintile is far below what is necessary for human survival and the value of the highest quintile is far above what it is possible for humans to consume in one day. This is partly due to the fact that in household expenditure surveys data are collected on foods acquired rather than consumed, which, as discussed in Chapter 2, can differ greatly because of the ability to store some foods. Because households in a randomly selected sample are equally likely to be drawing down their stocks as to be accumulating them, the mean of household energy acquisition will equal the mean of household energy consumption (the "error" is random). However, the acquisition-consumption difference presents some complications for analysis by income quintiles. Because total expenditure data are themselves based on the food acquisition data collected, the classification of households into quintiles will be incorrect. For example, a relatively poor household that happened to purchase a large quantity of grain over the survey reference period may be erroneously classified in the top quintile. Similarly, some relatively rich households that just happen to acquire no food over the reference period could be erroneously classified in the bottom quintile. Further, the top quintile will have an upwardly biased value of daily per capita food energy consumption, and the bottom quintile will have a downwardly biased value. The problem can be partially accounted for by basing the income quintiles on predicted total expenditures using predicted values from OLS regression with independent variables representing longer-term measures of household wealth, such as asset ownership, education, demographics, and so on, as in Smith, Alderman, and Aduayom (2006). This helps us classify households into their proper quintiles and gives more reasonable per capita calorie availabilities for the lower and upper quintiles. It is important to note that when the technique is applied to more quantitatively precise analysis of the relationship between income and energy availability, estimation of the "calorieincome elasticity," it is similar to instrumental variables estimation in the presence of measurement error. Subramanian and Deaton (1996) write that, due to the correlation between the error term and total expenditures (because energy availability and total expenditures are calculated from the same data), such estimation leads to downwardly biased estimates of the regression coefficient of total expenditures. Bouis and Haddad (1992) further discuss the issues. 
Table 12. Food security indicators for Uganda, by total expenditure quintile and education and gender of household head, 1999

\begin{tabular}{lc|c} 
& \multicolumn{2}{c}{ Diet quantity } \\
\cline { 2 - 3 } & $\begin{array}{c}\text { Daily food energy } \\
\text { consumption per } \\
\text { capita (kilocalories) }\end{array}$ & $\begin{array}{c}\text { Percentage of people } \\
\text { who are } \\
\text { food energy-deficient }\end{array}$ \\
\hline National & 2,636 & 36.8 \\
\hline Expenditure quintile & 1,732 & 67.4 \\
\hline 1 (poorest 20 percent) & 2,497 & 35.4 \\
\hline 2 & 2,892 & 23.3 \\
\hline 3 & 3,307 & 21.1 \\
\hline 4 & 3,505 & 16.3 \\
\hline 5 (richest 20 percent) & 2,808 & 35.4 \\
\hline Adult education & 2,812 & 32.8 \\
\hline No education & 3,129 & 34.7 \\
\hline Primary & 2,687 & 44.4 \\
\hline Secondary & 2,460 & \\
\hline Male-headed household & & \\
\hline Female-headed household & \multicolumn{2}{c}{} \\
\hline
\end{tabular}

\begin{tabular}{c|c}
\multicolumn{2}{c}{ Diet quality } \\
\hline $\begin{array}{c}\text { Diet diversity } \\
\text { (number of food } \\
\text { groups out of 7) }\end{array}$ & $\begin{array}{c}\text { Percentage } \\
\text { of food energy } \\
\text { from staples }\end{array}$ \\
\hline 4.4 & 69.4 \\
\hline \multicolumn{2}{|c}{} \\
\hline 3.7 & 72.1 \\
\hline 4.1 & 72.5 \\
\hline 4.5 & 71.5 \\
\hline 4.7 & 69.0 \\
\hline 5.3 & 60.5 \\
\hline & \\
\hline 3.8 & 71.1 \\
\hline 4.4 & 70.3 \\
\hline 5.1 & 65.0 \\
\hline 4.5 & 69.1 \\
\hline 4.2 & 70.1 \\
\hline
\end{tabular}

Economic vulnerability

Percentage

of expenditures on food

59.3

\begin{tabular}{l} 
\\
\hline 63.8 \\
\hline 62.8 \\
\hline 61.6 \\
\hline 58.1 \\
\hline 49.0 \\
\hline
\end{tabular}

Note: All values are corrected for survey sampling design. 
of food energy deficiency drops from almost 67 to 16 percent across the quintiles. In terms of diet quality, the diet diversity indicator rises just over one and a half points overall across the quintiles. Mimicking this pattern, the average percentage of energy from staples drops from 72 to 61 percent. As would be expected, as incomes increase, economic vulnerability declines: the average percentage of expenditures on food drops from 64 to 49 percent across the quintiles. Obviously, increasing income must be a key component of any strategy for reducing food insecurity in Uganda.

Turning to the role of education, among populations where there is insufficient food to meet energy needs, education may help improve diet quantity because it increases awareness of the need to ensure adequate food consumption to be productive and to increase the effectiveness of the management of household resources. However, where food consumption is in excess of food needs households may reduce their consumption in order to avoid obesity and the associated increased risk of chronic diseases. Education is expected to positively influence dietary quality because it brings greater awareness of and ability to understand nutrition knowledge and put it into practice (Rashid, Smith, and Rahman 2006).

Table 12 shows the food security indicators for three groups of households: those in which no adult member has any education, those in which at least one member has a primary education (but none has a secondary education), and those in which at least one member has a secondary education. Although there is very little increase in daily food energy consumption per capita across the "No education" and "Primary" groups, there is a fairly large increase, of 317 kilocalories, between the "Primary" and "Secondary" groups. Similarly, the percentage of households that are food energy deficient falls from 33 to 26 percent. With respect to diet quality, the diet diversity indicator shows substantial increases as one moves across the groups, from 3.8 to 4.4 and 5.1, a pattern mirrored by the indicator of percentage of energy from staples. The percentage of expenditures on food decreases by over 10 percentage points across the education groups. Together, these common patterns across the indicators suggest that education must also be a key component of strategies for reducing food insecurity in Uganda.

However, because households with higher incomes tend to be better educated, the apparent improvements in food security as education rises may really be attributable to income. Although regression analysis best addresses this problem of "confounding factors," it is possible to look into it by examining whether the education patterns observed at the population level are also found within each of the total expenditure quintiles. As Figure 5 shows, doing so gives us a great deal more information about the relationship between education and food security. It shows that in this population, food energy consumption tends to fall as education increases for all income groups except the first quintile — for which energy consumption is close to inadequate-where it rises slightly. This is not the case for diet diversity, however, which shows a clear tendency to increase with education across all income groups. In sum, this analysis shows that in Uganda education serves to reduce food energy consumption except among the very poorest households. But it serves to enhance diet quality.

Turning finally to gender of household head, in Uganda maleheaded households appear to have an advantage over female-headed households in terms of diet quantity. Daily food energy consumption per capita is 227 kilocalories higher in male- than in female-headed 
Figure 5-Daily food energy consumption and diet diversity in Uganda, by total expenditure quintile and adult education, 1999

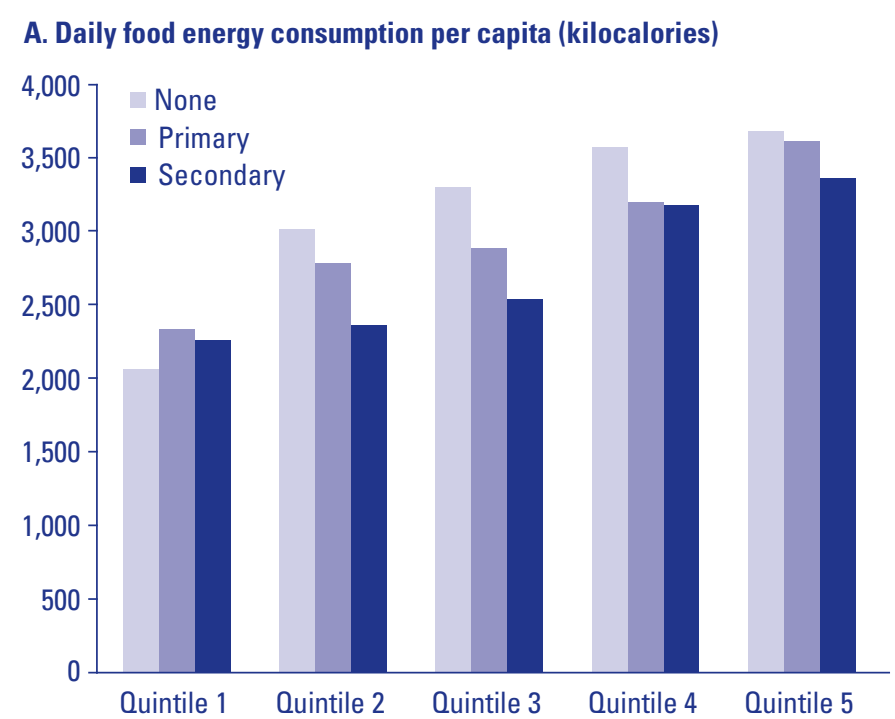

B. Diet diversity (number of food groups out of seven)

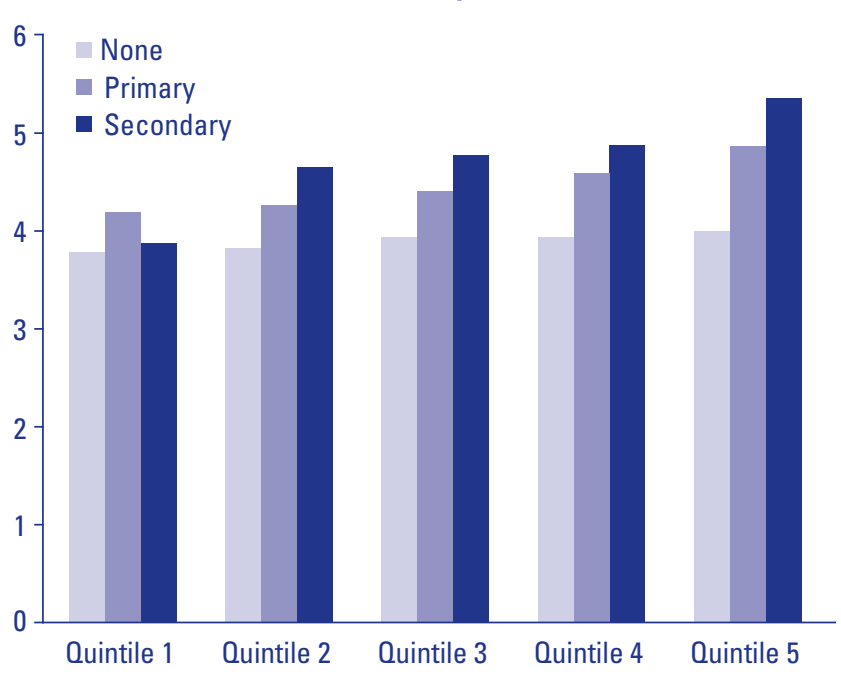

households. The percentage of people who are food energy deficient (which takes into account the different caloric requirements of households with different age-sex compositions) rises from 35 to 44 percent over the two groups. Diet quality is also higher in male-headed households, but both of the indicators suggest that this is only slightly so, as is the case for the economic vulnerability indicator. Note that the differences are highly statistically significant ( $p=0.000$; see Box 11) even though they are not practically significant from a food security perspective. Overall, the data suggest that in Uganda, female headship is an important determinant of households' dietary quantity but not of their dietary quality or economic vulnerability to food insecurity.

\section{WHICH ARE THE MOST IMPORTANT FOODS IN THE DIETS OF DIFFERENT SOCIODEMOGRAPHIC GROUPS? AN EXAMPLE FROM TANZANIA}

This final question is important for designing food security policies focused on improving or altering the consumption of specific foods. In doing so, it is critical to know which population groups consume which foods, how much they consume, and how important the foods are in their overall diet.

Table 13 looks at daily consumption of food staples per capita in Tanzania, a country in which nearly 50 percent of households are food energy deficient and the percentage of energy from food staples is quite high, at 71 percent (see Table 8). For the country as a whole, the staple food most highly consumed is maize, of which nearly 400 grams a day per person is consumed. The second most important staple is cassava, followed by rice, plantain bananas, sorghum, and sweet potatoes. 
Table 13. Consumption of food staples in Tanzania (grams per capita per day)

\begin{tabular}{|c|c|c|c|c|c|c|c|c|c|c|c|}
\hline & 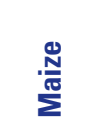 & $\begin{array}{l}\text { 芯 } \\
\frac{\stackrel{\Xi}{3}}{3}\end{array}$ & : & $\begin{array}{l}\text { E } \\
\text { 흫 } \\
\text { 心 }\end{array}$ & $\frac{\text { ప }}{\bar{E}}$ & 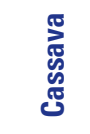 & 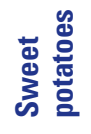 & 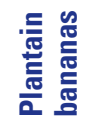 & 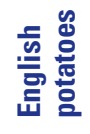 & 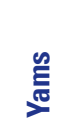 & $\frac{n}{\text { t๊ }}$ \\
\hline National & 381.7 & 16.2 & 68.7 & 40.4 & 4.0 & 214.4 & 33.7 & 52.9 & 13.0 & 6.3 & 5.9 \\
\hline \multicolumn{12}{|l|}{ Regions } \\
\hline Dodoma & 447.9 & 8.4 & 28.7 & 198.4 & 3.9 & 11.1 & 18.0 & 2.2 & 8.2 & 0.5 & 3.3 \\
\hline Arusha & 397.2 & 24.8 & 44.0 & 2.7 & 5.1 & 10.0 & 15.3 & 45.4 & 23.3 & 0.1 & 1.0 \\
\hline Kilimanj & 213.3 & 12.3 & 74.1 & 0.2 & 5.3 & 94.9 & 5.4 & 209.7 & 16.6 & 6.6 & 1.4 \\
\hline Tanga & 378.5 & 24.1 & 45.9 & 1.2 & 0.6 & 252.1 & 21.3 & 55.8 & 22.7 & 18.7 & 4.2 \\
\hline Morogoro & 361.5 & 14.8 & 142.6 & 4.8 & 1.0 & 64.5 & 18.8 & 57.6 & 6.7 & 12.4 & 7.2 \\
\hline Pwani & 390.5 & 24.9 & 121.6 & 1.8 & 1.2 & 203.1 & 14.4 & 20.3 & 4.3 & 1.7 & 10.6 \\
\hline Dar es Salaam & 183.0 & 43.0 & 126.0 & 0.2 & 1.3 & 20.9 & 9.8 & 14.3 & 15.1 & 1.9 & 27.7 \\
\hline Lindi & 295.7 & 15.1 & 113.6 & 58.8 & 1.4 & 493.2 & 13.2 & 30.2 & 2.0 & 3.5 & 6.4 \\
\hline Mtwara & 336.9 & 15.4 & 85.3 & 25.5 & 4.3 & 536.4 & 10.5 & 18.0 & 3.0 & 6.8 & 8.5 \\
\hline Ruvuma & 421.9 & 9.1 & 78.9 & 10.8 & 7.8 & 574.5 & 50.2 & 15.5 & 4.3 & 2.7 & 2.4 \\
\hline Iringa & 549.5 & 25.3 & 52.9 & 4.4 & 8.6 & 29.5 & 28.0 & 8.2 & 57.1 & 2.1 & 5.5 \\
\hline Mbeya & 432.3 & 18.7 & 80.1 & 2.0 & 5.6 & 51.1 & 44.1 & 76.7 & 26.3 & 14.4 & 6.5 \\
\hline Singida & 261.5 & 24.5 & 37.0 & 218.9 & 7.9 & 7.3 & 15.8 & 2.7 & 5.7 & 0.0 & 2.6 \\
\hline Tabora & 647.2 & 12.1 & 81.3 & 14.1 & 3.0 & 102.8 & 41.4 & 5.7 & 1.8 & 0.2 & 6.9 \\
\hline Rukwa & 584.6 & 5.2 & 22.9 & 8.8 & 6.3 & 307.4 & 19.8 & 5.2 & 8.8 & 0.4 & 0.8 \\
\hline Kigoma & 341.3 & 6.9 & 40.7 & 8.1 & 5.8 & 404.1 & 64.7 & 43.9 & 6.8 & 23.3 & 1.9 \\
\hline Shinyang & 561.3 & 9.1 & 74.0 & 137.8 & 1.4 & 51.7 & 74.7 & 2.0 & 1.5 & 0.9 & 5.3 \\
\hline Kagera & 179.0 & 4.5 & 26.2 & 12.6 & 4.4 & 304.3 & 58.1 & 322.0 & 16.2 & 22.7 & 2.8 \\
\hline Mwanza & 400.0 & 10.1 & 66.8 & 18.9 & 2.6 & 543.3 & 58.0 & 4.0 & 1.9 & 0.7 & 2.1 \\
\hline Mara & 197.5 & 9.8 & 34.0 & 77.7 & 5.7 & 791.0 & 56.7 & 14.3 & 2.8 & 0.4 & 3.1 \\
\hline Rural areas & 405.0 & 11.2 & 57.4 & 50.4 & 4.3 & 255.6 & 36.4 & 60.1 & 11.4 & 7.2 & 3.5 \\
\hline Urban areas & 297.0 & 34.5 & 109.9 & 4.1 & 3.0 & 65.0 & 24.2 & 26.9 & 18.6 & 2.9 & 14.3 \\
\hline \multicolumn{12}{|l|}{ Total expenditure quintile } \\
\hline 1 & 253.1 & 2.4 & 20.0 & 51.2 & 2.0 & 191.5 & 29.7 & 25.8 & 5.2 & 3.6 & 1.6 \\
\hline 2 & 349.5 & 6.2 & 41.9 & 40.3 & 3.2 & 241.6 & 32.8 & 53.7 & 9.8 & 6.5 & 2.9 \\
\hline 3 & 423.2 & 14.4 & 71.6 & 42.1 & 3.6 & 243.3 & 39.8 & 67.3 & 13.5 & 7.8 & 5.4 \\
\hline 4 & 477.0 & 25.8 & 110.5 & 26.0 & 6.4 & 214.1 & 39.9 & 80.5 & 18.7 & 10.4 & 9.9 \\
\hline 5 & 608.0 & 64.5 & 197.2 & 28.1 & 9.0 & 173.5 & 29.7 & 65.8 & 32.4 & 5.2 & 17.9 \\
\hline Male-headed household & 380.8 & 15.8 & 67.2 & 37.8 & 3.8 & 218.9 & 32.2 & 51.3 & 12.7 & 6.3 & 5.8 \\
\hline Female-headed household & 384.5 & 17.6 & 73.7 & 49.1 & 4.9 & 199.5 & 38.8 & 58.1 & 14.0 & 6.4 & 6.0 \\
\hline
\end{tabular}


Figure 6-Consumption of maize, rice, sorghum, and

cassava in Tanzania, by income quintile, 2000

\section{Grams per capita per day}

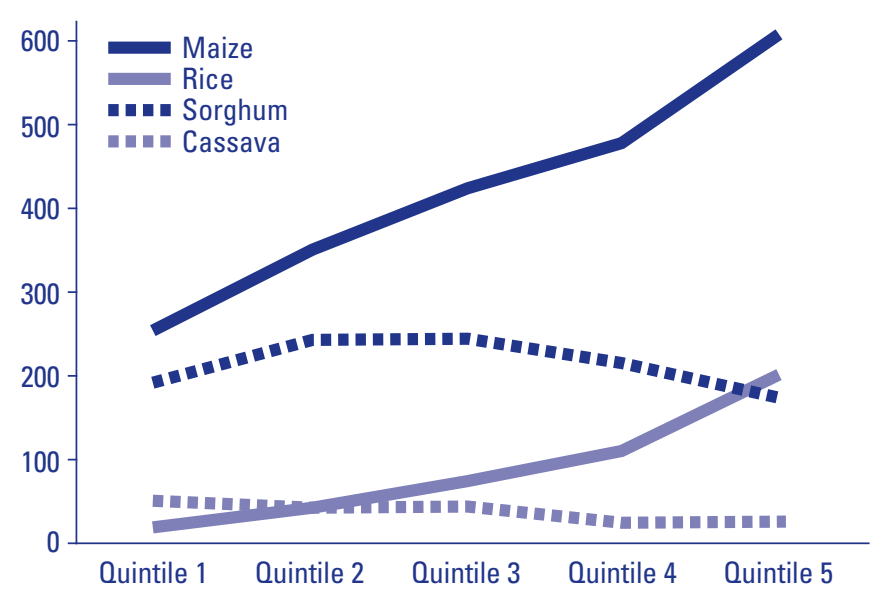

The national pattern does not fit for many of the country's regions, however. For example, in Kagera cassava and plantain bananas far surpass maize as the primary food staples. The average household in the region consumes around 300 grams per person per day of both cassava and plantain bananas, but only 179 grams of maize. In Mara, households are highly dependent on cassava as a staple food, consuming almost 800 grams per person per day. There are also strong differences across urban and rural areas, with rural households consuming around 400 grams of maize per person per day compared to 300 for urban households. Rural households also consume substantially more cassava than urban households. Conversely, urban households tend to consume more rice than rural households, around 100 grams compared to 50.

As is clear from the consumption statistics for the total expenditure quintiles, maize is an important food staple for all income groups, as is cassava. However, sorghum consumption declines as income increases (see Figure 6). This staple is important only in the diets of the poor. Cassava is also, relatively speaking, more important in the diets of the poor than in those of the rich. Conversely, rice is an important food staple for the rich but not for the poor. Sorghum and plantain bananas appear to be consumed in somewhat larger amounts in female- than in maleheaded households. There is very little difference in the average consumption of the other staples across male- and female-headed households. 


\section{Appendix 1}

\section{Food Groups with a Listing of the Most Common Foods}

\begin{tabular}{|c|c|c|}
\hline Food groups & Most common foods & Forms in which available \\
\hline Cereals & Wheat, rice, maize, barley, oats, millet, sorghum, teff & $\begin{array}{l}\text { Whole grains, popped grains, puffed grains, meals, } \\
\text { flours, pastas (macaroni, spaghetti, vermicelli, couscous), } \\
\text { commercial baked goods (bread, buns, cakes, biscuits, } \\
\text { breakfast cereals, cookies, pastries) }\end{array}$ \\
\hline $\begin{array}{l}\text { Roots, tubers, and } \\
\text { plantains }\end{array}$ & $\begin{array}{l}\text { Potatoes, sweet potatoes, yams, cocoyams, cassava, taro, } \\
\text { sago, plantain bananas }\end{array}$ & Fresh, dry, flour, meal, fried chips \\
\hline $\begin{array}{l}\text { Pulses, legumes, nuts, } \\
\text { and seeds }\end{array}$ & $\begin{array}{l}\text { Beans, dry peas, lentils, chickpeas, pigeon peas, green } \\
\text { or black gram, groundnuts (peanuts), coconuts, cashews, } \\
\text { almonds, walnuts, sesame seeds, sunflower seeds, soybeans }\end{array}$ & $\begin{array}{l}\text { Whole, shelled or unshelled, mature or immature (for } \\
\text { coconuts), paste, flour, sauce, curd }\end{array}$ \\
\hline Vegetables & $\begin{array}{l}\text { Roots, bulbs, and tubers: beets, carrots, kohlrabi, leeks, } \\
\text { onions, garlic, okra, radishes } \\
\text { Leafy vegetables: bean sprouts, beet greens, brussels } \\
\text { sprouts, cabbage, cassava leaves, celery, kale, lettuce, } \\
\text { spinach, parsley, pumpkin leaves, sweet potato leaves, } \\
\text { collard greens } \\
\text { Others: tomatoes, broccoli, cauliflower, cucumbers, eggplant, } \\
\text { sweet corn, pumpkins, squash, gourds, fresh peppers, fresh } \\
\text { beans, fresh peas, mushrooms, chives, bamboo shoots, } \\
\text { asparagus, local indigenous fruit vegetables }\end{array}$ & Fresh, dry, canned, powdered, sauce, paste \\
\hline Fruits & $\begin{array}{l}\text { Sweet bananas } \\
\text { Citrus fruits: oranges, tangerines, grapefruit, lemons, limes } \\
\text { Fat-rich fruits: avocados, olives } \\
\text { Others: apples, apricots, berries, cherries, guavas, mangoes, } \\
\text { melons, papayas, passion fruit, peaches, pears, pineapples, } \\
\text { plums, jack fruit, watermelons, grapes, durian, star fruit, local } \\
\text { indigenous fruits }\end{array}$ & Fresh, dry \\
\hline Meats & $\begin{array}{l}\text { Beef, pork, goat, mutton, buffalo, camel, horse, rabbit, "game } \\
\text { meat," "wild meat," chicken, duck, goose, pigeon, turkey, } \\
\text { Guinea hen, insects }\end{array}$ & $\begin{array}{l}\text { With or without bone, offal (liver, kidney, brain, heart), various } \\
\text { animal parts, bacon, ham, canned beef, sausages }\end{array}$ \\
\hline
\end{tabular}




\begin{tabular}{l|l|l} 
Food groups & Most common foods & Forms in which available \\
\hline Fish and seafood & $\begin{array}{l}\text { Fresh fish: salmon, trout, herring, mackerel, cod, haddock } \\
\text { Shellfish: lobster, crawfish, crab, shrimp, oysters, clams, } \\
\text { mussels }\end{array}$ & Salted, smoked, dried, canned (in oil or not) \\
\hline $\begin{array}{l}\text { Milk and dairy } \\
\text { products }\end{array}$ & $\begin{array}{l}\text { Liquid milk (cow, goat, sheep, buffalo, camel) (whole or } \\
\text { skimmed) }\end{array}$ & $\begin{array}{l}\text { Evaporated or condensed milk (sweetened or unsweetened), } \\
\text { powdered milk, cheese, cream, yogurt, ice cream, cottage } \\
\text { cheese, buttermilk, curd, infant formula }\end{array}$ \\
\hline Eggs & Hen eggs, duck eggs, goose eggs, turtle eggs & \\
\hline Oils and fats & $\begin{array}{l}\text { Oils: vegetable oils, peanut oil, palm oil } \\
\text { Fats: margarine, shortening, butter, ghee, lard, shea butter, } \\
\text { mayonnaise }\end{array}$ & $\begin{array}{l}\text { Alcoholic: industrial beers, wines, spirits; local beers, wines, } \\
\text { spirits } \\
\text { Nonalcoholic: fruit juices, soft drinks, coffee, tea }\end{array}$ \\
\hline Beverages & $\begin{array}{l}\text { Spices, salt, sugar, honey, syrups, molasses, jams, } \\
\text { marmalade, sugarcane, condiments (vinegar, ketchup, } \\
\text { mustard spread), chewing gum, chocolate, candy, baking } \\
\text { powder, baking soda }\end{array}$ &
\end{tabular}

Sources: AFINS project data sets (see Smith and Subandoro 2005 and Smith, Alderman, and Aduayom 2006) and United Nations (1989). Note: Foods listed are as acquired, before any preparation. 


\section{Appendix 2}

Local Units of Measure Used in 20 National Household Expenditure Surveys

\begin{tabular}{|c|c|c|}
\hline Food & Unit of measure & Country \\
\hline \multicolumn{3}{|l|}{ Cereal grains } \\
\hline \multirow[t]{12}{*}{ Maize } & Basket—small, medium, large & Burundi, Rwanda \\
\hline & Bowl & Burundi, Rwanda \\
\hline & Bucket & Burundi \\
\hline & Can & Mozambique \\
\hline & Cooking pot—small, medium, large & Burundi \\
\hline & Heap & Burundi \\
\hline & Margarine tin (Blueband) $-1 / 2,1$, or $2 \mathrm{~kg}$ & Uganda \\
\hline & Pan & Rwanda \\
\hline & Plate & Burundi, Rwanda \\
\hline & Sack-25, 50 , or $100 \mathrm{~kg}$ (original content unspecified) & Burundi \\
\hline & Sack—small, large & Rwanda \\
\hline & Various containers ("SIRVA," “metal," "plastic") & Rwanda \\
\hline \multirow[t]{12}{*}{ Maize, on cob } & American tin & Ghana \\
\hline & Basin & Rwanda \\
\hline & Basket & Ghana \\
\hline & Basket—small, medium, large & Rwanda \\
\hline & Bowl & Ghana, Rwanda, Guinea \\
\hline & Calabash & Guinea \\
\hline & Heap & Rwanda, Uganda \\
\hline & Plate & Rwanda \\
\hline & Sack -50 or $100 \mathrm{~kg}$ (original content unspecified) & Guinea \\
\hline & Sack—small, large & Rwanda \\
\hline & Unities & Uganda, Ghana, Rwanda \\
\hline & Various containers ("SIRVA," “metal," "plastic") & Rwanda \\
\hline
\end{tabular}




\begin{tabular}{|c|c|c|}
\hline Food & Unit of measure & Country \\
\hline \multicolumn{3}{|c|}{ Cereal grains (continued) } \\
\hline \multirow[t]{16}{*}{ Rice } & American tin, margarine tin & Ghana \\
\hline & Basket & Ghana \\
\hline & Basket—small, medium, large & Burundi \\
\hline & Bowl & Burundi, Ghana, Guinea \\
\hline & Calabash & Guinea \\
\hline & Can & Mozambique \\
\hline & Cooking pot—small, medium, large & Burundi \\
\hline & Cup & Burundi, Uganda \\
\hline & Glass & Mozambique \\
\hline & Large basin & Malawi \\
\hline & Packet & Uganda \\
\hline & Plate & Burundi \\
\hline & Plate_“Nsima plate” & Malawi \\
\hline & Sack—25-kg rice bag & Papua New Guinea \\
\hline & Sack-50 or $100 \mathrm{~kg}$ (original content unspecified) & Guinea \\
\hline & Sack—50 or $100 \mathrm{~kg}$ (original content unspecified) & Burundi \\
\hline \multirow[t]{9}{*}{ Sorghum and millet } & American tin, margarine tin & Ghana \\
\hline & Bowl & Ghana, Rwanda \\
\hline & Calabash & Uganda \\
\hline & Can & Mozambique \\
\hline & Finger & Ghana \\
\hline & Glass & Mozambique \\
\hline & Goblet & Rwanda \\
\hline & Margarine tin (Blueband)-1/2, 1, or $2 \mathrm{~kg}$ & Uganda \\
\hline & Plate & Rwanda \\
\hline
\end{tabular}

100 Measuring Food Security Using Household Expenditure Surveys 


\section{Food}

Unit of measure

Country

Cereal grains (continued)

\begin{tabular}{l|l|l}
\multirow{3}{*}{ Sorghum and millet (continued) } & Plate_“No. 10 plate," "relish plate" & Malawi \\
\cline { 2 - 3 } & Spoon & Rwanda \\
\cline { 2 - 3 } & Tin-“18-liter tin" & Uganda \\
\cline { 2 - 3 } & Various containers ("SIRVA," "metal," "plastic") & Rwanda \\
\hline \multirow{2}{*}{ Wheat } & Bowl & Rwanda \\
\cline { 2 - 3 } & Goblet & Rwanda \\
\cline { 2 - 3 } & Plate & Rwanda \\
\hline & Various containers ("SIRVA," "metal," "plastic") & Rwanda
\end{tabular}

Processed foods from cereal grains (not including flours)

\begin{tabular}{l|l|l} 
Bread (loaf) & Unities & Burundi, Mozambique, Pakistan, Uganda \\
\hline Buns & Unities & Pakistan \\
\hline Biscuits & Unities & Sri Lanka \\
\hline Cakes & Unities & Mozambique, Sri Lanka \\
\hline Cookies & Unities & Mozambique \\
\hline Pastries & Can & Mozambique \\
\cline { 2 - 3 } & Unities & Mozambique, Pakistan
\end{tabular}

\section{Roots and tubers}

Cassava

\begin{tabular}{|l|l}
\hline American tin & Ghana \\
\hline Bag_“minibag” or "maxibag” & Ghana \\
\hline Basin & Rwanda \\
\hline Basket & Burundi, Ghana \\
\hline Basket—small, medium, large & Burundi, Rwanda \\
\hline Bowl & Ghana, Guinea, Rwanda \\
\hline Bucket & Burundi \\
\hline Bundle & Burundi, Ghana
\end{tabular}




\begin{tabular}{|c|c|c|}
\hline Food & Unit of measure & Country \\
\hline \multicolumn{3}{|c|}{ Roots and tubers (continued) } \\
\hline \multirow[t]{14}{*}{ Cassava (continued) } & Calabash & Guinea \\
\hline & Cooking pot—small, medium, large & Burundi \\
\hline & Goblet & Rwanda \\
\hline & Heap & Burundi, Mozambique, Rwanda, Uganda \\
\hline & Log & Ghana \\
\hline & Pan & Rwanda \\
\hline & Plate & Burundi, Rwanda \\
\hline & Sack-25-kg rice bag & Papua New Guinea \\
\hline & Sack-25,50, or $100 \mathrm{~kg}$ (original content unspecified) & Burundi \\
\hline & Sack—50 or $100 \mathrm{~kg}$ (original content unspecified) & Guinea \\
\hline & Sack—small, large & Rwanda \\
\hline & Unities & Burundi, Ghana, Papua New Guinea, Rwanda \\
\hline & Van & Rwanda \\
\hline & Various containers (“SIRVA," "metal," "plastic”) & Rwanda \\
\hline \multirow[t]{8}{*}{ Plantain bananas } & Basket & Ghana \\
\hline & Bunch-small, medium, large & Uganda \\
\hline & Bundle & Ghana \\
\hline & Cluster & Uganda \\
\hline & Finger & Ghana \\
\hline & Heap & Uganda \\
\hline & Margarine tin & Ghana \\
\hline & Unities & Ghana \\
\hline \multirow[t]{4}{*}{ Potatoes } & Basin & Rwanda \\
\hline & Basket & Burundi \\
\hline & Basket—small, medium, large & Burundi, Rwanda \\
\hline & Bowl & Burundi, Rwanda \\
\hline
\end{tabular}

102 Measuring Food Security Using Household Expenditure Surveys 


\begin{tabular}{|c|c|c|}
\hline Food & Unit of measure & Country \\
\hline \multicolumn{3}{|c|}{ Roots and tubers (continued) } \\
\hline \multirow[t]{11}{*}{ Potatoes (continued) } & Bucket & Burundi \\
\hline & Can & Mozambique \\
\hline & Cooking pot—small, medium, large & Burundi \\
\hline & Heap & Burundi, Malawi, Mozambique, Rwanda, Uganda \\
\hline & Pan & Rwanda \\
\hline & Plate & Burundi, Rwanda \\
\hline & Sack-25-kg rice bag & Papua New Guinea \\
\hline & Sack-25, 50, or $100 \mathrm{~kg}$ (original content unspecified) & Burundi \\
\hline & Unities & Guinea, Malawi, Rwanda, Tanzania \\
\hline & Van & Rwanda \\
\hline & Various containers ("SIRVA," "metal," "plastic") & Rwanda \\
\hline \multirow[t]{15}{*}{ Sweet potatoes } & Bag_-"minibag" or "maxibag" & Ghana \\
\hline & Basin & Rwanda \\
\hline & Basket & Ghana \\
\hline & Basket—small, medium, large & Burundi, Rwanda \\
\hline & Bowl & Burundi, Ghana, Rwanda \\
\hline & Bucket & Burundi \\
\hline & Bundle & Ghana \\
\hline & Can & Mozambique \\
\hline & Heap & Burundi, Malawi, Mozambique, Rwanda, Uganda \\
\hline & Pan & Rwanda \\
\hline & Plate & Burundi, Rwanda \\
\hline & Sack-25-kg rice bag & Papua New Guinea \\
\hline & Sack-25, 50, or $100 \mathrm{~kg}$ (original content unspecified) & Burundi \\
\hline & Sack—small, large & Rwanda \\
\hline & Tuber & Ghana \\
\hline
\end{tabular}




\begin{tabular}{|c|c|c|}
\hline Food & Unit of measure & Country \\
\hline \multicolumn{3}{|l|}{ Roots and tubers (continued) } \\
\hline \multirow[t]{3}{*}{ Sweet potatoes (continued) } & Unities & Malawi, Rwanda \\
\hline & Van & Rwanda \\
\hline & Various containers (“SIRVA," “metal," "plastic”) & Rwanda \\
\hline \multirow[t]{17}{*}{ Taro, yams } & American tin & Ghana \\
\hline & Basin & Rwanda \\
\hline & Basket & Ghana \\
\hline & Basket—small, medium, large & Burundi, Rwanda \\
\hline & Bowl & Burundi, Ghana, Rwanda \\
\hline & Bucket & Burundi \\
\hline & Bundle & Ghana \\
\hline & Cooking pot—small, medium, large & Burundi \\
\hline & Heap & Rwanda \\
\hline & Milk pot & Rwanda \\
\hline & Pan & Rwanda \\
\hline & Plate & Burundi, Rwanda \\
\hline & Sack-25-kg rice bag & Papua New Guinea \\
\hline & Sack-25, 50 , or $100 \mathrm{~kg}$ (original content unspecified) & Burundi \\
\hline & Unities & Ghana, Guinea, Rwanda \\
\hline & Van & Rwanda \\
\hline & Various containers ("SIRVA," "metal," "plastic") & Rwanda \\
\hline \multicolumn{3}{|l|}{ Dried roots and tubers } \\
\hline Cassava, sweet potatoes & Heap & Mozambique, Uganda \\
\hline \multicolumn{3}{|c|}{ Flours from cereal grains, roots, and tubers } \\
\hline \multirow[t]{2}{*}{ Cassava, maize, millet } & $1 / 2$-liter cup & Uganda \\
\hline & American tin, margarine tin & Ghana \\
\hline
\end{tabular}

104 Measuring Food Security Using Household Expenditure Surveys 
Flours from cereal grains, roots, and tubers (continued)

\begin{tabular}{|c|c|c|}
\hline \multirow[t]{12}{*}{ Cassava, maize, millet (continued) } & Bag_-"minibag" or "maxibag" & Ghana \\
\hline & Basket & Burundi, Ghana \\
\hline & Basket—small, medium, large & Burundi \\
\hline & Bowl & Burundi, Ghana, Guinea \\
\hline & Bucket & Burundi \\
\hline & Calabash & Guinea \\
\hline & Can & Mozambique \\
\hline & Glass & Mozambique \\
\hline & Heap & Uganda \\
\hline & Margarine tin (Blueband) $-1 / 2,1$, and $2 \mathrm{~kg}$ & Uganda \\
\hline & Plate & Burundi \\
\hline & Sack-25, 50, or $100 \mathrm{~kg}$ (original content unspecified) & Burundi, Guinea \\
\hline \multicolumn{3}{|l|}{ Pulses } \\
\hline \multirow{12}{*}{$\begin{array}{l}\text { Dried beans and peas (bambara, } \\
\text { cowpeas, soybeans) }\end{array}$} & 1-kg Blueband tin & Uganda \\
\hline & $1 / 2$ liter plastic cup & Uganda \\
\hline & American tin, margarine tin & Ghana \\
\hline & Basket—small, medium, large & Burundi, Rwanda \\
\hline & Bowl & Burundi, Ghana, Rwanda \\
\hline & Bucket & Burundi \\
\hline & Can & Mozambique \\
\hline & Cooking pot—small, medium, large & Burundi \\
\hline & Cup & Burundi \\
\hline & Glass & Burundi, Mozambique \\
\hline & Glass-1/4-liter & Uganda \\
\hline & Goblet & Rwanda \\
\hline
\end{tabular}




\begin{tabular}{|c|c|c|}
\hline Food & Unit of measure & Country \\
\hline \multicolumn{3}{|l|}{ Pulses (continued) } \\
\hline \multirow[t]{9}{*}{ Dried beans and peas (continued) } & Heap & Burundi, Mozambique, Rwanda \\
\hline & Milk pot & Rwanda \\
\hline & Plate & Burundi \\
\hline & Plate & Rwanda \\
\hline & Plate_-“Nsima plate," “No. 10 plate," "relish plate” & Malawi \\
\hline & Sack-25, 50, or $100 \mathrm{~kg}$ (original content unspecified) & Burundi \\
\hline & Spoon & Rwanda \\
\hline & Tomato can & Rwanda \\
\hline & Various containers ("SIRVA," "metal," "plastic") & Rwanda \\
\hline \multicolumn{3}{|l|}{ Nuts } \\
\hline \multirow[t]{3}{*}{ Almonds } & Can & Mozambique \\
\hline & Glass & Mozambique \\
\hline & Heap & Mozambique \\
\hline \multirow[t]{13}{*}{ Groundnuts } & American tin, margarine tin & Ghana \\
\hline & Basket & Ghana \\
\hline & Bowl & Ghana, Rwanda \\
\hline & Can & Mozambique \\
\hline & Glass & Mozambique \\
\hline & Goblet & Rwanda \\
\hline & Heap & Mozambique, Rwanda \\
\hline & Plate & Rwanda \\
\hline & Plate_-“Nsima plate," “No. 10 plate," “relish plate” & Malawi \\
\hline & Rice bag-25 kg & Papua New Guinea \\
\hline & Small sack & Rwanda \\
\hline & Spoon & Rwanda \\
\hline & Tomato can & Rwanda \\
\hline \multirow[t]{2}{*}{ Groundnuts, shelled } & $1 / 2-\mathrm{kg}$ Blueband tin & Uganda \\
\hline & Glass & Uganda \\
\hline
\end{tabular}

106 Measuring Food Security Using Household Expenditure Surveys 


\begin{tabular}{|c|c|c|}
\hline Food & Unit of measure & Country \\
\hline \multicolumn{3}{|l|}{ Nuts (continued) } \\
\hline Groundnuts, unshelled & 1-kg Blueband tin & Uganda \\
\hline \multirow[t]{2}{*}{ Groundnuts, pounded } & Glass & Uganda \\
\hline & Heap & Uganda \\
\hline \multirow[t]{7}{*}{ Palmnuts } & American tin, margarine tin & Ghana \\
\hline & Basket & Ghana \\
\hline & Bowl & Ghana \\
\hline & Bunch & Guinea \\
\hline & Bundle & Ghana \\
\hline & Sack-50 or $100 \mathrm{~kg}$ (original content unspecified) & Guinea \\
\hline & Unities & Ghana \\
\hline \multirow[t]{2}{*}{ Coconuts } & Bundle & Ghana \\
\hline & Unities & $\begin{array}{l}\text { Ghana, Mozambique, Tanzania, India, Indonesia, } \\
\text { Papua New Guinea, Sri Lanka }\end{array}$ \\
\hline \multicolumn{3}{|l|}{ Seeds } \\
\hline \multirow[t]{3}{*}{ Sesame seeds } & $1 / 2-\mathrm{kg}$ Kimbo tin & Uganda \\
\hline & Glass & Mozambique \\
\hline & Glass-1/4 liter & Uganda \\
\hline \multicolumn{3}{|l|}{ Vegetables } \\
\hline \multicolumn{3}{|l|}{ Leafy greens } \\
\hline Bean leaves & Heap & Mozambique \\
\hline \multirow[t]{8}{*}{ Cassava leaves } & Basin & Rwanda \\
\hline & Basket—small, medium, large & Rwanda \\
\hline & Bowl & Rwanda \\
\hline & Heap & Mozambique, Rwanda \\
\hline & Plate & Rwanda \\
\hline & Sack-small, large & Rwanda \\
\hline & Unities & Guinea, Rwanda \\
\hline & Various containers ("SIRVA," “metal," "plastic") & Rwanda \\
\hline
\end{tabular}




\begin{tabular}{|c|c|c|}
\hline Food & Unit of measure & Country \\
\hline \multicolumn{3}{|l|}{ Vegetables (continued) } \\
\hline \multicolumn{3}{|l|}{ Leafy greens (continued) } \\
\hline Dodo & Bundle & Uganda \\
\hline Kale & Heap & Mozambique \\
\hline Potato leaves & Heap & Mozambique \\
\hline Pumpkin leaves & Heap & Mozambique \\
\hline \multirow[t]{5}{*}{ Spinach } & American tin & Ghana \\
\hline & Basket & Ghana \\
\hline & Bowl & Ghana \\
\hline & Bundle & Ghana \\
\hline & Margarine tin & Ghana \\
\hline \multirow[t]{3}{*}{ Cabbage } & Basket—small, medium, large & Burundi, Rwanda \\
\hline & Heap & Rwanda \\
\hline & Unities & $\begin{array}{l}\text { Burundi, Ghana, Guinea, Malawi, Mozambique, } \\
\text { Rwanda, Tanzania, Uganda }\end{array}$ \\
\hline \multirow[t]{9}{*}{ Carrots } & Basket & Ghana \\
\hline & Basket—small, medium, large & Burundi, Rwanda \\
\hline & Bowl & Ghana, Rwanda \\
\hline & Bundle & Burundi \\
\hline & Heap & Burundi, Mozambique, Rwanda, Uganda \\
\hline & Margarine tin & Ghana \\
\hline & Plate & Burundi, Rwanda \\
\hline & Unities & Ghana, Rwanda, Tanzania \\
\hline & Various containers ("SIRVA," "metal," "plastic") & Rwanda \\
\hline Corn, on cob ("green") & Unities & Malawi, Tanzania \\
\hline \multirow[t]{2}{*}{ Cucumbers } & Heap & Malawi, Mozambique \\
\hline & Unities & Guinea, Malawi, Mozambique \\
\hline
\end{tabular}

108 Measuring Food Security Using Household Expenditure Surveys 


\begin{tabular}{|c|c|c|}
\hline Food & Unit of measure & Country \\
\hline \multicolumn{3}{|l|}{ Vegetables (continued) } \\
\hline \multirow[t]{10}{*}{ Eggplant } & American tin & Ghana \\
\hline & Basket & Ghana \\
\hline & Basket—small, medium, large & Burundi, Rwanda \\
\hline & Bowl & Burundi, Ghana, Rwanda \\
\hline & Bucket & Burundi \\
\hline & Cooking pot—small, medium, large & Burundi \\
\hline & Heap & Burundi, Mozambique, Rwanda, Uganda \\
\hline & Margarine tin & Ghana \\
\hline & Unities & Burundi, Ghana, Guinea, Tanzania \\
\hline & Various containers ("SIRVA," "metal," "plastic") & Rwanda \\
\hline \multirow[t]{3}{*}{ Garlic } & Glass & Mozambique \\
\hline & Heap & Mozambique, Uganda \\
\hline & Unities & Mozambique \\
\hline \multirow[t]{3}{*}{ Gourds and squash } & Heap & Rwanda \\
\hline & Plate & Rwanda \\
\hline & Unities & Rwanda \\
\hline \multirow[t]{8}{*}{ Green beans } & Basin & Rwanda \\
\hline & Basket—small, medium, large & Rwanda \\
\hline & Bowl & Rwanda \\
\hline & Can & Mozambique \\
\hline & Glass & Mozambique \\
\hline & Heap & Mozambique, Rwanda, Uganda \\
\hline & Plate & Rwanda \\
\hline & Various containers ("SIRVA," "metal," "plastic") & Rwanda \\
\hline
\end{tabular}




\begin{tabular}{|c|c|c|}
\hline Food & Unit of measure & Country \\
\hline \multicolumn{3}{|c|}{ Vegetables (continued) } \\
\hline \multirow[t]{5}{*}{ Leeks } & Basket & Burundi \\
\hline & Bundle & Burundi \\
\hline & Heap & Burundi, Rwanda \\
\hline & Plate & Burundi, Rwanda \\
\hline & Unities & Burundi, Guinea, Rwanda, Tanzania \\
\hline \multirow[t]{2}{*}{ Lettuce } & Heap & Mozambique \\
\hline & Unities & Tanzania \\
\hline \multirow[t]{2}{*}{ Mushrooms } & Can & Mozambique \\
\hline & Heap & Mozambique \\
\hline \multirow[t]{7}{*}{ Okra } & American tin & Ghana \\
\hline & Basket & Ghana \\
\hline & Bowl & Ghana \\
\hline & Glass & Mozambique \\
\hline & Heap & Mozambique \\
\hline & Margarine tin & Ghana \\
\hline & Unities & Ghana, Guinea, Mozambique, Tanzania \\
\hline \multirow[t]{10}{*}{ Onions } & American tin & Ghana \\
\hline & Basin & Rwanda \\
\hline & Basket & Burundi, Ghana \\
\hline & Bowl & Burundi, Ghana, Rwanda \\
\hline & Bundle & Burundi, Ghana, Malawi \\
\hline & Cooking pot—small, medium, large & Burundi \\
\hline & Heap & Burundi, Mozambique, Rwanda, Uganda \\
\hline & Margarine tin & Ghana \\
\hline & Plate & Burundi, Rwanda \\
\hline & Unities & $\begin{array}{l}\text { Burundi, Ghana, Guinea, Malawi, Mozambique } \\
\text { Rwanda, Tanzania }\end{array}$ \\
\hline
\end{tabular}

110 Measuring Food Security Using Household Expenditure Surveys 


\begin{tabular}{|c|c|c|}
\hline Food & Unit of measure & Country \\
\hline \multicolumn{3}{|c|}{ Vegetables (continued) } \\
\hline \multirow[t]{3}{*}{ Peppers, green } & Bowl & Rwanda \\
\hline & Heap & Mozambique, Rwanda, Uganda \\
\hline & Unities & Mozambique, Rwanda, Tanzania \\
\hline \multirow[t]{7}{*}{ Peppers, hot } & Basin & Rwanda \\
\hline & Bowl & Rwanda \\
\hline & Heap & Rwanda \\
\hline & Spoon & Rwanda \\
\hline & Tomato can & Rwanda \\
\hline & Unities & Guinea, Rwanda \\
\hline & Various containers (“SIRVA", “metal”, "plastic”) & Rwanda \\
\hline Pumpkins & Unities & Mozambique \\
\hline \multirow[t]{13}{*}{ Tomatoes } & American tin & Ghana \\
\hline & Basin & Rwanda \\
\hline & Basket & Ghana \\
\hline & Basket—small, medium, large & Burundi, Rwanda \\
\hline & Bowl & Burundi, Ghana, Rwanda \\
\hline & Box & Ghana \\
\hline & Bundle & Malawi \\
\hline & Cooking pot- small, medium, large & Burundi \\
\hline & Heap & Burundi, Malawi, Mozambique, Rwanda, Uganda \\
\hline & Margarine tin & Ghana \\
\hline & Plate & Burundi, Rwanda \\
\hline & Unities & Burundi, Ghana, Malawi, Rwanda, Tanzania \\
\hline & Various containers ("SIRVA," "metal," "plastic") & Rwanda \\
\hline \multicolumn{3}{|l|}{ Fruits } \\
\hline Apples, pears & Unities & Tanzania \\
\hline
\end{tabular}




\begin{tabular}{|c|c|c|}
\hline Food & Unit of measure & Country \\
\hline \multicolumn{3}{|c|}{ Fruits (continued) } \\
\hline \multirow[t]{14}{*}{ Avocadoes } & Basin & Rwanda \\
\hline & Basket & Ghana \\
\hline & Basket—small, medium, large & Burundi, Rwanda \\
\hline & Bowl & Burundi \\
\hline & Bucket & Burundi \\
\hline & Cooking pot— small, medium, large & Burundi \\
\hline & Heap & Burundi, Rwanda \\
\hline & Pan & Rwanda \\
\hline & Plate & Burundi \\
\hline & Sack-25, 50, or $100 \mathrm{~kg}$ (original content unspecified) & Burundi \\
\hline & Sack—small, large & Rwanda \\
\hline & Unities & Burundi, Ghana, Guinea, Rwanda \\
\hline & Van & Rwanda \\
\hline & Various containers (“SIRVA," "metal," "plastic") & Rwanda \\
\hline \multirow[t]{8}{*}{ Bananas } & Basket—small, medium, large & Rwanda \\
\hline & Bowl & Rwanda \\
\hline & Bundle & Ghana \\
\hline & Cluster & Uganda \\
\hline & Heap & Mozambique, Rwanda \\
\hline & Plate & Rwanda \\
\hline & Rice bag-25 kg & Papua New Guinea \\
\hline & Unities & $\begin{array}{l}\text { Ghana, Guinea, Rwanda, Tanzania, India, } \\
\text { Pakistan, Papua New Guinea }\end{array}$ \\
\hline \multirow[t]{5}{*}{ Guavas } & Basin & Rwanda \\
\hline & Basket—small, medium, large & Rwanda \\
\hline & Bowl & Rwanda \\
\hline & Heap & Rwanda \\
\hline & Plate & Rwanda \\
\hline
\end{tabular}

112 Measuring Food Security Using Household Expenditure Surveys 


\begin{tabular}{|c|c|c|}
\hline Food & Unit of measure & Country \\
\hline \multicolumn{3}{|l|}{ Fruits (continued) } \\
\hline \multirow[t]{2}{*}{ Guavas (continued) } & Unities & Guinea, Rwanda \\
\hline & Various containers ("SIRVA," "metal," "plastic") & Rwanda \\
\hline \multirow[t]{13}{*}{ Mangoes } & American tin & Ghana \\
\hline & Basin & \\
\hline & Basket & Ghana \\
\hline & Basket—small, medium, large & Burundi \\
\hline & Bowl & Burundi, Ghana, Rwanda \\
\hline & Bucket & Burundi \\
\hline & Cooking pot—small, medium, large & Burundi \\
\hline & Heap & Burundi, Mozambique, Rwanda, Uganda \\
\hline & Plate & Burundi \\
\hline & Sack-25, 50, or $100 \mathrm{~kg}$ (original content unspecified) & Burundi \\
\hline & Sack—small, large & Rwanda \\
\hline & Unities & Burundi, Ghana, Guinea, Mozambique, Rwanda \\
\hline & Various containers ("SIRVA," "metal," "plastic") & Rwanda \\
\hline \multirow[t]{6}{*}{ Oranges, lemons, limes } & Basket & Ghana \\
\hline & Basket—small, medium, large & Burundi \\
\hline & Bowl & Ghana \\
\hline & Heap & Mozambique, Uganda \\
\hline & Minibag & Ghana \\
\hline & Unities & $\begin{array}{l}\text { Burundi, Ghana, Guinea, Mozambique, Tanzania, } \\
\text { India, Sri Lanka }\end{array}$ \\
\hline \multirow[t]{3}{*}{ Papayas } & Bowl & Ghana \\
\hline & Heap & Rwanda \\
\hline & Unities & Ghana, Guinea, Mozambique, Rwanda \\
\hline \multirow[t]{2}{*}{ Passion fruit } & Basin & Rwanda \\
\hline & Basket—small, medium, large & Rwanda \\
\hline
\end{tabular}




\begin{tabular}{|c|c|c|}
\hline Food & Unit of measure & Country \\
\hline \multicolumn{3}{|l|}{ Fruits (continued) } \\
\hline \multirow[t]{4}{*}{ Passion fruit (continued) } & Heap & Rwanda, Uganda \\
\hline & Plate & Rwanda \\
\hline & Unities & Rwanda \\
\hline & Various containers ("SIRVA," "metal," "plastic") & Rwanda \\
\hline \multirow[t]{4}{*}{ Pineapples } & American tin & Ghana \\
\hline & Basket—small, medium, large & Burundi \\
\hline & Sack-25, 50, or $100 \mathrm{~kg}$ (original content unspecified) & Guinea \\
\hline & Unities & $\begin{array}{l}\text { Burundi, Ghana, Guinea, Mozambique, Rwanda, } \\
\text { Tanzania, India }\end{array}$ \\
\hline \multirow[t]{2}{*}{ Watermelons } & Basket & Ghana \\
\hline & Unities & Ghana, Guinea, Mozambique \\
\hline \multicolumn{3}{|l|}{ Meats } \\
\hline \multirow[t]{3}{*}{ Beef } & Heap & Mozambique, Rwanda \\
\hline & Slice & Malawi \\
\hline & Unities & Ghana \\
\hline \multirow[t]{3}{*}{ Chicken and other birds } & Heap & Rwanda \\
\hline & Slice & Malawi \\
\hline & Unities & $\begin{array}{l}\text { Ghana, Guinea, Malawi, Mozambique, Rwanda, } \\
\text { Uganda, Papua New Guinea }\end{array}$ \\
\hline \multirow[t]{3}{*}{ Goat and lamb } & Heap & Mozambique \\
\hline & Slice & Malawi \\
\hline & Unities & $\begin{array}{l}\text { Ghana, Guinea, Mozambique, Rwanda, Papua } \\
\text { New Guinea }\end{array}$ \\
\hline \multirow[t]{3}{*}{ Pork } & Heap & Mozambique \\
\hline & Slice & Malawi \\
\hline & Unities & Ghana, Mozambique, Papua New Guinea \\
\hline Wild game and rabbit & Unities & Ghana, Mozambique \\
\hline
\end{tabular}

114 Measuring Food Security Using Household Expenditure Surveys 


\begin{tabular}{|c|c|c|}
\hline Food & Unit of measure & Country \\
\hline \multicolumn{3}{|l|}{ Fish and seafood } \\
\hline \multirow[t]{9}{*}{ Fish, fresh } & $1 / 2$-liter plastic cup & Uganda \\
\hline & $2-\mathrm{kg}$ tin & Uganda \\
\hline & Basket & Burundi \\
\hline & Bundle & Malawi \\
\hline & Cooking pot—small, medium, large & Burundi \\
\hline & Heap & Burundi, Malawi, Mozambique, Rwanda, Uganda \\
\hline & Plate-“No. 10 plate," "relish plate" & Malawi \\
\hline & $\mathrm{Sac}-25 \mathrm{~kg}$ & Burundi \\
\hline & Unities & $\begin{array}{l}\text { Burundi, Malawi, Mozambique, Rwanda, Uganda, } \\
\text { Papua New Guinea }\end{array}$ \\
\hline \multirow[t]{5}{*}{ Fish, dried or smoked } & Bowl & Rwanda \\
\hline & Can & Mozambique \\
\hline & Glass & Mozambique \\
\hline & Heap & Malawi, Mozambique \\
\hline & Unities & Malawi, Mozambique, Uganda \\
\hline \multirow[t]{2}{*}{ Crab } & Heap & Mozambique \\
\hline & Unities & Mozambique \\
\hline \multirow[t]{7}{*}{ Crustaceans } & American tin & Ghana \\
\hline & Basket & Ghana \\
\hline & Bowl & Ghana \\
\hline & Box & Ghana \\
\hline & Finger & Ghana \\
\hline & Margarine tin & Ghana \\
\hline & Unities & Ghana \\
\hline Oysters & Glass & Mozambique \\
\hline Sardines & Can & Mozambique \\
\hline
\end{tabular}




\begin{tabular}{|c|c|c|}
\hline Food & Unit of measure & Country \\
\hline \multicolumn{3}{|l|}{ Fish and seafood (continued) } \\
\hline \multirow[t]{3}{*}{ Shrimp } & Can & Mozambique \\
\hline & Glass & Mozambique \\
\hline & Heap & Mozambique \\
\hline \multicolumn{3}{|l|}{ Milk and dairy products } \\
\hline \multirow[t]{14}{*}{ Milk, fresh, sour, or condensed } & $1 / 2$-liter plastic cup & Uganda \\
\hline & Beer bottle & Ghana \\
\hline & Bottle & Uganda \\
\hline & Bottle-30, $33,65,72$, or 75 centiliter or 1 liter & Burundi \\
\hline & Bottle-Fanta, Primus, whiskey, or martini & Rwanda \\
\hline & Bowl & Ghana \\
\hline & Can & Mozambique \\
\hline & Can-5 liter & Rwanda \\
\hline & Can- $5,20,30$, or 50 liter & Burundi \\
\hline & Cup & Burundi \\
\hline & Glass & Burundi \\
\hline & Goblet & Rwanda \\
\hline & Milk pot & Rwanda \\
\hline & Various containers ("SIRVA," "metal," "plastic") & Rwanda \\
\hline Milk, dried & Box-small, medium & Burundi \\
\hline \multirow[t]{5}{*}{ Eggs } & Basket & Ghana \\
\hline & Bundle & Malawi \\
\hline & Heap & Malawi, Rwanda \\
\hline & Margarine tin & Ghana \\
\hline & Unities & $\begin{array}{l}\text { Ethiopia, Ghana, Guinea, Malawi, Mozambique, } \\
\text { Rwanda, Tanzania, Bangladesh, India, Indonesia, } \\
\text { Pakistan, Papua New Guinea, Sri Lanka }\end{array}$ \\
\hline
\end{tabular}

116 Measuring Food Security Using Household Expenditure Surveys 


\begin{tabular}{|c|c|c|}
\hline Food & Unit of measure & Country \\
\hline \multicolumn{3}{|l|}{ Beverages } \\
\hline \multirow[t]{15}{*}{ Beer } & $2-\mathrm{kg}$ tin & Uganda \\
\hline & Beer bottle & Uganda \\
\hline & Bottle & Mozambique \\
\hline & Bowl & Rwanda \\
\hline & Calabash & Rwanda \\
\hline & Can & Mozambique \\
\hline & Can-1 or 5 liter & Rwanda \\
\hline & Goblet & Rwanda \\
\hline & Jerrican & Rwanda \\
\hline & Jug & Rwanda \\
\hline & Martini bottle & Rwanda \\
\hline & Primus bottle & Rwanda \\
\hline & Soda bottle & Rwanda \\
\hline & Various containers ("SIRVA," "metal," "plastic") & Rwanda \\
\hline & Whiskey bottle & Rwanda \\
\hline \multirow[t]{4}{*}{ Coffee } & Can & Mozambique \\
\hline & Cup & Bangladesh, India \\
\hline & Packet & Uganda \\
\hline & Sack—50 or $100 \mathrm{~kg}$ & Guinea \\
\hline \multirow[t]{7}{*}{ Fruit juice } & Bottle & Mozambique \\
\hline & Bottle-1/2 liter & Uganda \\
\hline & Bottle-30 centiliter & Burundi \\
\hline & Calabash & Rwanda \\
\hline & Can-1 or 5 liter & Rwanda \\
\hline & Glass & India, Pakistan \\
\hline & Jerrican & Rwanda \\
\hline
\end{tabular}




\begin{tabular}{|c|c|c|}
\hline Food & Unit of measure & Country \\
\hline \multicolumn{3}{|l|}{ Beverages (continued) } \\
\hline \multirow[t]{6}{*}{ Fruit juice (continued) } & Martini bottle & Rwanda \\
\hline & Plastic cup-1/2 liter & Uganda \\
\hline & Primus bottle & Rwanda \\
\hline & Soda bottle & Rwanda, Uganda \\
\hline & Various containers ("SIRVA," "metal," "plastic") & Rwanda \\
\hline & Whiskey bottle & Rwanda \\
\hline \multirow[t]{2}{*}{ Soft drinks (Coca Cola, Fanta, etc.) } & Bottle & Mozambique, Pakistan \\
\hline & Can & Mozambique \\
\hline \multirow[t]{7}{*}{ Tea } & Bottle cover & Mozambique \\
\hline & Can & Mozambique \\
\hline & Cup & Bangladesh, India \\
\hline & Glass & Mozambique \\
\hline & Packet -50 or 100 gram & Uganda \\
\hline & Plastic container-1, 2, or 3 liter & Uganda \\
\hline & Teaspoon & Mozambique \\
\hline \multicolumn{3}{|l|}{ Beverages (continued) } \\
\hline \multirow[t]{6}{*}{ Traditional drinks } & Bottle & Mozambique \\
\hline & Bottle- $-33,65,72$, or 75 centiliter and 1 liter & Burundi \\
\hline & Bucket & Burundi \\
\hline & Cooking pot—small, medium, large & Burundi \\
\hline & Cup & Burundi \\
\hline & Tin-5, 20, 30, 50, or 100 liter & Burundi \\
\hline \multicolumn{3}{|l|}{ Miscellaneous } \\
\hline \multirow[t]{3}{*}{ Curry powder } & Bottle cover & Mozambique \\
\hline & Heap & Mozambique \\
\hline & Teaspoon & Mozambique \\
\hline
\end{tabular}

118 Measuring Food Security Using Household Expenditure Surveys 


\begin{tabular}{|c|c|c|}
\hline Food & Unit of measure & Country \\
\hline \multicolumn{3}{|c|}{ Miscellaneous (continued) } \\
\hline \multirow[t]{3}{*}{ Fresh hot pepper } & Can & Mozambique \\
\hline & Heap & Mozambique \\
\hline & Teaspoon & Mozambique \\
\hline Ground black pepper & Teaspoon & Mozambique \\
\hline \multirow[t]{9}{*}{ Honey } & Bottle-33, 65,72 , or 75 centiliter or 1 liter & Burundi \\
\hline & Cup & Burundi \\
\hline & Glass & Burundi \\
\hline & Goblet & Rwanda \\
\hline & Primus bottle & Rwanda \\
\hline & Soda bottle & Rwanda \\
\hline & Spoon & Burundi, Rwanda \\
\hline & Tin-5 liter & Burundi \\
\hline & Whiskey bottle & Rwanda \\
\hline \multirow[t]{9}{*}{ Salt } & Bottle cover & Mozambique \\
\hline & Box-medium & Burundi \\
\hline & Can & Mozambique \\
\hline & Cup & Burundi, Uganda \\
\hline & Glass & Burundi, Mozambique, Uganda \\
\hline & Heap & Burundi \\
\hline & Packet—500 grams & Uganda \\
\hline & Spoon & Burundi \\
\hline & Teaspoon & Mozambique \\
\hline \multirow[t]{4}{*}{ Sugar } & Bottle cover & Mozambique \\
\hline & Can & Mozambique \\
\hline & Glass & Mozambique, Uganda \\
\hline & Spoon & Burundi \\
\hline
\end{tabular}




\section{Appendix 3}

\section{Cup Weights of Commonly Consumed Foods}

\begin{tabular}{|c|c|c|c|}
\hline \multirow[b]{2}{*}{ Food } & \multicolumn{3}{|c|}{ Cup weight (grams) } \\
\hline & Malaysia & Senegal & $\begin{array}{l}\text { United } \\
\text { States }\end{array}$ \\
\hline \multicolumn{4}{|l|}{ Grains, roots, and tubers } \\
\hline Barley, grain & 197 & & 184 \\
\hline Barley, flour & & & 148 \\
\hline Cassava, flour & 118 & & \\
\hline Couscous (wheat) & & & 173 \\
\hline Fonio & 133 & & \\
\hline Maize, grain & 210 & 220 & 166 \\
\hline Maize, flour & & & 126 \\
\hline Maize, meal & & & 138 \\
\hline Maize, broken grains & 210 & & \\
\hline Millet, grain & 133 & 191 & 200 \\
\hline Millet, flour & & 118 & \\
\hline Rice & 190 & 220 & \\
\hline Rice, flour & 119 & & 158 \\
\hline Rice, glutinous & 179 & & 185 \\
\hline Rice, long-grain & 173 & & 185 \\
\hline Rice, medium-grain & 175 & & 195 \\
\hline Rice, short-grain & 176 & & 200 \\
\hline Sorghum & & & 192 \\
\hline Wheat, grain & 188 & & 192 \\
\hline Wheat, flour & 133 & & 125 \\
\hline
\end{tabular}

\begin{tabular}{|c|c|c|c|}
\hline \multirow[b]{2}{*}{ Food } & \multicolumn{3}{|c|}{ Cup weight (grams) } \\
\hline & Malaysia & Senegal & $\begin{array}{l}\text { United } \\
\text { States }\end{array}$ \\
\hline \multicolumn{4}{|l|}{ Pulses and legumes } \\
\hline Beans, black & & & 194 \\
\hline Beans, broad & & & 150 \\
\hline Beans, kidney & & & 184 \\
\hline Beans, lentils & & & 192 \\
\hline Beans, mung & & & 207 \\
\hline Beans, mung (gram, black) & 168 & & \\
\hline Beans, mung (gram, green) & 187 & & \\
\hline Beans, pinto & & & 193 \\
\hline Beans, red & 183 & & \\
\hline Beans, soya & 167 & & 186 \\
\hline Chickpeas & 176 & & 200 \\
\hline Cowpeas & & & 167 \\
\hline Nuts, almonds & & & 95 \\
\hline Nuts, groundnuts (shelled) & 146 & & 146 \\
\hline Nuts, walnuts & & & 120 \\
\hline Peas & 149 & & \\
\hline Pigeon peas & 176 & & 205 \\
\hline Sesame seeds & & & 144 \\
\hline $\begin{array}{l}\text { Sunflower seeds } \\
\text { (dry, roasted with salt) }\end{array}$ & & & 148 \\
\hline
\end{tabular}


Cup weight (grams)

Food

Malaysia Senegal States

\section{Sugar and spices}

\begin{tabular}{|c|c|}
\hline Black pepper & 101 \\
\hline Cardamom & 92 \\
\hline Chili powder & 119 \\
\hline Cinnamon & 107 \\
\hline Cloves, ground & 104 \\
\hline Coriander leaf, dried & 28 \\
\hline Cumin, seed & 95 \\
\hline Curry powder & 100 \\
\hline Fennel, seed & 92 \\
\hline Ginger, ground & 85 \\
\hline Mint, fresh (peppermint) & 51 \\
\hline Mustard seed, yellow & 177 \\
\hline Nutmeg & 111 \\
\hline Parsley & 21 \\
\hline Salt & 292 \\
\hline Sugar, brown & 220 \\
\hline Sugar, white & 200 \\
\hline Tumeric & 107 \\
\hline
\end{tabular}

Sources: Malaysia—Siong et al. (1997), Senegal—Dop et al. (2003), United StatesUSDA (2003, 2005).

Note: One cup is assumed to contain 237 milliliters. 


\section{Appendix 4}

Specific Gravities of Commonly Consumed Beverages and Other Liquid Foods

\begin{tabular}{l|c|c|c}
\multicolumn{2}{l}{ Food } & \multicolumn{3}{c}{ Grams per milliliter } \\
\cline { 2 - 4 } Milk and milk products & $\begin{array}{c}\text { United } \\
\text { States }\end{array}$ & $\begin{array}{c}\text { Australia and } \\
\text { New Zealand }\end{array}$ \\
Buttermilk & & 1.03 & 1.04 \\
\hline Cream & 1.00 & 1.02 & 1.01 \\
\hline Curd & & 0.95 & \\
\hline $\begin{array}{l}\text { Milk, condensed, } \\
\text { sweetened }\end{array}$ & 1.40 & 1.29 & 1.29 \\
\hline Milk, cow, whole & 1.02 & 1.03 & 1.03 \\
\hline Milk, curdled & & 0.96 & \\
\hline $\begin{array}{l}\text { Milk, evaporated, } \\
\text { unsweetened }\end{array}$ & 1.04 & 1.08 & 1.07 \\
\hline Yogurt & 1.03 & 1.03 & 1.04 \\
\hline Oils and oil products & & & \\
\hline Mayonnaise & & 0.93 & 0.99 \\
\hline Oil, coconut & & & 0.92 \\
\hline Oil, groundnut & 0.87 & 0.91 & 0.91 \\
\hline Oil, palm & 0.80 & & 0.92 \\
\hline Oil, sesame seed & 0.87 & 0.92 & 0.92 \\
\hline Oil, soybean & 0.87 & 0.92 & 0.92 \\
\hline Oil, vegetable & & 0.92 & 0.92 \\
\hline
\end{tabular}

\begin{tabular}{|c|c|c|c|}
\hline \multirow[b]{2}{*}{ Food } & \multicolumn{3}{|c|}{ Grams per milliliter } \\
\hline & Malaysia & $\begin{array}{l}\text { United } \\
\text { States }\end{array}$ & $\begin{array}{l}\text { Australia and } \\
\text { New Zealand }\end{array}$ \\
\hline \multicolumn{4}{|l|}{ Nonalcoholic beverages } \\
\hline Coffee & & 1.01 & 1.01 \\
\hline Cordials or concentrates & & & 1.13 \\
\hline $\begin{array}{l}\text { Green (young) coconut } \\
\text { water }\end{array}$ & 1.00 & & \\
\hline Juice, apple & & 1.05 & 1.05 \\
\hline Juice, grape & & 1.07 & 1.05 \\
\hline Juice, grapefruit & & 1.04 & 1.05 \\
\hline Juice, lemon & & 1.03 & 1.05 \\
\hline Juice, mango & & & 1.05 \\
\hline Juice, orange & & 1.05 & 1.05 \\
\hline Juice, pineapple & & 1.05 & 1.05 \\
\hline Juice, tangerine & & 1.05 & \\
\hline Soft drinks & & 1.04 & 1.04 \\
\hline Tea & & 1.00 & 1.00 \\
\hline Water, carbonated & & & 1.04 \\
\hline Water, mineral & & & 1.00 \\
\hline
\end{tabular}


Grams per milliliter

\begin{tabular}{l|r|r|r}
\cline { 3 - 4 } \multicolumn{2}{c}{ Food } & \multicolumn{3}{c}{ Grams per milliliter } \\
\cline { 3 - 4 } Alcoholic beverages & & $\begin{array}{c}\text { United } \\
\text { States }\end{array}$ & $\begin{array}{c}\text { Australia and } \\
\text { New Zealand }\end{array}$ \\
Beer & & 1.04 & 1.01 \\
\hline Brandy & & 0.99 & 0.96 \\
\hline Sherry & & 1.04 & 1.04 \\
\hline Vermouth & & 1.04 & \\
\hline Whiskey & & 0.99 & 0.95 \\
\hline Wine & & 1.04 & 1.01
\end{tabular}

Miscellaneous

\begin{tabular}{l|c|c|c} 
Fish, oyster sauce & 1.20 & & 1.22 \\
\hline Honey & 1.40 & 1.43 & 1.43 \\
\hline Ice cream (vanilla) & & 0.56 & 0.56 \\
\hline Molasses & & 1.33 & 1.39 \\
\hline Soya sauce & & 1.07 & 1.05 \\
\hline Soya sauce, white & 1.13 & & 1.05 \\
\hline Soya sauce, black & 1.33 & & \\
\hline Syrup & 1.20 & 1.27 & 1.20 \\
\hline Tomato sauce, ketchup & 1.20 & 1.03 & 1.04 \\
\hline Vinegar & 0.80 & 1.07 & 1.01
\end{tabular}

Sources: Australia and New Zealand_FSANZ (2004), Malaysia-Siong et al. (1997), United States-USDA $(2003,2005)$. 


\section{Appendix 5}

Divergence of Market Prices from Unit Values for 12 Foods, Papua New Guinea Household Survey 1995-96: Comparison of Gibson and Rozelle and AFINS Project Results

\begin{tabular}{|c|c|c|c|c|c|}
\hline \multirow[b]{2}{*}{ Food } & \multirow[b]{2}{*}{ Mean market price } & \multicolumn{2}{|c|}{ Gibson and Rozelle } & \multicolumn{2}{|c|}{ AFINS project } \\
\hline & & Mean unit value & $\begin{array}{c}\text { Percentage } \\
\text { difference from } \\
\text { mean market price }\end{array}$ & Mean unit value ${ }^{a}$ & $\begin{array}{c}\text { Percentage } \\
\text { difference from } \\
\text { mean market price }\end{array}$ \\
\hline Sweet potatoes & 43.9 & 59.0 & -25.6 & 44.3 & -0.9 \\
\hline Bananas & 54.2 & 75.9 & -28.6 & 63.4 & -14.5 \\
\hline Rice & 114.7 & 107.3 & 6.9 & 110.8 & 3.5 \\
\hline Flour & 143.6 & 114.9 & 25.0 & 128.5 & 11.8 \\
\hline Biscuits & 444.4 & 450.0 & -1.2 & 482.1 & -7.8 \\
\hline Canned fish & 432.7 & 437.0 & -1.0 & 460.0 & -5.9 \\
\hline Soft drinks & 272.8 & 263.3 & 3.6 & 279.1 & -2.3 \\
\hline Beer & 558.3 & 507.0 & 10.1 & 517.3 & 7.9 \\
\hline $\begin{array}{l}\text { Total absolute value difference from } \\
\text { mean market price }\end{array}$ & & & 102.0 & & 54.6 \\
\hline
\end{tabular}

Note: Mean unit values are in Toea per $\mathrm{kg}$ calculated from cluster-level means.

${ }^{a}$ The unit values used are those calculated after manual unit value cleaning by food and region, dropping households with large numbers of outlier metric quantities and predicting the remaining missing and outlying quantities following the procedures outlined in this manual. After the quantity predictions, it is possible that some reported quantities are now more in line with reported expenditures but also that some are not. Thus it is necessary to re-check for large outliers before calculating the mean unit values, as was done here. 


\section{Appendix 6}

\section{Edible Percentages of Commonly Consumed Foods}

\begin{tabular}{|c|c|c|c|c|}
\hline Food & Malaysia & United States & Other areas & (country or region) \\
\hline \multicolumn{5}{|l|}{ Roots and tubers } \\
\hline Cassava & & & 74 & Africa \\
\hline Cassava & & & 82 & Senegal \\
\hline Cassava & & & 73 & East Asia \\
\hline Plantain bananas & & & 69 & East Asia \\
\hline Plantain bananas & & & 66 & Africa \\
\hline Potatoes (English) & 90 & & 87 & East Asia \\
\hline Potatoes (English) & & & 86 & Africa \\
\hline Potatoes (English) & & & 83 & Senegal \\
\hline Sweet potatoes & 95 & & 79 & Africa \\
\hline Sweet potatoes & & & 87 & East Asia \\
\hline Sweet potatoes & & & 81 & Senegal \\
\hline Taro & 86 & & 84 & East Asia \\
\hline Taro & & & 84 & Africa \\
\hline Yams & & & 84 & East Asia \\
\hline \multicolumn{5}{|l|}{ Nuts, unshelled } \\
\hline Cashew nuts & 69 & & & \\
\hline Coconuts & 70 & 52 & 65 & Africa \\
\hline Groundnuts, peanuts & & & 73 & India \\
\hline Groundnuts, peanuts & & & 65 & East Asia \\
\hline Groundnuts, peanuts & & & 65 & Tropical areas (Platt) \\
\hline Groundnuts, peanuts & & & 74 & Senegal \\
\hline Walnuts & 43 & 45 & 45 & East Asia \\
\hline
\end{tabular}




\begin{tabular}{|c|c|c|c|c|}
\hline Food & Malaysia & United States & Other areas & (country or region) \\
\hline \multicolumn{5}{|l|}{ Vegetables } \\
\hline Asparagus & 67 & 53 & 66 & East Asia \\
\hline Bamboo shoots & & 29 & 33 & East Asia \\
\hline Beans, spring & 91 & & & \\
\hline Beans, green, French-cut, string & 90 & 94 & 95 & East Asia \\
\hline Beans, green, French-cut, string & & & 92 & East Africa \\
\hline Bean, sprouts & 100 & 100 & & \\
\hline Beets & 82 & 67 & 84 & East Asia \\
\hline Broccoli & 100 & 61 & 61 & East Asia \\
\hline Cabbage & 87 & 80 & 85 & East Asia \\
\hline Cabbage & & & 85 & Senegal \\
\hline Cabbage, Chinese & 100 & 88 & 86 & East Asia \\
\hline Carrots & 89 & 89 & 83 & East Asia \\
\hline Carrots & & & 87 & Senegal \\
\hline Cauliflower & & 39 & 60 & East Asia \\
\hline Cauliflower & & & 56 & Africa \\
\hline Celery & 69 & 89 & 75 & East Asia \\
\hline Cucumbers & 100 & 97 & 80 & East Asia \\
\hline Eggplant & 91 & 81 & 91 & East Asia \\
\hline Eggplant & & & 78 & Africa \\
\hline Eggplant & & & 91 & Kenya \\
\hline Fern shoots & 43 & & 43 & East Asia \\
\hline Garlic & 92 & 87 & 90 & East Asia \\
\hline Gourds, bottle & 86 & & 85 & East Asia \\
\hline Gourds, snake or ribbed & 97 & & & \\
\hline Gourds, wax & & 71 & 72 & East Asia \\
\hline Gourds, bitter & 90 & 83 & 80 & East Asia \\
\hline
\end{tabular}

126 Measuring Food Security Using Household Expenditure Surveys 


\begin{tabular}{|c|c|c|c|c|}
\hline Food & Malaysia & United States & Other areas & (country or region) \\
\hline \multicolumn{5}{|l|}{ Vegetables (continued) } \\
\hline Green peas, unshelled & & & 45 & East Asia \\
\hline Kale & & 61 & 74 & East Asia \\
\hline Kale & & & 68 & Africa \\
\hline Leaves, cassava & & & 87 & East Asia \\
\hline Leaves, cassava & & & 80 & East Africa \\
\hline Leaves, coriander & 80 & 85 & & \\
\hline Leaves, mustard & 85 & 93 & & \\
\hline Leaves, sweet potato & & 94 & 82 & East Asia \\
\hline Leaves, sweet potato & & & 80 & East Africa \\
\hline Leeks & 50 & 44 & 72 & East Asia \\
\hline Lettuce & 74 & 74 & 74 & East Asia \\
\hline Maize, on cob, with husk & & 36 & 37 & East Asia \\
\hline Maize, on cob, without husk & & & 70 & Africa \\
\hline Maize, on cob, without husk & & & 85 & Ghana \\
\hline Maize, on cob, without husk & & & 74 & East Asia \\
\hline Mushrooms & 100 & 97 & 91 & East Asia \\
\hline Mustard greens & 90 & 93 & 90 & East Asia \\
\hline Okra & 89 & 86 & 90 & East Asia \\
\hline Okra & & & 87 & Senegal \\
\hline Onions & 95 & 90 & 94 & East Asia \\
\hline Peppers, green, sweet & 88 & 82 & 87 & East Asia \\
\hline Peppers, chili, hot & 95 & 73 & 87 & East Asia \\
\hline Pumpkins & 74 & 70 & 83 & East Asia \\
\hline Radishes & & 90 & 73 & East Asia \\
\hline Radishes, Chinese & 91 & 79 & 87 & East Asia \\
\hline Spinach & 78 & 72 & 76 & East Asia \\
\hline
\end{tabular}




\section{Food}

Malaysia

United States

Other areas (country or region)

Vegetables (continued)

\begin{tabular}{l|l|l|ll} 
Squash, winter & & 76 & 74 & East Asia \\
\hline Squash, zucchini & & 95 & 87 & East Asia \\
\hline Swiss chard & & 92 & 90 & East Asia \\
\hline Tapioca shoots & 74 & 88 & & \\
\hline Tomatoes & 88 & 91 & 94 & East Asia \\
\hline Turnips & 84 & 81 & 79 & East Asia \\
\hline Watercress & 80 & 92 & 84 & East Asia
\end{tabular}

Fruits

\begin{tabular}{|c|c|c|c|c|}
\hline Apples & 90 & 92 & 90 & Kenya \\
\hline Avocadoes & 70 & 74 & 70 & East Asia \\
\hline Bananas & 69 & 64 & 68 & Africa \\
\hline Bananas & & & 65 & Senegal \\
\hline Blackberries & & 96 & 99 & Tropical areas (Platt) \\
\hline Cantaloupes & & 51 & 72 & East Asia \\
\hline Cashew apples, pulp & 70 & & & \\
\hline Cashew fruit & & & 87 & East Asia \\
\hline Cherries & & 90 & 82 & East Asia \\
\hline Custard apples & 56 & 58 & & \\
\hline Dates & 88 & 90 & 87 & Tropical areas (Platt) \\
\hline Durians & 22 & 32 & 25 & East Asia \\
\hline Gooseberries & & 100 & & \\
\hline Grapefruit & 64 & 50 & 51 & Africa \\
\hline Grapes & 95 & 96 & 94 & Africa \\
\hline Guavas & 98 & 80 & 81 & Africa \\
\hline Jack fruit & 28 & 28 & 30 & India \\
\hline Lanzon & 41 & & & \\
\hline
\end{tabular}

128 Measuring Food Security Using Household Expenditure Surveys 
Fruits (continued)

\begin{tabular}{|c|c|c|c|c|}
\hline Leeches & 65 & 60 & & \\
\hline Lemons & 35 & 53 & 64 & Africa \\
\hline Limes & 63 & 84 & 77 & East Asia \\
\hline Longan & & 53 & 50 & East Asia \\
\hline Lychee & 65 & 54 & 68 & India \\
\hline Mangoes & 75 & 69 & 64 & Africa \\
\hline Mangoes & & & 71 & Senegal \\
\hline Mangosteens & 24 & & 29 & East Asia \\
\hline Oranges & 73 & 73 & 72 & Africa \\
\hline Passion fruit & & 52 & 61 & East Asia \\
\hline Papayas & 70 & 67 & 74 & Africa \\
\hline Peaches & 94 & 87 & 90 & Africa \\
\hline Pears & 95 & 92 & 82 & Tropical areas (Platt) \\
\hline Pineapples & 59 & 52 & 67 & Africa \\
\hline Plums & 94 & 94 & 91 & Tropical areas (Platt) \\
\hline Pomegranates & & 56 & 56 & Tropical areas (Platt) \\
\hline Pomelos & 53 & 56 & & \\
\hline Raisins & 100 & 100 & 100 & India \\
\hline Sepadila & 82 & & 77 & Tropical areas (Platt) \\
\hline Snake fruit & 66 & & & \\
\hline Star fruit (carmabola) & 90 & 95 & 85 & East Asia \\
\hline Strawberries & 95 & 85 & 96 & India \\
\hline Tamarinds & 70 & 66 & & \\
\hline Tangerines & 73 & 72 & 72 & Africa \\
\hline Water apples & 96 & & 90 & East Asia \\
\hline Watermelons & 52 & 52 & 55 & Africa \\
\hline
\end{tabular}




\begin{tabular}{|c|c|c|c|c|}
\hline Food & Malaysia & United States & Other areas & (country or region) \\
\hline \multicolumn{5}{|l|}{ Meats } \\
\hline Beef & & 89 & 80 & East Asia \\
\hline Beef & & & 81 & Ghana \\
\hline Beef & & & 80 & East Africa \\
\hline Beef & & & 80 & Tropical areas (Platt) \\
\hline Beef & & & 78 & Senegal \\
\hline Chicken & 70 & 69 & 67 & East Africa \\
\hline Chicken & & & 58 & East Asia \\
\hline Chicken & & & 67 & Tropical areas (Platt) \\
\hline Duck & 39 & 38 & & \\
\hline Goat & & & 74 & East Africa \\
\hline Goat & & & 86 & East Asia \\
\hline Goat & & & 74 & Tropical areas (Platt) \\
\hline Goose & & 81 & 66 & East Asia \\
\hline Guinea fowl & & 83 & & \\
\hline Lamb & & 78 & 78 & East Asia \\
\hline Offals & & & 100 & East Asia \\
\hline Offals (liver) & 100 & 100 & & \\
\hline Pigeon & & 65 & 47 & East Asia \\
\hline Pork & 93 & 89 & 88 & East Asia \\
\hline Sausages & 100 & 100 & & \\
\hline Turkey & & 79 & 61 & East Asia \\
\hline Wild game_-general & & & 80 & Ghana \\
\hline Wild game, rabbit & 34 & & 82 & Tropical areas (Platt) \\
\hline Wild game, snail & & & 47 & Tropical areas (Platt) \\
\hline Wild game, squirrel & & & 60 & East Asia \\
\hline
\end{tabular}

130 Measuring Food Security Using Household Expenditure Surveys 


\begin{tabular}{|c|c|c|c|c|}
\hline Food & Malaysia & United States & Other areas & (country or region) \\
\hline \multicolumn{5}{|l|}{ Seafood } \\
\hline \multicolumn{5}{|l|}{ Fish } \\
\hline Fish, fresh-general & & & 52 & Africa \\
\hline Anchovies & 43 & & 73 & East Asia \\
\hline Bream, African & 40 & & 52 & East Asia \\
\hline Bream, threadfin, Japanese & 44 & & & \\
\hline Carp & 48 & & 57 & East Asia \\
\hline Carp & & & 52 & Africa \\
\hline Carp, Javanese & 55 & & & \\
\hline Catfish & 54 & & 54 & East Asia \\
\hline Catfish & & & 63 & Africa \\
\hline Catfish eel & 59 & & & \\
\hline Cod & 42 & & 52 & East Asia \\
\hline Cuttlefish & 91 & & 79 & East Asia \\
\hline Dart, ladyfish, or moonfish & 53 & & 48 & East Asia \\
\hline Gouramy & 48 & & & \\
\hline Grouper & 44 & & 46 & East Asia \\
\hline Herring & 55 & & 65 & East Asia \\
\hline Herring, round & 49 & & & \\
\hline Herring, wolf & 74 & & & \\
\hline Jewfish (croaker) & 38 & & 55 & East Asia \\
\hline Jewfish (croaker) & & & 56 & Africa \\
\hline Mackerel & 62 & & 59 & East Asia \\
\hline Mackerel & & & 63 & Africa \\
\hline Mullet & 45 & & 52 & East Asia \\
\hline Mullet & & & 61 & Africa \\
\hline
\end{tabular}




\begin{tabular}{|c|c|c|c|c|}
\hline Food & Malaysia & United States & Other areas & (country or region) \\
\hline \multicolumn{5}{|l|}{ Seafood (continued) } \\
\hline Perch & & & 52 & East Asia \\
\hline Perch & & & 56 & Africa \\
\hline Pomfret & 57 & & 64 & East Asia \\
\hline Ponyfish & 50 & & & \\
\hline Salmon & & & 63 & East Asia \\
\hline Sardines & 100 & & 58 & East Asia \\
\hline Scad & 47 & & 53 & East Asia \\
\hline Shad & 49 & & 48 & East Asia \\
\hline Shad & & & 70 & Africa \\
\hline Shark, dog & 34 & & 55 & East Asia \\
\hline Snakehead & 46 & & 57 & East Asia \\
\hline Snapper, red & 51 & & 55 & East Asia \\
\hline Stingray & 40 & & 41 & East Asia \\
\hline Threadfin & 41 & & 40 & East Asia \\
\hline Tilapia & & & 38 & East Asia \\
\hline Tilapia & & & 51 & East Asia \\
\hline Trevally, yellow banded & 48 & & & \\
\hline Tuna & 54 & & 71 & East Asia \\
\hline Yellowtail & 44 & & 61 & East Asia \\
\hline \multicolumn{5}{|l|}{ Other seafood } \\
\hline Clams & 48 & & 30 & East Asia \\
\hline Cockles & 30 & & 12 & East Asia \\
\hline Crab & 53 & & 34 & East Asia \\
\hline Crustaceans, etc.—general & & & 37 & Tropical areas (Platt) \\
\hline Lobster & 60 & & 47 & East Asia \\
\hline
\end{tabular}

132 Measuring Food Security Using Household Expenditure Surveys 
Seafood (continued)

\begin{tabular}{|c|c|c|c|}
\hline Molluscs, etc.-general & & 25 & Tropical areas (Platt) \\
\hline Mussels & 20 & 36 & East Asia \\
\hline Oysters & & 18 & East Asia \\
\hline Prawns (shrimp) & 66 & 52 & East Asia \\
\hline Prawns (shrimp) & & 45 & Kenya \\
\hline Snails & & 54 & East Asia \\
\hline Snails & & 65 & Africa \\
\hline Squid & & 93 & East Asia \\
\hline
\end{tabular}

Eggs

100

\begin{tabular}{l|c|c|cl} 
Chicken eggs & 87 & 88 & 89 & East Asia \\
\hline Chicken eggs & & & 89 & Senegal \\
\hline Duck eggs & 89 & 100 & 88 & East Asia \\
\hline Quail eggs & 85 & 92 & 89 & East Asia
\end{tabular}

Sources: Africa—Leung (1968); East Africa—West, Pepping, and Temalilwa (1988); East Asia—Leung, Butrum, and Chang (1972); GhanaEyeson and Ankrah (1975); India_Gopalan, Sastri, and Balasubramanian (1976); Kenya_Sehmi (1993); Malaysia—Siong et al. (1997); Senegal—Ndaiye (1993); tropical areas—Platt (1962); United States_USDA (2003, 2005).

Note: Some values are averages of varieties listed in the tables. 


\section{Appendix 7}

\section{STATA 8.0 Program for Calculating the Number of Household Adult Equivalents and Household Energy Requirements}

T

he data file used (termed "hhroster.dta") should contain the age and sex of each individual included in the survey who is defined to be a household member. Normally these data are in the file containing the data collected in the "household roster" module of a survey. Note: The variable "agey" is age in years. The energy requirements used in this program are from Table 7. More up-to-date requirements can be found in Appendix 8.

\section{\#delim;}

clear;

use hhroster.dta;

Part 1: Calculate number of household adult

cor missing and unrealistic

values;

rename age agey:

tab agey, missing

* None;

sex variable for missing and unrealistic

values;

tab sex,missing;

* None

$\star \star \star$ Create age categories;

gen catage $=1$ if agey $<1$ \& agey =.;

replace catage $=2$ if agey $>=1$ \& agey $<2$ \& agey $\sim=$.

replace catage $=3$ if agey $>=2 \&$ agey $<3 \&$ agey $\sim=$. ;

replace catage $=4$ if agey $>=3 \&$ agey $<5 \&$ agey $\sim$.

replace catage $=5$ if agey $>=5$ \& agey $<7$ \& agey $\sim=$;

replace catage $=6$ if agey $>=7$ \& agey $<10$ \& agey $\sim$.;

replace catage $=7$ if agey $>=10$ \& agey $<12$ \& agey $=$.

replace catage $=8$ if agey $>=12$ \& agey $<14$ \& agey $\sim=. ;$

replace catage $=9$ if agey $>=14$ \& agey $<16$ \& agey $\sim . ;$

replace catage $=10$ if agey $>=16$ \& agey $<18$ \& agey $=$. .

replace catage $=11$ if agey $>=18$ \& agey $<30$ \& agey $\sim=$. ,

replace catage $=12$ if agey $>=30$ \& agey $<60$ \& agey $\sim=$;

replace catage $=13$ if agey $>=60$;

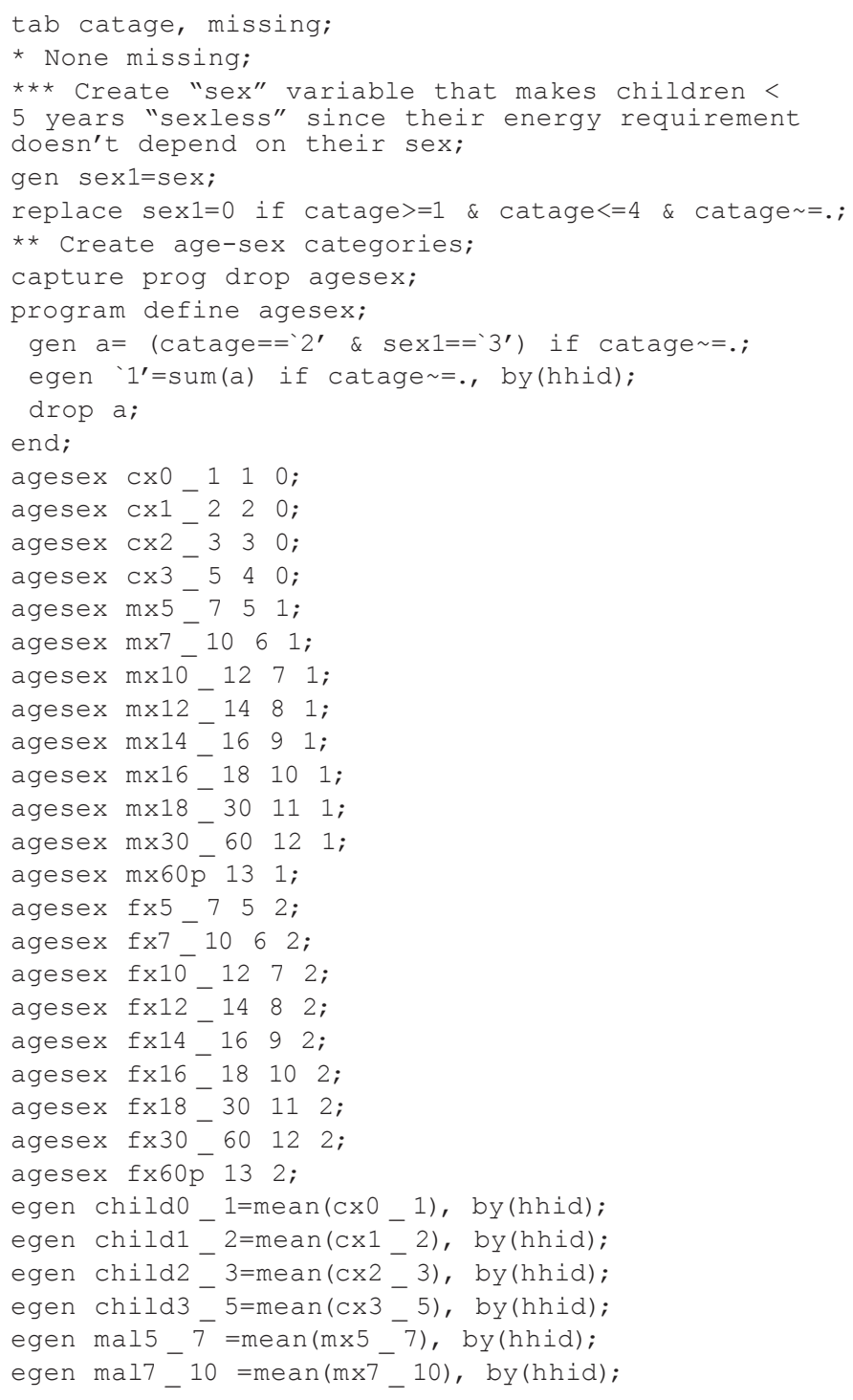


egen mal10 12=mean(mx10 12), by (hhid); egen mal12 $14=$ mean (mx12 14), by(hhid); egen mal14_16=mean(mx14_16), by(hhid); egen mal16 $18=$ mean $\left(m \times 16_{-}^{-18)}\right.$, by (hhid); egen mal18 $30=$ mean $\left(\operatorname{mx18}{ }_{-}^{-30)}\right.$, by (hhid); egen mal30_60=mean $\left(\operatorname{mx} 30 \_60\right)$, by (hhid); egen mal60p $=$ mean $(m \times 60 p)$, by (hhid); egen fem5 ${ }^{7}$ =mean(fx5 $\left.{ }^{7}\right)$, by (hhid);

egen fem7 $10=$ mean $(\mathrm{fx} 7$ 10), by(hhid). egen fem10 $12=$ mean (fx10 12), by(hhid); egen fem12 $14=$ mean(fx12 14 ), by(hhid); egen fem14 $16=$ mean $($ fx14 16$)$, by (hhid); egen fem16 $18=$ mean(fx16 ${ }_{1}$ 18), by (hhid); egen fem18 $30=$ mean $(\mathrm{fx} 18 \ldots 30)$, by (hhid); egen fem30 60=mean (fx30 60), by(hhid); egen fem60p =mean(fx60p), by(hhid): *** Collapse to the household level, collapse (mean) childo 1 child1 2 child2 3

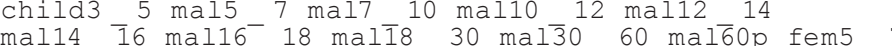
fem7 -10 fem10 12 fem12 14 fem14 $\overline{1} 6$ fem16 18

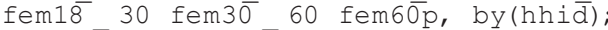

*** Calculate number of "adult equivalents", where reference category is a male $30-60$ years old (moderate activity level assumed for adults)

gen ae childo $1=820 / 2900$;

gen ae child1 2=1150/2900;

gen ae ${ }^{-}$child2 $3=1350 / 2900$

gen ae child3 5=1550/2900

gen ae ${ }_{-}$mal5 $-{ }^{-}=1850 / 2900$;

gen ae _mal7_ $10=2100 / 2900$

gen ae mal10 12=2200/2900

gen ae mal12 14=2400/2900

gen ae mal14 $16=2650 / 2900$

gen ae ${ }^{-} \operatorname{mal16}{ }^{-18} 18=2850 / 2900$;

gen ae mal18 $30=3000 / 2900$

gen ae mal30 $_{6} 60=2900 / 2900$

gen ae mal60p $=2450 / 2900$;

gen ae fem5 7 =1750/2900;

gen ae fem7_ $10=1800 / 2900$

gen ae fem10 12=1950/2900;

gen ae fem12 14=2100/2900;

gen ae fem14 $16=2150 / 2900$

gen ae ${ }_{\text {fem16 }}{ }_{\text {18 }} 2150 / 2900$;

gen ae fem18_30=2100/2900

gen ae fem30_60=2150/2900;

gen ae fem60p $=1950 / 2900$

gen hhae $=$

childo_ 1 * ae childo $1+$

childl $2 * a e^{-}$childl $-2+$

child2 ${ }_{-}^{-} *$ ae ${ }_{-}$child2 ${ }_{-} 3+$

child3 ${ }_{5}{ }^{*} \mathrm{ae}_{-}{ }^{-} \mathrm{child} 3_{-}{ }^{-}+$ mal5 ${ }_{-} 7 *$ ae ${ }_{-} \operatorname{mal5}{ }_{-} 7+$

$\operatorname{mal7}-10 * \mathrm{ae}^{-} \operatorname{mal7}-10+$

$\operatorname{mal10} 12 * \mathrm{ae}^{-} \operatorname{mal} \overline{10} 12+$

mal12 $14 *$ ae mal12 $14+$

$\operatorname{mal14}{ }_{-} 16 * a e_{-} \operatorname{mal14}{ }_{-} 16+$

mal16_18*ae_mal16_18+

mal18 $30 *$ ae mal18 $30+$

$\operatorname{mal} 3060 *$ ae $\operatorname{mal} 3060+$

mal60p * ae mal60p + fem5 7 * ae fem5 7 +

fem7 $10 *$ ae fem7 $10+$

fem $10^{-} 12 * a e^{-}$fem $10 \quad 12+$

fem12 $14 * a e$ fem12 $14+$

fem14 16 * ae fem14 $_{-} 16+$

fem16 18 * ae fem16 $18+$

fem18 30 * ae fem18 $30+$

fem30 $60 *$ ae fem30 $60+$

fem60p * ae fem60p;

label var hhae "Number of adult equivalents"

每

Part 2: Calculate household energy requirements for light activity

gen kcal req_l=

childo $1 * 820+$

child1 $2 * 1150+$

child2 $3 * 1350+$

child3 $5 * 1550+$

$\operatorname{mal5} 7 * 1850+$

$\operatorname{mal7}{ }_{-} 10 * 2100+$

mal10 $12 * 2200+$

$\operatorname{mal12} \_14 * 2400+$

mal14 $16 * 2650+$

mal18- $30 * 2600+$

$\operatorname{ma} 130-60 * 2500+$

mat $1600^{-} * 2100+$

fem5 ${ }^{7} * 1750+$

fem7 $10 * 1800+$

fem10 $12 * 1950+$

fem12 $14 * 2100+$

fem14- $16 * 2150+$

fem $16-18 * 2150+$

fem18- $30 \times 2000+$

fem60 $\mathrm{p}^{\star} 1850$;

*** Add 500 kilocalories for children <1 (lactation requirements);

replace $\mathrm{kcal}$ _req_ $1=\mathrm{kcal}$ req_ $1+500{ }^{*} \mathrm{child0} 1$;

label var kcal req 1 "H $\bar{H}$ calorie requirement for 


\section{Appendix 8}

Recommended Daily Caloric Intakes from the 2001 Expert Consultation, by Age, Sex, and Activity Level

recommended by the 2001 expert consultation on human energy requirements convened by the United Nations

University, the World Health Organization, and the Food and Agriculture Organization of the United Nations. The requirements are meant to be applied to population groups, not individuals. Those for older children, adolescents, and adults are reported for three activity levels — light, moderate, and heavy — which are defined in the consultation report (UNU, WHO, and FAO 2004), available on the Internet. It is important to note that the requirements for adults are reported for specific body weights. The accompanying table follows the convention established in the "user's digest" table of requirements that was based on the 1981 expert consultation (see Table 7): a weight for men of 65 kilograms and a weight for women of 55 kilograms is assumed. Those wishing to apply the energy requirements to a population of adults for whom specific sex-specific mean weights are known are directed to the 2001 expert consultation report.

The extra energy required by women for lactation in the first year of a child's life can be estimated from the 2001 expert consultation report as the mean of the requirement for the first six months, when breastfeeding is assumed to be exclusive (675 kilocalories), and that for the second six months, when it is partial (460 kilocalories) (UNU, WHO, and FAO 2004). The estimate is 567.5 kilocalories per day.

\begin{tabular}{lc|c}
$\begin{array}{l}\text { Age group (years) } \\
\text { Infants and } \\
\text { young children }\end{array}$ & Boys & Girls \\
$<1$ & 650 & 600 \\
\hline $1-2$ & 950 & 850 \\
\hline $2-3$ & 1,125 & 1,050 \\
\hline $3-4$ & 1,250 & 1,150 \\
\hline $4-5$ & 1,350 & 1,250 \\
\hline $5-6$ & 1,475 & 1,325
\end{tabular}




\section{Age group (years)}

Kilocalories per day

\begin{tabular}{ll|l|l}
$\begin{array}{l}\text { Older children and } \\
\text { adolescents }\end{array}$ & \multicolumn{1}{l}{$\begin{array}{l}\text { Light } \\
\text { activity }\end{array}$} & $\begin{array}{c}\text { Moderate } \\
\text { activity }\end{array}$ & $\begin{array}{c}\text { Heavy } \\
\text { activity }\end{array}$ \\
Boys & \multicolumn{3}{l}{} \\
\hline $6-7$ & 1,350 & 1,575 & 1,800 \\
\hline $7-8$ & 1,450 & 1,700 & 1,950 \\
\hline $8-9$ & 1,550 & 1,825 & 2,100 \\
\hline $9-10$ & 1,675 & 1,975 & 2,275 \\
\hline $10-11$ & 1,825 & 2,150 & 2,475 \\
\hline $11-12$ & 2,000 & 2,350 & 2,700 \\
\hline $12-13$ & 2,175 & 2,550 & 2,925 \\
\hline $13-14$ & 2,350 & 2,775 & 3,175 \\
\hline $14-15$ & 2,550 & 3,000 & 3,450 \\
\hline $15-16$ & 2,700 & 3,175 & 3,650 \\
\hline $16-17$ & 2,825 & 3,325 & 3,825 \\
\hline $17-18$ & 2,900 & 3,400 & 3,925
\end{tabular}

Age group (years)

Kilocalories per day

\begin{tabular}{ll|l|l} 
Adults & \multicolumn{1}{c}{$\begin{array}{c}\text { Light } \\
\text { activity }\end{array}$} & $\begin{array}{c}\text { Moderate } \\
\text { activity }\end{array}$ & $\begin{array}{c}\text { Heavy } \\
\text { activity }\end{array}$ \\
Men & 2,550 & 3,050 & 3,700 \\
\hline $18-30$ & 2,500 & 3,000 & 3,550 \\
\hline $30-60$ & 2,075 & 2,475 & 2,950
\end{tabular}

\section{Age group (years)}

$\begin{array}{lccc}\begin{array}{l}\text { Older children and } \\ \text { adolescents }\end{array} & \text { Light } & \text { Moderate } & \text { Heavy } \\ \text { activity } & \text { activity } & \text { activity }\end{array}$

adolescents

Girls

\begin{tabular}{ll|l|l}
\hline $6-7$ & 1,225 & 1,425 & 1,650 \\
\hline $7-8$ & 1,325 & 1,550 & 1,775 \\
\hline $8-9$ & 1,450 & 1,700 & 1,950 \\
\hline $9-10$ & 1,575 & 1,850 & 2,125 \\
\hline $10-11$ & 1,700 & 2,000 & 2,300 \\
\hline $11-12$ & 1,825 & 2,150 & 2,475 \\
\hline $12-13$ & 1,925 & 2,275 & 2,625 \\
\hline $13-14$ & 2,025 & 2,375 & 2,725 \\
\hline $14-15$ & 2,075 & 2,450 & 2,825 \\
\hline $15-16$ & 2,125 & 2,500 & 2,875 \\
\hline $16-17$ & 2,125 & 2,500 & 2,875 \\
\hline $17-18$ & 2,125 & 2,500 & 2,875
\end{tabular}

Age group (years)

Kilocalories per day

\begin{tabular}{ll|l|l} 
Adults & \multicolumn{1}{c}{$\begin{array}{c}\text { Light } \\
\text { activity }\end{array}$} & $\begin{array}{c}\text { Moderate } \\
\text { activity }\end{array}$ & $\begin{array}{c}\text { Heavy } \\
\text { activity }\end{array}$ \\
Women & \multicolumn{3}{l}{} \\
\hline $18-30$ & 2,025 & 2,375 & 2,850 \\
\hline $30-60$ & 1,980 & 2,375 & 2,850 \\
\hline$>60$ & 1,775 & 2,125 & 2,550
\end{tabular}

Source: The values for infants are the mean of the 12 monthly values reported in UNU, WHO, and FAO (2004), Table 3.2. The values for older children and adolescents are taken from Tables 4.5 and 4.6. The values for adults are derived from Tables 5.4-5.9 using the midpoint of the physical activity level value ranges given in Table 5.3 and the second interpolation method given on page 40. Notes: The values for adults assume a weight of 65 kilograms for males and a weight of 55 kilograms for females. All values are rounded to the nearest 25 kilocalories. 


\section{Appendix 9}

\section{STATA 8.0 Program for Estimating Metric Food Quantities to Replace Missing Values}

his program uses food-specific ordinary least squares regression equations to generate predicted values for each household. The independent variables are household characteristics, such as age and sex structure, education, and economic status, in addition to location.

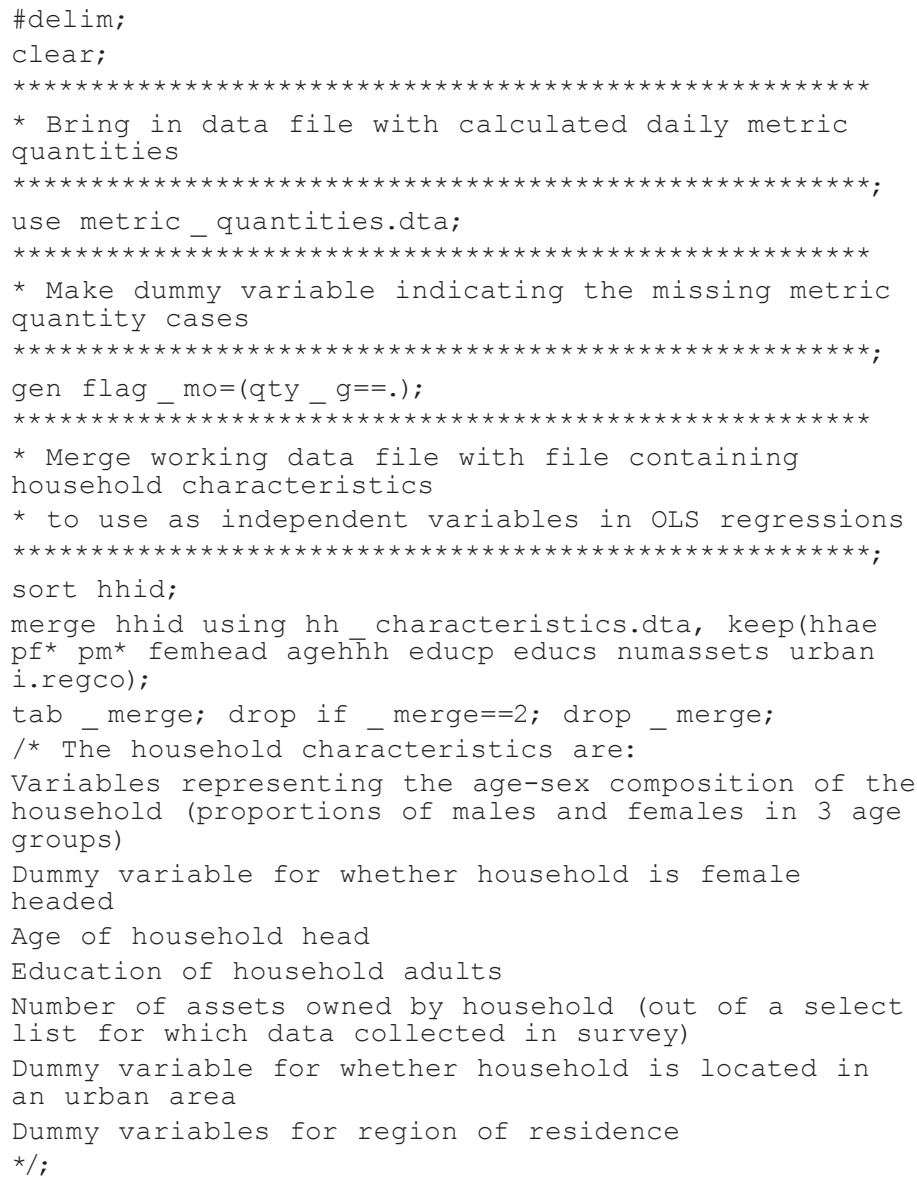


tab_merge; drop_merge;

*** Merge in foodcodes with insufficient number of observations so we can identily the ones to leave

sort foodcode

merge foodcode using racoon,

tab _merge;

tab foodcoden if merge $==3$;

drop _ merge

foodcoden | Freq. Percent Cum.

$21 \mid \begin{array}{lll}16 & 30.19 & 30.19\end{array}$

$31 \quad 1 \quad 12 \quad 22.6452 .83$

42 | $25 \quad 47.17 \quad 100.00$

Total | 53100.00

$* / ;$

*** Create a variable to hold the predicted values;

gen $p_{-}$qty $-g=. ;$

*** Run the regression program;

foreach num of numlist $1 / 20 \quad 22 / 30 \quad 32 / 41 \quad 43 / 82$

;

xi: regress qty 9 \$regvars if flag ${ }_{-} \mathrm{mo}==0$ \&

predict qty 'num',

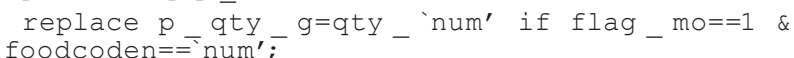

display 'num';

drop qty _ 'num';

;

*** Check to make sure all cases of flag mo==1 have a non-missing value for $\mathrm{p}-\mathrm{qty}, \mathrm{g}$ before moving on. If they don't, run regression process again with fewer

regressors;

*** If any value of $p_{-}$qty $g_{1}$ is negative, set to zero;

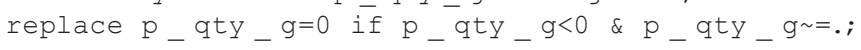

* Replace missing values with predicted values;

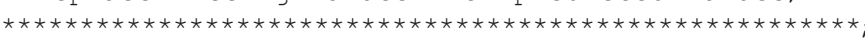

replace qty $\mathrm{g}=\mathrm{p}$ qty $\mathrm{g}$ if flag $\mathrm{mo}==1$; 


\section{Appendix 10}

\section{STATA 8.0 Program for Calculating and Cleaning Household Daily Energy Acquisition}

his program for calculating and cleaning values of household daily energy acquisition was used for the AFINS project.

\#delim;

clear;

* Bring in data file with cleaned daily metric quantities

use metric quantities cleaned.dta

* Merge in data file with energy values and edible percents.

* Match by foodcode

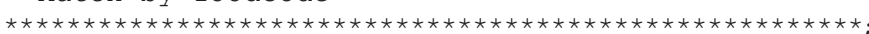

sort foodcode:

merge foodcode using calorie _ conversion factors.dta; tab merge; drop if merge $==2$; drop merge;

* Calculate the energy values of foods, after accounting

* for edible portions

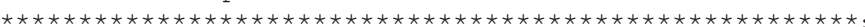

gen kcal=qty_g*(ediblpct/100)*(kcals_ $100 \mathrm{~g} / 100)$

* Calculate total daily energy acquisition for each household

collapse (sum) kcal tot=kcal flag mo, by(hhid).

* Merge working data file with file containing household size,

* household adult equivalents and household

characteristics to

* use as independent variables in OLS regressions

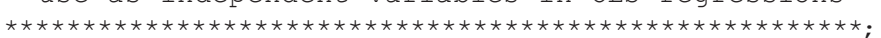
sort hhid;

merge hhid using hh characteristics.dta, keep(hhsize hhae $\mathrm{pf}^{\star} \mathrm{pm}^{\star}$ femhead agehhh educp educs numassets

tab _merge; drop if _merge==2; drop _merge;
/* The household characteristics are:

Variables representing the age-sex composition of the household

groups)

(proportions of males and females in 3 age

variable for whether household is female

headed

Age of household head

Education of household adults

Number of assets owned by household (out of a select

list for which data collected in survey)

Dummy variable for whether household is located in

an urban area

Dummy variables for region of residence

$* /$

* Calculate total daily energy acquisition per adult equivalent

gen kcal_ae=kcal_tot/hhae;

$\star$ Drop households with daily energy acquisition per adult equivalent

* greater than 12,000 kcals

drop if kcal_ae>12000\& kcal_ae =.;

* Estimate calorie availability per adult equivalent for households

* with missing values using OLS regression

xi: regress $\mathrm{kcal}$ ae hhae pf* pm* femhead agehhh educp educs numassets urban i.regco;

predict $p_{-}$kcal_ae;

$\star *$ Replace any negatives with zero;

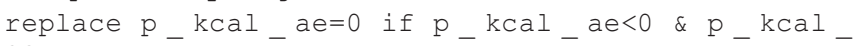
ae $=$;

* Replace missing values with predicted values;

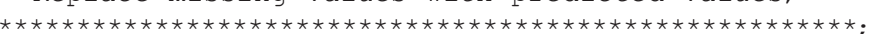
$\star *$ Replace missing with predicted;

replace kcal_ae=p_kcal_ae if $\mathrm{kcal}_{\text {_ }}$ pred =.; drop

P_kcal_ae; 
* Recalculate total household energy acquisition

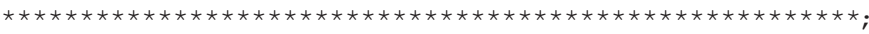
replace kcal_tot=kcal__aethhae;

* Calculate total household energy acquisition per capita

gen kcal pc=kcal tot/hhsize,

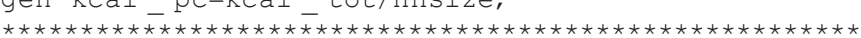

* Examine histogram of energy availability per capita

* (It should be log-Normal shaped)

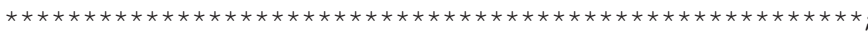

histogram kcal_pc, bin(50); 


\section{Appendix 11}

The Price-per-Calorie Method of Estimating the Energy Content of Foods Consumed outside the Home

f only total expenditures are reported for foods consumed out

of the home, the energy value of the foods can be estimated by

applying the price per calorie of foods acquired for consumption inside the home to the expenditures on food consumed outside of the home in the following steps.

1. Starting with the file containing household level values of daily energy availability from food acquired for in-home consumption (kcal_in), merge in the values of food expenditures for food acquired for in-home consumption (exp_food_in), matching by household.
2. Calculate the price per calorie of food prepared inside the home as

$$
\text { kcal_price_in = exp_food_in/kcal_in. }
$$

3. Calculate the estimated calories from food consumed outside of the home as

kcal_out $=$ exp_food_out $/ \mathrm{kcal} \_p r i c e \_$in.

In the AFINS project this calculation was only carried out if the percentage of total food expenditures on foods acquired for in-home consumption was 75 percent or greater. 


\section{References}

Alderman, H., M. Babita, G. Demombynes, N. Makhatha, and B. Ozler. 2002. How low can you go? Combining census and survey data for mapping poverty in South Africa. Journal of African Economies 11 (2): 169-200.

Arimond, M., and M. T. Ruel. 2004. Dietary diversity, dietary quality, and child nutritional status: Evidence from eleven demographic and health surveys. Report submitted to the Food and Nutrition Technical Assistance Project, Academy for Educational Development. International Food Policy Research Institute, Washington, D.C.

Benson, T., J. Kaphuka, S. Kanyanda, and R. Chinula. 2002. Malawi: An atlas of social statistics. Malawi and Washington, D.C.: National Statistical Office, Government of Malawi, and International Food Policy Research Institute.

Blair, E., and S. Burton. 1987. Cognitive processes used by survey respondents to answer behavioural frequency questions. Journal of Consumer Research 15 (3): 280-288.

Bouis, H., and L. Haddad. 1992. Are estimates of calorie income elasticities too high? A recalibration of the plausible range. Journal of Development Economics 39 (4): 333-364.

Bouis, H., and J. Hunt. 1999. Linking food and nutrition security: Past lessons and future opportunities. Asian Development Review 17 (1-2): 168-213.

Bouis, H., L. Haddad, and E. Kennedy. 1992. Does it matter how we survey demand for food? Evidence from Kenya and the Philippines. Food Policy 17 (5): 349-360.

Capéau, B., and S. Dercon. 2004. Prices, unit values and local measurement units in rural surveys: An econometric approach with an application to poverty measurement in Ethiopia. Public Economics Working Paper Series, Katholieke Universiteit Leuven, Centrum voor Economische Studien, Working Group Public Economics. Available at http://www.econ. kuleuven.ac.be/ew/academic/econover/Papers/puvlmurs.pdf. Accessed April 27, 2007.

_ 2005. Prices, unit values and local measurement units in rural surveys: An econometric approach with an application to poverty measurement in Ethiopia. Journal of African Economies 15 (2): 181-211.

COCA (Canada's Office of Consumer Affairs). 2006. The consumer trends report. Canada's Office of Consumer Affairs, Ottawa, Ontario.

Deaton, Angus. 1997. The analysis of household surveys: A microeconometric approach to development policy. Baltimore, Md.: Johns Hopkins University Press for the World Bank.

Deaton, A., and M. Grosh. 2000. Consumption. In Designing housebold survey questionnaires for developing countries: Lessons from 15 years of the Living Standards Measurement Surveys, ed. M. Grosh and P. Glewwe, 91-133. Washington, D.C.: World Bank.

Del Ninno, C., P. A. Dorosh, L. C. Smith, and D. K. Roy. 2001. The 1998 floods in Bangladesh: Disaster impacts, bousehold coping strategies, and response. IFPRI Research Report 122. Washington, D.C.: International Food Policy Research Institute.

Dignan, C. A., B. A. Burlingame, J. M. Arthur, R. J. Quigley, and G. C. Milligan. 2004. The Pacific Islands Food Composition Tables. South Pacific Commission, New Zealand Institute for Crop and Food Research Ltd. and International Network of Food Data Systems. 
Dop, M.-C., M.-C. Gomis, M. Gourdon, and S. Lesauvage. 2003. Outils d'enquête alimentaire par entretien: Élaboration au Sénégal. IRD Éditions, Collection 1, Actiques. Paris: Institut de recherche pour le développement. Economist, The. 2004. Signifying nothing? Too many economists misuse statistics. January 31,76

Eyeson, K. K., and E. K. Ankrah. 1975. Composition of foods commonly used on Ghana. Food Research Institute (CSIR) and United Nations Development Programme (UNDP) and the Food and Agriculture Organization of the United Nations (FA0). Food Research Institute, Accra, Ghana.

FAO (Food and Agriculture Organization of the United Nations). No date. Technical conversion factors for agricultural commodities. Rome. http:// www.fao.org/es/ess/pdf/tcf.pdf. Accessed August 21, 2007.

—. 1996a. The Sixth World Food Survey. Rome.

. 1996b. Rome Declaration on World Food Security and World Food Summit Plan of Action. Document prepared at the World Food Summit, Rome, November 13-17.

. 2006a. Definition and classification of commodities. Rome. http:// www.fao.org/WAICENT/faoinfo/economic/faodef/FAODEFE.HTM Accessed August 21, 2007.

- 2006b. International Food Composition Tables directory. The International Network of Food Data Systems (INFOODS). Rome. http:// www.fao.org/infoods/directory_en.stm. Accessed August 21, 2007.

—. 2006c. Nutritive factors. Statistics Division, Rome. http:/www.fao.org/ es/ess/xxx.asp. Accessed August 21, 2007

FAO, WHO, and UNU (Food and Agriculture Organization of the United Nations, World Health Organization, and United Nations University). 1985. Protein and energy requirements. Rome.

Frankenberg, E. 2000. Community and price data. In Designing bousebold survey questionnaires for developing countries: Lessons from 15 years of the Living Standards Measurement Study, ed. M. Grosh and P. Glewwe, Chapter 13. Washington, D.C.: World Bank.

Frankenberger, T., N. Mock. and P. Jere. 2005. Vulnerability assessment methodology review. Commissioned by the Southern Africa Development Community-Food, Agriculture and Natural Resources, Regional Vulnerability Assessment Committee (SADC-FANR RVAC). Tango International Inc., Tucson, Ariz., U.S.A. http://www.sahims.net/ doclibrary/Sahims_Documents/141105_RVAC_VAC_review.pdf. Accessed August 21, 2007

FSANZ (Food Standards Australia New Zealand). 2004. Explanatory notes for use with the online nutrition panel calculator (NPC). Supported by AUSNUT Special Edition (3) (Australian Food and Nutrient Database for Nutrition Labelling-Release 3). Commonwealth of Australia and FSANZ. http:// www.foodstandards.gov.au/_srcfiles/Explanatory_notes_for_the_NPC_ coldfusion_October_2004_final.pdf. Accessed April 21, 2007.

Ghana Statistical Service. No date. Consumer Price Index field sheet (price quotations of selected items). Ghana Statistical Service, Accra.

Gibson, J., and S. Rozelle. 2002. How elastic is calorie demand? Parametric, nonparametric and semiparametric results for urban Papua New Guinea. Journal of Development Studies 38 (6): 23-46.

2005. Prices and unit values in poverty measurement and tax reform analysis. World Bank Economic Review 19 (1): 69-97.

Gibson, J., J. Huang, and S. Rozelle. 2003. Improving estimates of inequality and poverty from urban China's Household Income and Expenditure survey. Review of Income and Wealth 49 (1): 53-68.

Gibson, R. S., and E. L. Ferguson. 1999. An interactive 24-hour recall for assessing the adequacy of iron and zinc intakes in developing countries. U.S. Agency for International Development and International Life Sciences Institute, Washington, D.C. http://argentina.ilsi.org/file/Recall.pdf. Accessed August 21, 2007 
Goldberger, A. 1968. The interpretation and estimation of Cobb-Douglas functions. Econometrica 35: 464-472.

Gopalan, C., B. V. Rama Sastri, and S. C. Balasubramanian. 1976. Nutritive value of Indian foods. National Institute of Nutrition, Indian Council of Medical Research, Hyderabad, India.

Graham, R., R. M. Welch, and H. E. Bouis. 2004. Addressing micronutrient malnutrition through enhancing the nutritional quality of staple foods: Principles, perspectives and knowledge gaps. Department of Plant Science, University of Adelaide, Australia; U.S. Plant, Soil and Nutrition Laboratory, USDA-ARS, Ithaca, N.Y; International Food Policy Research Institute, Washington, D.C. http://www.css.cornell.edu/FoodSystems/ AdvAgron4.html. Accessed August 21, 2007.

Grosh, M., and P. Glewwe, eds. 2000. Designing household survey questionnaires for developing countries: Lessons from 15 years of the Living Standards Measurement Surveys. Washington, D.C.: World Bank.

Grosh, M., P. Glewwe, and J. Munoz. 2000. Designing modules and assembling them into survey questionnaires. In Designing household survey questionnaires for developing countries: Lessons from 15 years of the Living Standards Measurement Surveys, ed. M. Grosh and P. Glewwe, 43-75. Washington, D.C.: World Bank.

Haddad, L., and R. Kanbur. 1990. How serious is the neglect of intrahousehold inequality? Economic Journal 100 (402): 866-881.

Hoddinott, J. 2001. Choosing outcome indicators of household food security. In Methods for rural development projects, ed. J. Hoddinott, Chapter 3. Food Security in Practice series. Washington, D.C.: International Food Policy Research Institute.

IL0 (International Labour Organization). 2004. Consumer price index man ual: Theory and practice. Geneva. http://www.ilo.org/public/english/ bureau/stat/guides/cpi/\#manual. Accessed August 21, 2007.
Ireland, J. D., and A. Moller. 2000. Review of international food classification and description. Journal of Food Composition and Analysis 13: 529-538.

Leung, W.-T. W. 1968. Food composition table for use in Africa. Food and Agriculture Organization of the United Nations, Rome, and U.S. Department of Health, Education, and Welfare, Bethesda, Md., U.S.A.

Leung, W.-T. W., R. R. Butrum, and F. H. Chang. 1972. Food composition table for use in East Asia. Food and Agriculture Organization of the United Nations, Rome, and U.S. Department of Health, Education, and Welfare, Bethesda, Md., U.S.A.

Ndaiye, A. M. 1993. Aliments Africains: Table de composition. Organisme de Recherches sur l'Alimentation et la Nutrition Africaines (ORANA), Dakar, Senegal.

NSSO Expert Group on Sampling Errors. 2003. Suitability of different reference periods for measuring household consumption: Results of a pilot study. Economic and Political Weekly 37 (4): 307-321.

Platt, B. S. 1962. Tables of representative values of foods commonly used in tropical countries. Medical Research Council, London. Reprint 1985, London School of Hygiene and Tropical Medicine, Department of Human Nutrition.

Pradhan, Menno. 2001. Welfare analysis with a proxy consumption measure: Evidence from a repeated experiment in Indonesia. CFNPP Working Paper 126. Ithaca, N.Y.: Cornell Food and Nutrition Policy Program.

Rashid, A., L. C. Smith, and T. Rahman. 2006. Determinants of dietary quality: Evidence from Bangladesh. Paper prepared for presentation at the annual meetings of the American Agricultural Economics Association, July 23-26, Long Beach, Calif., U.S.A.

Ruel, M. T. 2002. Is dietary diversity an indicator of food security or dietary quality? A review of measurement issues and research needs. Food Consumption and Nutrition Division Discussion Paper 140. Washington, D.C.: International Food Policy Research Institute. 
2003. Operationalizing diet diversity: A review of measurement issues and research priorities. Journal of Nutrition 133 (11): 3911S-3926S.

Ruel, M. T., L. Haddad, M. Arimond, D. Gilligan, N. Minot, K. Simler, and

X. Zhang. 2003. Diet quality and diet changes of the poor: A global research program to improve dietary quality, health, and nutrition. A proposal for a global research program (GRP24). International Food Policy Research Institute, Washington, D.C.

Sehmi, J. K. 1993. National food composition tables and the planning of satisfactory diets in Kenya. Ministry of Health, Government of Kenya, Nairobi.

Siong, T. E., M. I. Noor, M. N. Azudin, and K. Idris. 1997. Nutrient composition of Malaysian foods. 4th edition. Malaysian Food Composition Database Programme, c/o Institute for Medical Research, Kuala Lumpur.

Smith, L. C., and A. Subandoro. 2005. Improving the empirical basis for assessing food insecurity in developing countries: Asia. Project final report. Submitted to the Department for International Development of the United Kingdom and the Australian Agency for International Development. International Food Policy Research Institute, Washington, D.C.

Smith, L. C., H. Alderman, and D. Aduayom. 2006. Food insecurity in Sub Saharan Africa: New estimates from household expenditure surveys. IFPRI Research Report 146. Washington, D.C.: International Food Policy Research Institute.

Subramanian, S., and A. Deaton. 1996. The demand for food and calories. Journal of Political Economy 104 (1): 133-162.

Swindale, A., and P. Bilinksy. Household diet diveristy score (HDDS) for measurement of household food access: Indicator guide. 2005. Food and Nutrition Technical Assistance (FANTA) Project and Academy for Educational Development (AED), Washington, D.C.

Swindale, A., and P. Ohri-Vachaspati. 2004. Measuring housebold food consumption: A technical guide. Food and Nutrition Technical Assistance
(FANTA) Project and Academy for Educational Development (AED),

Washington, D.C.

United Nations. 1989. Housebold income and expenditure surveys: A technical study. National Household Survey Capability Programme. New York: United Nations Department of Technical Co-operation for Development Statistical Office.

UNU, WHO, and FAO (United Nations University, World Health Organization, and Food and Agriculture Organization of the United Nations). 2004. Human energy requirements: Report of a joint FAO/WHO/UNU expert consultation, Rome, October 17-24, 2001. FA0 Food and Nutrition Technical Report Series 1. Rome: FA0.

USDA (U.S. Department of Agriculture). 2003. Composition of foods, raw, processed, prepared. USDA Nutrient Database for Standard ReferenceRelease 15. Beltsville, Md., U.S.A.: USDA, Agricultural Research Service.

—. 2005. USDA National Nutrient Database for Standard Reference Release 18. USDA Agricultural Research Service, Washington, D.C. http:// www.nal.usda.gov/fnic/foodcomp/Data/SR18/sr18.html. Accessed August 21, 2007.

U.S. Department of Labor. 2006. Consumer expenditures in 2004. U.S. Bureau of Labor Statistics Report 992. Washington, D.C.

von Braun, J., M. W. Rosegrant, R. Pandaya-Lorch, M. J. Cohen, S. A. Cline,

M. A. Brown, and M. S. Bos. 2005. New risks and opportunities for food security. 2020 Discussion Paper 39, International Food Policy Research Institute, Washington, D.C.

Welch, R. 2004. Micronutrients, agriculture, and nutrition: Linkages for improved health and well being. USDA-ARS, U.S. Plant, Soil and Nutrition Laboratory, Ithaca, N.Y. http://www.css.cornell.edu/ FoodSystems/Micros\%26AgriMan1ref.html. Accessed August 21, 2007.

West, C. E., F. Pepping, and C. R. Temalilwa, eds. 1988. The composition of foods commonly eaten in East Africa. Wageningen, The Netherlands: Wageningen Agricultural University. 


\section{About the Authors}

LISA C. SMITH (1smith@chuparosa.us) is a research consultant for Technical Assistance to Non-Governmental Organizations (TANG0) in Tucson, Arizona. Her research focuses on the causes of hunger and malnutrition in developing countries and the measurement of global food security. She was formerly a research fellow in the Food Consumption and Nutrition Division of the International Food Policy Research Institute, Washington, D.C.

ALI SUBANDOR0 (asubandoro@worldbank.org) is a monitoring and evaluation consultant in the Social Development Unit of the World Bank Country Office for Indonesia. Before joining the World Bank, he was a consultant for the International Food Policy Research Institute in Washington, D.C., and the World Food Programme in Egypt, where he worked on various research and impact studies in the food and nutrition security area. Subandoro is a citizen of Indonesia.

\section{PHOTO CREDITS:}

Pages $x$ and 46, () 2003 Richard Lord

Page 4, () Trygve Bolstad / Panos Pictures

Page 12, (c) 1997 Suresh Babu / IFPRI

Page 56, (c) 1997 Neal Bliven / IFPRI

Page 74, (c) 1996 Curt Carnemark / World Bank

Page 80, (c) Jacob Silberberg / Panos Pictures 